AEI-2002-096

DAMTP-02-148

LAPTH-953/02

hep-th/0212116

\title{
Correlation Functions and Massive Kaluza-Klein Modes in the AdS/CFT Correspondence
}

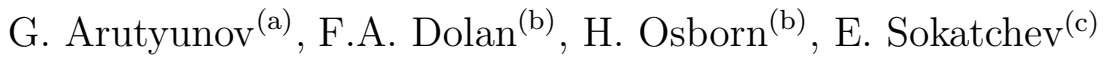 \\ (a) Max-Planck-Institut für Gravitationsphysik, Albert-Einstein-Institut, \\ Am Mühlenberg 1, D-14476 Golm, Germany \\ (b) Department of Applied Mathematics and Theoretical Physics, \\ Silver Street, Cambridge, CB3 9EW, England \\ (c) Laboratoire d'Annecy-le-Vieux de Physique Théorique ${ }^{1}$ LAPTH, B.P. 110, \\ F-74941 Annecy-le-Vieux et l'Université de Savoie
}

\begin{abstract}
We study four-point correlation functions of $\frac{1}{2}$-BPS operators in $\mathcal{N}=4 \mathrm{SYM}$ which are dual to massive KK modes in $\mathrm{AdS}_{5}$ supergravity. On the field theory side, the procedure of inserting the SYM action yields partial non-renormalisation of the four-point amplitude for such operators. In particular, if the BPS operators have dimensions equal to three or four, the corresponding four-point amplitude is determined by one or two independent functions of the two conformal cross-ratios, respectively. This restriction on the amplitude does not merely follow from the superconformal Ward identities, it also encodes dynamical information related to the structure of the gauge theory Lagrangian.

The dimension 3 BPS operator is the AdS/CFT dual of the first non-trivial massive KaluzaKlein mode of the compactified type IIB supergravity, whose interactions go beyond the level of the five-dimensional gauged $\mathcal{N}=8$ supergravity. We show that the corresponding effective Lagrangian has a surprisingly simple sigma-model-type form with at most two derivatives. We then compute the supergravity-induced four-point amplitude for the dimension 3 operators. Remarkably, this amplitude splits into a "free" and an "interacting" parts in exact agreement with the structure predicted by the insertion procedure. The underlying OPE fulfills the requirements of superconformal symmetry and unitarity.
\end{abstract}

PACS: 11.15.-q, 11.30.Pb, 11.25.Tq, 04.50.+h, 04.65.+e

Keywords: AdS/CFT, SYM theory, Supergravity.

\footnotetext{
${ }^{1}$ UMR 5108 associée à l'Université de Savoie
} 


\section{Introduction}

In the past few years the holographic relation between supergravity (string) theories on AdS backgrounds and certain conformal field theories living on the corresponding AdS boundaries has been studied and tested by various means. The most typical example is the $\mathcal{N}=4$ super-Yang-Mills (SYM) theory whose conjectured holographic dual is the type IIB supergravity (string) theory on $A d S_{5} \times S^{5}$. Our current understanding of this basic example is primarily based on superconformal kinematics, as the isometry group of the supergravity theory coincides with the superconformal symmetry group of its dual. In this respect it is highly desirable to learn how to separate the actual dynamical statements from those due to superconformal symmetry and to subsequently put the former to the test.

The string spectrum on the $A d S_{5} \times S^{5}$ background is presently unavailable. Thus, our current studies are confined to $A d S_{5}$ supergravity that is dual to the limit of the gauge theory where the 't Hooft coupling $\lambda=g_{Y M}^{2} N$ is infinite and the rank $N$ of the gauge group $\mathrm{SU}(N)$ is large. Compactifying type IIB supergravity on the five-dimensional sphere results in an infinite tower of (generically massive) Kaluza-Klein (KK) modes. According to the superconformal kinematics, their field-theory duals are the so-called $\frac{1}{2}$-BPS operators. They form short superconformal multiplets whose lowest-weight states are annihilated by half of the Poincaré supercharges. The conformal dimensions and more generally, the two- and three-point correlation functions of the $\frac{1}{2}$-BPS operators are protected from quantum corrections, but the four-point functions are not. The OPE spectrum of two $\frac{1}{2}$-BPS operators is rich, coupling-dependent and generically contains unprotected (long) supermultiplets. Thus, the four-point correlators of $\frac{1}{2}$-BPS operators encode some genuinely dynamical information and hence are interesting objects to study both in field theory and in the supergravity approximation.

The superconformal kinematics puts constraints on the four-point amplitude in the form of superconformal Ward identities. Their solution admits a functional freedom providing a window for the non-trivial dynamics. Further restrictions on the correlation functions can be obtained by a dynamical mechanism. It consists in generating the quantum corrections to the amplitude by insertion of the SYM action (the insertion procedure) and has the effect of reducing the functional freedom in the amplitude (partial non-renormalisation). This procedure is purely field-theoretic and it relies on the existence of a Lagrangian description of the theory. However, its prediction, i.e. the particular form of the amplitude compared to the general solution of the superconformal Ward identities, can be confronted with concrete supergravity-induced correlators computed in $A d S_{5}$ supergravity. This is one of the most probing tests of the AdS/CFT duality available today.

Our concrete knowledge of the supergravity-induced four-point amplitudes has up to now been exhausted by a single example, the correlator of $\frac{1}{2}$-BPS operators of the lowest allowed dimension 2. The corresponding supermultiplet is rather special, as it contains the conserved $\mathrm{R}$ symmetry current and the stress tensor of the $\mathcal{N}=4$ theory. It is dual to the graviton multiplet of the gauged $\mathcal{N}=8$ five-dimensional supergravity comprising the massless KK modes of the type IIB supergravity compactification.

Clearly, to start bringing out the flavor of the more involved ten-dimensional physics one has to go beyond the massless sector of the theory and obtain new examples of supergravityinduced four-point correlators involving BPS operators of higher dimension. In this paper we make a first step in this direction by studying the general form of the four-point amplitude 
for $\frac{1}{2}$-BPS operators of dimension 3 and then comparing it to an explicit supergravity computation.

As mentioned earlier, this time we cannot restrict ourselves to the gauged $\mathcal{N}=8$ supergravity but should rather start from the full ten-dimensional theory. The $\frac{1}{2}$-BPS operators of dimension $k$ are AdS/CFT dual to the KK modes $s_{k}$ transforming in the irrep $[0, k, 0]$ of $\mathrm{SO}(6)$. To compute the corresponding supergravity-induced four-point amplitude one first has to find the action for the fields $s_{k}$ up to quartic order. This is a hard problem primarily due to the absence of a suitable Lagrangian formulation of the type IIB supergravity (the well-known self-duality problem). One way to solve it is to expand the covariant equations of motion of type IIB supergravity around the background solution and find the quadratic and cubic corrections to the free AdS equations of motion. Using the freedom of perturbative field redefinitions, one is able to recast these equations into a Lagrangian form. This approach has lead to an effective action in $A d S_{5}$ space which allows the computation of fourpoint correlators for $\frac{1}{2}$-BPS operators of arbitrary dimension (see Section 4 for references and a brief review). The effective action has the following remarkable properties:

- the quartic couplings contain terms with four and two derivatives only;

- it admits a consistent truncation to the massless graviton multiplet and the corresponding action coincides with that of the gauged $\mathcal{N}=8$ five-dimensional supergravity on $A d S_{5}$;

- the quartic couplings corresponding to the so-called extremal or subextremal correlators vanish;

- the four-point correlation functions in the boundary CFT derived from this action obey the predictions of the field-theoretic insertion procedure.

The latter property is probably the most non-trivial one. It has so far been verified for $\frac{1}{2}$-BPS operators of dimension 2. One of the principal aims of the present paper is to carry out a similar test in the case of dimension 3. Again we find a remarkable agreement. One of the surprising features of the supergravity-induced amplitude in both cases is the splitting into a "free" and an "interacting" parts, exactly following the field theory pattern where the free amplitude is supposed to receive quantum corrections. This is indeed unexpected, since in supergravity there is no analog of the coupling constant which makes the splitting natural in field theory. All this is not only a non-trivial check of the effective supergravity action but it also supports the field-theoretic arguments for the partially non-renormalised form of the amplitude. Last but not least, it provides strong evidence for the AdS/CFT duality.

It should also be pointed out that after specifying the explicit representation content of the fields involved, the resulting quartic effective action turns out to be rather simple. The quartic couplings with four-derivative vertices actually vanish, at least in the case we consider here. In other words, the action is of the sigma-model type. Therefore it would be highly desirable to develop a systematic superspace procedure for the construction of the supergravity effective action. This could eventually unravel its hidden symmetry structure and simplicity.

Another interesting problem is to understand the relationship between four-point amplitudes of $\frac{1}{2}$-BPS operators of different dimensions. Indeed, adding at least one KK mode 
to the massless graviton multiplet causes the whole infinite tower of massive KK modes to emerge. In other words, all the higher KK modes are tightly bound together in a unique interacting Lagrangian. It seems plausible that this would have implications for the correlators derived from such a Lagrangian.

Finally, one can reverse the logic of passing from supergravity to gauge theory and ask the question to what extent are the quartic (contact) terms in the effective action (or even the whole action) fixed by requiring the corresponding four-point amplitude to satisfy the restrictions imposed by the insertion procedure. We hope to come back to this question in the future.

The paper is organised as follows. In Section 2 we discuss the general form of the fourpoint amplitude of $\frac{1}{2}$-BPS operators based on the conformal, $R$ and crossing symmetries. In Section 3 we provide field-theoretic arguments leading to the partially non-renormalised form of the amplitude. We show that for the "quantum part" of the four-point correlator of dimension 3 operators the insertion formula predicts a single function of the conformal cross-ratios instead of three such functions. In Section 4 we compute the corresponding supergravity-induced four-point amplitude and in Section 5 we verify the CFT predictions from Section 3. Finally, in Section 6 we perform a partial OPE analysis of the amplitude and obtain the large $N$ corrections to the scaling dimensions of the long multiplets. The computational details are gathered in several appendices.

\section{General four-point amplitudes}

According to the AdS/CFT duality conjecture [1, 2, 3, the KK modes of the $A d S_{5} \times S^{5}$ compactification of type-IIB supergravity are mapped into $\frac{1}{2}$-BPS multiplets of the $\mathcal{N}=4$ SYM theory whose lowest components are scalar fields $\mathcal{O}^{I}$ of conformal dimension $k \geq 2$ in the irrep $[0, k, 0]$ of the $R$ symmetry group $\mathrm{SO}(6) \sim \mathrm{SU}(4)$. For $k=2$ this is the so-called stress-tensor multiplet corresponding to the massless AdS supergravity multiplet. For $k \geq 3$ we are dealing with non-trivial (massive) KK modes. In this section we discuss the general structure of the correlator of four identical operators $\mathcal{O}^{I}$, predicted on the basis of conformal, $R$ and crossing symmetry. In particular, we determine the number of independent conformal invariant functions which specify such correlators for $k=2,3,4$ and we outline a method to do this for general $k$.

The composite operators $\mathcal{O}^{I}$ with a suitably normalised two-point function are of the form

$$
\mathcal{O}^{I}=C_{i_{1} \cdots i_{k}}^{I} \operatorname{Tr}\left(\phi^{i_{1}} \cdots \phi^{i_{k}}\right) .
$$

Here $\phi^{i}, i=1, \ldots, 6$ are the $\mathcal{N}=4$ SYM scalars and $C_{i_{1} \ldots i_{k}}^{I}$ are traceless symmetric tensors obeying the normalisation condition $C_{i_{1} \cdots i_{k}}^{I} C_{i_{1} \cdots i_{k}}^{J}=\delta^{I J}$, which describe the irreducible representations $[0, k, 0]$.

The $R$ symmetry content of the OPE of two identical operators $\mathcal{O}^{I_{1}}$ and $\mathcal{O}^{I_{2}}$ is determined by the corresponding tensor product decomposition

$$
[0, k, 0] \times[0, k, 0]=\sum_{p=0}^{k} \sum_{q=0}^{k-p}[q, 2 k-2 q-2 p, q] .
$$


The total number of terms in the right-hand side of eq.(2.2) is $\frac{1}{2}(k+1)(k+2)$. According to the general arguments in [4-[7], all the OPE channels with $q=0,1$ contain only protected (BPS or semishort) operators.

The decomposition (2.2) provides the basis for an OPE expansion of the four-point amplitude $\langle\mathcal{O}(1) \mathcal{O}(2) \mathcal{O}(3) \mathcal{O}(4)\rangle$. However, in field theory there exists a different basis which naturally arises by connecting the four points with free propagators (Wick contractions). The free propagator for the elementary $\mathcal{N}=4$ SYM scalars is

$$
\left\langle\phi^{i}\left(x_{1}\right) \phi^{j}\left(x_{2}\right)\right\rangle=\frac{\delta^{i j}}{x_{12}^{2}} .
$$

A convenient way of keeping track of the $\mathrm{SO}(6)$ indices is to project them with "harmonic variables", i.e. a complex vector $u_{i}$ satisfying the conditions

$$
u_{i} u_{i}=0, \quad u_{i} \bar{u}_{i}=1
$$

This vector provides a harmonic description of the coset space $\mathrm{SO}(6) / \mathrm{SO}(2) \times \mathrm{SO}(4)$. Indeed, the vector $u_{i}$ subject to the constraints (2.4) and modulo $\mathrm{U}(1) \sim \mathrm{SO}(2)$ phase transformations contains exactly the eight real coordinates of the manifold. With its help we can project the operator $\mathcal{O}^{I}$ onto the highest-weight state of the representation $[0, k, 0]$ :

$$
\mathcal{O}^{(k)}=u_{i_{1}} \cdots u_{i_{k}} \operatorname{Tr}\left(\phi^{i_{1}} \cdots \phi^{i_{k}}\right) .
$$

Here the Dynkin label $k$ is identified with the $\mathrm{U}(1)$ charge of the projection (2.5) (assuming that the vector $u_{i}$ carries $\mathrm{U}(1)$ charge +1$)$. Further, using two copies of the harmonic variables, one for each point, we can project the propagator (2.3):

$$
\langle\phi(1) \phi(2)\rangle=\frac{u_{1}^{i} u_{2}^{i}}{x_{12}^{2}} \equiv \frac{(12)}{x_{12}^{2}}=\frac{(21)}{x_{21}^{2}} .
$$

Now we can start constructing four-point functions by connecting pairs of points by the propagators (2.6). The simplest case corresponds to $k=1$, i.e., to only one propagator leaving or entering each point. In this way we find the three basic propagator structures depicted in Figure 1:

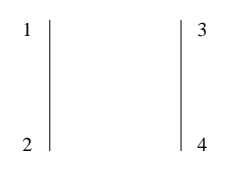

A

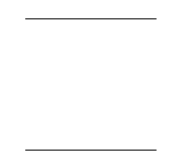

B

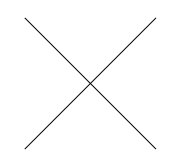

C

Figure 1: Basic contractions

The corresponding expressions involving four sets of space-time and harmonic variables are:

$$
A=\frac{(12)(34)}{x_{12}^{2} x_{34}^{2}}, \quad B=\frac{(14)(23)}{x_{14}^{2} x_{23}^{2}}, \quad C=\frac{(13)(24)}{x_{13}^{2} x_{24}^{2}} .
$$


Symbolically, the propagator basis for a four-point amplitude with arbitrary $k$ can be obtained by the following "binomial expansion"

$$
(A+B+C)^{k} \rightarrow \sum A^{m} B^{n} C^{l} .
$$

Here the sum goes over the integers $(m, n, l)$ such that $m \geq n \geq l \geq 0$ and $m+n+l=k$. For a given set $(m, n, l)^{1}$ the permutations of $A, B, C$ correspond to all possible graphs in the equivalence class obtained by crossing symmetry. It is then clear that the number of such permutations is equal to six if $m \neq n \neq l$, or to three if any two of the labels coincide but are different from the third, and to one if $m=n=l$. For example, in the case $k=2$ we obtain six graphs combined into two crossing-equivalence classes $(2,0,0)$ and $(1,1,0)$ and depicted in Figure 2.
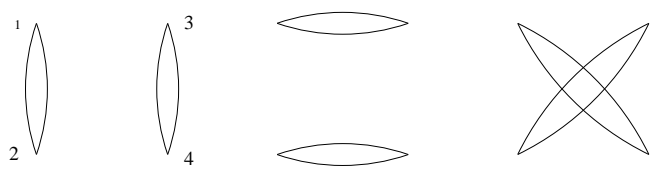

$(2,0,0)$
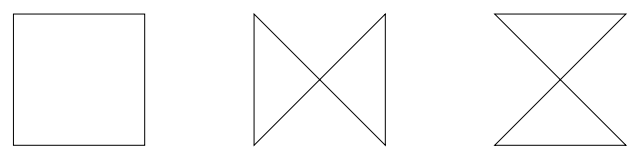

$(1,1,0)$

Figure 2: The case $k=2$

The expressions of, e.g., the first graphs in each line in Figure 2 read:

$$
\begin{aligned}
& A A=\frac{(12)^{2}(34)^{2}}{x_{12}^{4} x_{34}^{4}}=1_{i_{1}} 1_{j_{1}} 2_{i_{2}} 2_{j_{2}} 3_{i_{3}} 3_{j_{3}} 4_{i_{4}} 4_{j_{4}} \frac{\delta_{i_{2}}^{\left\{i_{1}\right.} \delta_{j_{2}}^{\left.j_{1}\right\}} \delta_{i_{4}}^{\left\{i_{3}\right.} \delta_{j_{4}}^{\left.j_{3}\right\}}}{x_{12}^{4} x_{34}^{4}}, \\
& A B=\frac{(12)(23)(34)(41)}{x_{12}^{2} x_{23}^{2} x_{34}^{2} x_{41}^{2}}=1_{i_{1}} 1_{j_{1}} 2_{i_{2}} 2_{j_{2}} 3_{i_{3}} 3_{j_{3}} 4_{i_{4}} 4_{j_{4}} \frac{\delta_{\left\{i_{2}\right.}^{\left\{i_{1}\right.} \delta_{\left\{i_{4}\right.}^{\left.j_{1}\right\}} \delta_{\left.j_{4}\right\}}^{\left\{i_{3}\right.} \delta_{\left.j_{4}\right\}}^{\left.j_{3}\right\}}}{x_{12}^{2} x_{23}^{2} x_{34}^{2} x_{41}^{2}}
\end{aligned}
$$

where $n_{i}$ is a shorthand for the harmonic $u_{n}^{i}$ at point $n$. Note that the traceless symmetrisation denoted by \{\} is automatic, given the fact that the harmonic variables commute and satisfy the defining conditions (2.4). Since the harmonics at each point are independent variables, we can remove them and thus obtain the explicit tensor structure made out of Kronecker deltas.

Each of the six propagator structures in Figure 2 can be multiplied by an arbitrary function of the conformal invariant cross-ratios

$$
s=\frac{x_{12}^{2} x_{34}^{2}}{x_{13}^{2} x_{24}^{2}}, \quad t=\frac{x_{14}^{2} x_{23}^{2}}{x_{13}^{2} x_{24}^{2}} .
$$

In this way we obtain the most general four-point amplitude for operators $\mathcal{O}^{(2)}$ with the required $\mathrm{SO}(6)$ and conformal transformation properties:

$\left\langle\mathcal{O}^{(2)}\left(x_{1}\right) \mathcal{O}^{(2)}\left(x_{2}\right) \mathcal{O}^{(2)}\left(x_{3}\right) \mathcal{O}^{(2)}\left(x_{4}\right)\right\rangle$

\footnotetext{
${ }^{1}$ The labels $(m, n, l)$ should not be confused with the Dynkin labels $[m, n, l]$.
} 


$$
\begin{aligned}
= & a_{1}(s, t) \frac{(12)^{2}(34)^{2}}{x_{12}^{4} x_{34}^{4}}+a_{2}(s, t) \frac{(13)^{2}(24)^{2}}{x_{13}^{4} x_{24}^{4}}+a_{3}(s, t) \frac{(14)^{2}(23)^{2}}{x_{14}^{4} x_{23}^{4}} \\
& +b_{1}(s, t) \frac{(13)(14)(23)(24)}{x_{13}^{2} x_{14}^{2} x_{23}^{2} x_{24}^{2}}+b_{2}(s, t) \frac{(12)(14)(23)(34)}{x_{12}^{2} x_{14}^{2} x_{23}^{2} x_{34}^{2}}+b_{3}(s, t) \frac{(12)(13)(24)(34)}{x_{12}^{2} x_{13}^{2} x_{24}^{2} x_{34}^{2}}
\end{aligned}
$$

We still need to impose the full crossing symmetry of this correlator. This is very easy to do, since the crossing properties of the structures in Figure 2 are obvious. Thus, the three coefficients $a_{i}$ in (2.10) correspond to the crossing class $(2,0,0)$ and transform into each other:

$$
\begin{aligned}
& a_{1}(s, t)=a_{3}(t, s)=a_{1}(s / t, 1 / t) \\
& a_{2}(s, t)=a_{2}(t, s)=a_{3}(s / t, 1 / t) ;
\end{aligned}
$$

the same applies to the coefficients $b_{i}$ from the class $(1,1,0)$ :

$$
\begin{aligned}
& b_{1}(s, t)=b_{3}(t, s)=b_{1}(s / t, 1 / t) \\
& b_{2}(s, t)=b_{2}(t, s)=b_{3}(s / t, 1 / t) .
\end{aligned}
$$

Thus, the crossing invariant correlator for $\mathcal{O}^{(2)}$ is in general determined by two independent functions, one of the $a_{i}$ 's and one of the $b_{i}$ 's.

Having explained the case $k=2$ in detail, we can immediately generalize to the main case of interest in this paper, $k=3$ (or to any higher value of $k$ ). The decomposition of the tensor product (2.2) reads

$$
\begin{aligned}
{[0,3,0]_{50} \times[0,3,0]_{50}=} & {[0,0,0]_{1}+[0,2,0]_{20}+[0,4,0]_{105} } \\
& +[0,6,0]_{336}+[2,0,2]_{84}+[2,2,2]_{729} \\
& +[1,0,1]_{15}+[1,2,1]_{175}+[3,0,3]_{300}+[1,4,1]_{735} .
\end{aligned}
$$

The subscripts indicate the dimension of the corresponding irrep. The irreps in the first two lines of eq.(2.13) are symmetric and those in the third line are antisymmetric in the indices $I_{1}, I_{2}$. Thus, the corresponding OPE will have ten different $\mathrm{SO}(6)$ channels. Note that among them only three may contain unprotected operators: $[0,0,0],[0,2,0]$ and $[1,0,1]$.

In the propagator basis the ten structures are organised in three crossing-equivalence classes, $(3,0,0),(2,1,0)$ and $(1,1,1)$, depicted in Figure 3. The most general $\mathrm{SO}(6)$ and conformally covariant four-point function for the operators $\mathcal{O}^{(3)}$ is of the form

$$
\begin{aligned}
\left\langle\mathcal{O}^{(3)}\right. & \left.\left(x_{1}\right) \mathcal{O}^{(3)}\left(x_{2}\right) \mathcal{O}^{(3)}\left(x_{3}\right) \mathcal{O}^{(3)}\left(x_{4}\right)\right\rangle \\
= & a_{1}(s, t) \frac{(12)^{3}(34)^{3}}{x_{12}^{6} x_{34}^{6}}+a_{2}(s, t) \frac{(13)^{3}(24)^{3}}{x_{13}^{6} x_{24}^{6}}+a_{3}(s, t) \frac{(14)^{3}(23)^{3}}{x_{14}^{6} x_{23}^{6}} \\
& +b_{1}(s, t) \frac{(12)^{2}(34)^{2}(13)(24)}{x_{12}^{4} x_{34}^{4} x_{13}^{2} x_{24}^{2}}+b_{2}(s, t) \frac{(12)^{2}(34)^{2}(14)(23)}{x_{12}^{4} x_{34}^{4} x_{14}^{2} x_{23}^{2}} \\
& +b_{3}(s, t) \frac{(14)^{2}(23)^{2}(13)(24)}{x_{14}^{4} x_{23}^{4} x_{13}^{2} x_{24}^{2}}+b_{4}(s, t) \frac{(14)^{2}(23)^{2}(12)(34)}{x_{14}^{4} x_{23}^{4} x_{12}^{2} x_{34}^{2}} \\
& +b_{5}(s, t) \frac{(13)^{2}(24)^{2}(12)(34)}{x_{13}^{4} x_{24}^{4} x_{12}^{2} x_{34}^{2}}+b_{6}(s, t) \frac{(13)^{2}(24)^{2}(14)(23)}{x_{13}^{4} x_{24}^{4} x_{14}^{2} x_{23}^{2}} \\
& +c(s, t) \frac{(12)(13)(14)(23)(24)(34)}{x_{12}^{2} x_{13}^{2} x_{14}^{2} x_{23}^{2} x_{24}^{2} x_{34}^{2}} .
\end{aligned}
$$



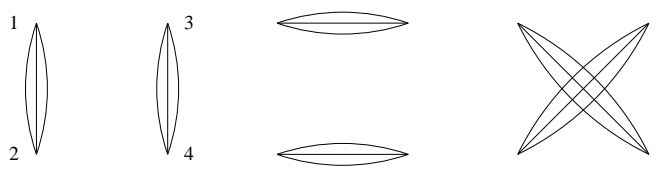

$(3,0,0)$
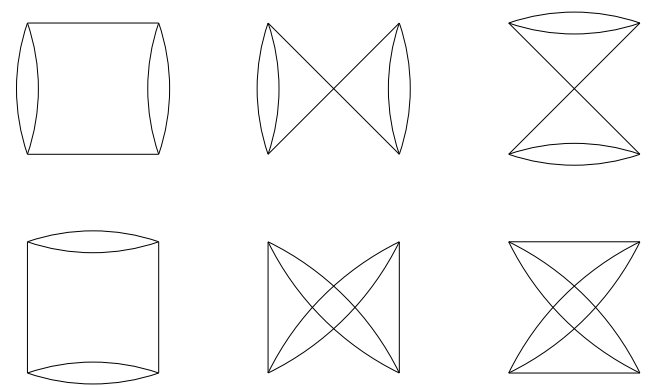

$(2,1,0)$

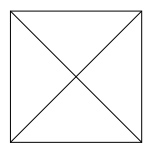

$(1,1,1)$

Figure 3: The case $k=3$

Crossing symmetry imposes relations among the functions $a_{i}(s, t), b_{i}(s, t)$. For the coefficients $a_{i}$ they are the same as in (2.11), for the coefficients $b_{i}$ they are:

$$
\begin{array}{ll}
b_{2}(s, t)=b_{1}(s / t, 1 / t), & b_{3}(s, t)=b_{1}(t, s), \quad b_{4}(s, t)=b_{1}(t / s, 1 / s) \\
b_{5}(s, t)=b_{1}(1 / s, t / s), & b_{6}(s, t)=b_{1}(1 / t, s / t)
\end{array}
$$

finally, the coefficient $c(s, t)$ must obey the symmetry conditions

$$
c(s, t)=c(t, s)=c(s / t, 1 / t)
$$

Thus, after taking crossing symmetry into account we have three independent coefficient functions, for instance, $a_{1}(s, t), b_{1}(s, t)$ and $c(s, t)$, one for each equivalence class in Figure 3. They can be split into a free field theory contribution and a quantum correction part. In free field theory the coefficient functions for the canonically normalised operators are given by $\left(b_{i}\right.$ and $c$ have been calculated in the large $N$ limit)

$$
a_{i}=1, \quad b_{i}=\frac{9}{N^{2}}, \quad c=\frac{18}{N^{2}} .
$$

As will be shown in the next section, the dynamics of the $\mathcal{N}=4$ SYM theory encoded in the insertion formula implies further non-trivial algebraic relations among the "quantum parts" of these coefficient functions leaving a single function of the conformal cross-ratios, e.g. $a_{2}(s, t)$, as the only undetermined functional freedom. 
The method developed in this section and explicitly applied to $k=2,3$ can easily be generalised to any value of $k .^{2}$ As explained earlier, the number of crossing-equivalence classes and thus of independent functions in the amplitude corresponds to the possible splittings $(m, n, l)$ of $k$. We mention that for $k=4$ there are four such splittings: $(4,0,0)$, $(3,1,0),(2,2,0)$ and $(2,1,1)$ with multiplicities $3,6,3$ and 3 , respectively.

Concluding this section we point out that the discussion of AdS supergravity in Section 4 requires a different set of harmonic variables, this time on the coset space $\mathrm{SO}(6) / \mathrm{SO}(5) \sim$ $S^{5}$. In contrast to the four-dimensional complex manifold $\mathrm{SO}(6) / \mathrm{SO}(2) \times \mathrm{SO}(4)$ considered in this section, $S^{5}$ is a real five-dimensional manifold. Thus, we are forced to describe the same representations of $\mathrm{SO}(6) \sim \mathrm{SU}(4)$ in terms of the complex harmonics $u_{i}$ in the context of CFT and in terms of the real ones on $S^{5}$ in the context of AdS supergravity. The transition from one description to the other is not direct, we need to exhibit the index structure of the representations. To this end we can use the tensor $C^{I}$ defined in (2.1). Take, for instance, the case $k=2$ and consider the four-point block $A A$ (2.9). Removing the harmonics and then converting the pairs of vector indices $i j$ into indices $I$ of the irrep 20, we obtain

$$
(A A)^{I_{1} I_{2} I_{3} I_{4}}=C_{i_{1} j_{1}}^{I_{1}} C_{i_{2} j_{2}}^{I_{2}} C_{i_{3} j_{3}}^{I_{3}} C_{i_{4} j_{4}}^{I_{4}} \frac{\delta_{i_{2}}^{\left\{i_{1}\right.} \delta_{j_{2}}^{\left.j_{1}\right\}} \delta_{i_{4}}^{\left\{i_{3}\right.} \delta_{j_{4}}^{\left.j_{3}\right\}}}{x_{12}^{4} x_{34}^{4}} \equiv \frac{\delta^{12} \delta^{34}}{x_{12}^{4} x_{34}^{4}} .
$$

In what follows we will systematically use a compact notation for the tensor structures where the indices $I_{1}, I_{2}, I_{3}, I_{4}$ will be replaced by 1234. In (2.18) the tensor is just the identity $\delta^{12} \delta^{34}$. For the block $A B$ we find

$$
(A B)^{I_{1} I_{2} I_{3} I_{4}}=C_{i_{1} j_{1}}^{I_{1}} C_{i_{2} j_{2}}^{I_{2}} C_{i_{3} j_{3}}^{I_{3}} C_{i_{4} j_{4}}^{I_{4}} \frac{\delta_{\left\{i_{2}\right.}^{\left\{i_{1}\right.} \delta_{\left\{i_{4}\right.}^{\left.j_{1}\right\}} \delta_{\left.j_{4}\right\}}^{\left\{i_{3}\right.} \delta_{\left.j_{2}\right\}}^{\left.j_{3}\right\}}}{x_{12}^{2} x_{23}^{2} x_{34}^{2} x_{41}^{2}} \equiv \frac{T^{1234}}{x_{12}^{2} x_{23}^{2} x_{34}^{2} x_{41}^{2}} .
$$

For $k=3$ we need three types of tensors:

$$
\begin{aligned}
\delta^{12} \delta^{34} & =C_{i j k}^{I_{1}} C_{i j k}^{I_{2}} C_{m l n}^{I_{3}} C_{m l n}^{I_{4}}, \\
C^{1234} & =C_{i j k}^{I_{1}} C_{i j s}^{I_{2}} C_{m l k}^{I_{3}} C_{m l s}^{I_{4}}, \\
S^{1234} & =C_{i j k}^{I_{1}} C_{i m n}^{I_{2}} C_{j m s}^{I_{3}} C_{k n s}^{I_{4}},
\end{aligned}
$$

and their permutations. The tensor $S^{1234}$ is totally symmetric, while $C^{1234}$ obeys the following symmetry relations

$$
C^{1234}=C^{2143}=C^{3412}=C^{4321} .
$$

In this notation eq.(2.14) becomes

$$
\begin{aligned}
& \left\langle\mathcal{O}^{1}\left(x_{1}\right) \mathcal{O}^{2}\left(x_{2}\right) \mathcal{O}^{3}\left(x_{3}\right) \mathcal{O}^{4}\left(x_{4}\right)\right\rangle \\
& =a_{1}(s, t) \frac{\delta^{12} \delta^{34}}{x_{12}^{6} x_{34}^{6}}+a_{2}(s, t) \frac{\delta^{13} \delta^{24}}{x_{13}^{6} x_{24}^{6}}+a_{3}(s, t) \frac{\delta^{14} \delta^{23}}{x_{14}^{6} x_{23}^{6}} \\
& \quad+b_{1}(s, t) \frac{C^{1234}}{x_{12}^{4} x_{34}^{4} x_{13}^{2} x_{24}^{2}}+b_{2}(s, t) \frac{C^{1243}}{x_{12}^{4} x_{34}^{4} x_{14}^{2} x_{23}^{2}}+b_{3}(s, t) \frac{C^{3214}}{x_{23}^{4} x_{14}^{4} x_{13}^{2} x_{24}^{2}}
\end{aligned}
$$

\footnotetext{
${ }^{2} \mathrm{~A}$ different counting method as well as a general formula for the number of independent functions has been given in $[8]$.
} 


$$
\begin{aligned}
& +b_{4}(s, t) \frac{C^{3241}}{x_{23}^{4} x_{14}^{4} x_{34}^{2} x_{12}^{2}}+b_{5}(s, t) \frac{C^{4231}}{x_{24}^{4} x_{13}^{4} x_{12}^{2} x_{34}^{2}}+b_{6}(s, t) \frac{C^{4213}}{x_{24}^{4} x_{13}^{4} x_{14}^{2} x_{23}^{2}} \\
& +c(s, t) \frac{S^{1234}}{x_{12}^{2} x_{13}^{2} x_{14}^{2} x_{23}^{2} x_{24}^{2} x_{34}^{2}}
\end{aligned}
$$

\section{The insertion formula and partial non-renormalisation}

Further, dynamical information about the four-point correlators can be obtained by using a well-known quantum field theory procedure, the insertion of the action as an extra fifth point. ${ }^{3}$ Let us consider the correlator

$$
\left\langle\mathcal{O}^{\left(k_{1}\right)} \mathcal{O}^{\left(k_{2}\right)} \mathcal{O}^{\left(k_{3}\right)} \mathcal{O}^{\left(k_{4}\right)}\right\rangle
$$

of four, a priori different, $\frac{1}{2}$-BPS operators $\mathcal{O}^{\left(k_{i}\right)}$ in the $\mathrm{SO}(6)$ irreps $\left[0, k_{i}, 0\right]$. A particular case is obtained by setting $k=2$, and this is the so-called stress-tensor multiplet. Its $\theta$ expansion contains the on-shell $\mathcal{N}=4 \mathrm{SYM}$ Lagrangian at the level $(\theta)^{4}$. Now, the derivative of the correlator (3.1) with respect to the YM coupling $g^{2}$ can be represented in the form

$$
\frac{\partial}{\partial g^{2}}\left\langle\mathcal{O}^{\left(k_{1}\right)} \mathcal{O}^{\left(k_{2}\right)} \mathcal{O}^{\left(k_{3}\right)} \mathcal{O}^{\left(k_{4}\right)}\right\rangle \propto \int \mathrm{d}^{4} x_{0} \mathrm{~d}^{4} \theta_{0}\left\langle\mathcal{O}^{(2)}(0) \mathcal{O}^{\left(k_{1}\right)} \mathcal{O}^{\left(k_{2}\right)} \mathcal{O}^{\left(k_{3}\right)} \mathcal{O}^{\left(k_{4}\right)}\right\rangle
$$

The integration over the insertion point 0 with a specially chosen superspace measure corresponds to a "superaction" in the terminology of Ref. [10]. In principle, the smallest invariant subspace of $\mathcal{N}=4$ superspace, suitable for describing $\frac{1}{2}$-BPS multiplets, involves $8=16 / 2 \theta$ 's, while the integration in (3.2) goes over only four $\theta$ 's. The point however is that the operator $\mathcal{O}^{(2)}$ is not just $\frac{1}{2}$-BPS short but it is even "ultrashort" in the terminology of [11]. This means that its $\theta$ expansion effectively terminates at four $\theta$ 's, all the higherorder terms being total space-time derivatives of the lower terms. This explains why the integration in (3.2) is supersymmetric. Note also that the measure $d^{4} \theta_{0}$ in (3.2) involves only chiral $\theta$ 's (left-handed, or right-handed in the conjugate form).

Using the $\mathcal{N}=4$ version [12] of harmonic superspace [13], one can show [14, 15, 16, 8] that the five-point correlator $\left\langle\mathcal{O}^{(2)}(0) \mathcal{O}^{\left(k_{1}\right)} \mathcal{O}^{\left(k_{2}\right)} \mathcal{O}^{\left(k_{3}\right)} \mathcal{O}^{\left(k_{4}\right)}\right\rangle$ gives rise to a nilpotent superconformal covariant. Nilpotent covariants for $\frac{1}{2}$-BPS operators do not exist if the number of points $n \leq 4$. Indeed, the $\frac{1}{2}$-BPS condition tells us that at each point such a covariant should depend on (a particularly chosen, or harmonic-projected) half of the $\theta$ 's. Since this object must be covariant under two full supersymmetries (Poincaré and special conformal), for $n \leq 4$ there exist no invariant combinations of the $\theta$ 's and hence no way to form nilpotent covariants. However, starting with $n=5$ this becomes possible. On the other hand, since the $\theta$ measure in (3.2) is chiral, the $\theta$ expansion of the five-point correlator must start with four left-handed $\theta$ 's. So, it must be precisely of the nilpotent type.

Although it is in principle known how to construct such nilpotent covariants [14, the explicit expression in $\mathcal{N}=4$ harmonic superspace is rather complicated and is currently not available. Instead, it is much easier to carry out the insertion procedure in $\mathcal{N}=2$ harmonic superspace. The idea is to project the $\mathcal{N}=4$ composite operators $\mathcal{O}^{\left(k_{i}\right)}$ on $\mathcal{N}=2$. In particular, one finds $\mathcal{N}=2$ projections which involve only hypermultiplets. All

\footnotetext{
${ }^{3}$ In the context of $\mathcal{N}=4 \mathrm{SYM}$ theory this procedure was first considered in 9 .
} 
the coefficient functions of the initial $\mathcal{N}=4$ correlator can be read off from a few such hypermultiplet projections. The advantage of this $\mathcal{N}=2$ approach is that the explicit form of the corresponding five-point nilpotent covariants is much simpler. A further, and even more important feature of the $\mathcal{N}=2$ formalism, is the possibility to formulate both ingredients of the $\mathcal{N}=4$ theory ( $\mathcal{N}=2 \mathrm{SYM}$ and hypermultiplets) off shell and to develop a straightforward quantisation scheme [17]. Thus, formal manipulations like the insertion of the action (3.2) become well justified.

The simplest case $k_{i}=2$, i.e., the four-point correlator of stress-tensor multiplets, has been treated in detail in Refs. [15, 18, 19] in the $\mathcal{N}=2$ framework (see also Appendix $\mathrm{A}$ for a short summary). The important result is that this correlator is determined by a single function of the conformal cross-ratios, and not by two functions, as predicted by the general analysis in Section 6. Conversely, this $\mathcal{N}=2$ result can be translated back in terms of the $\mathcal{N}=4$ insertion procedure. In order to be consistent with the $\mathcal{N}=2$ analysis, the relevant term in the corresponding five-point nilpotent covariant in (3.2) must have the following general form (after setting $\theta_{1}=\ldots=\theta_{4}=0$ ):

$$
\left\langle\mathcal{O}^{(2)}(0) \mathcal{O}^{(2)} \mathcal{O}^{(2)} \mathcal{O}^{(2)} \mathcal{O}^{(2)}\right\rangle=R^{2222}\left(\theta_{0}\right)^{4} F\left(x_{0}, \ldots, x_{4}\right)
$$

with

$$
\begin{aligned}
R^{2222}= & \frac{(12)^{2}(34)^{2}}{x_{12}^{4} x_{34}^{4}} s+\frac{(13)^{2}(24)^{2}}{x_{13}^{4} x_{24}^{4}}+\frac{(14)^{2}(23)^{2}}{x_{14}^{4} x_{23}^{4}} t+\frac{(13)(14)(23)(24)}{x_{13}^{2} x_{14}^{2} x_{23}^{2} x_{24}^{2}}(s-t-1) \\
& +\frac{(12)(14)(23)(34)}{x_{12}^{2} x_{14}^{2} x_{23}^{2} x_{34}^{2}}(1-s-t)+\frac{(12)(13)(24)(34)}{x_{12}^{2} x_{13}^{2} x_{24}^{2} x_{34}^{2}}(t-s-1) \\
= & \frac{1}{x_{13}^{2} x_{24}^{2}}\left[\frac{(12)^{2}(34)^{2}}{x_{12}^{2} x_{34}^{2}}+\frac{(13)(14)(23)(24)}{x_{13}^{2} x_{14}^{2} x_{23}^{2} x_{24}^{2}}\left(x_{12}^{2} x_{34}^{2}-x_{14}^{2} x_{23}^{2}-x_{13}^{2} x_{24}^{2}\right)+\text { cycle }\right]
\end{aligned}
$$

Note that the second form of the prefactor $R^{2222}$ is manifestly cyclic (i.e., crossing) symmetric, apart from the factor $1 / x_{13}^{2} x_{24}^{2}$. This results in the following properties of $R^{2222}$ under point permutations:

$$
\begin{array}{ll}
1 \leftrightarrow 2: & R^{2222} \rightarrow \frac{1}{t} R^{2222}, \\
1 \leftrightarrow 3: & R^{2222} \rightarrow R^{2222} .
\end{array}
$$

It is important to realize that the prefactor $R^{2222}$ does not depend on the coordinates, in particular, on the harmonics at the insertion point, so it is an $\mathrm{SO}(6)$ singlet at this point. At the same time, $R^{2222}$ has absorbed the entire harmonic dependence (i.e., the $\mathrm{SO}(6)$ irreps) at points 1 to 4 , while the harmonic dependence at point 0 is contained in the chiral Grassmann factor $\left(\theta_{0}\right)^{4}$. Consequently, the only part of the amplitude (3.3) which is not fixed by superconformal invariance, the function $F\left(x_{0}, \ldots, x_{4}\right)$, is not allowed to depend on any of the harmonics (in an analytic, i.e., polynomial fashion). Thus, this coefficient function is an $\mathrm{SO}(6)$ singlet.

Substituting (3.3) in (3.2) and integrating over $\theta_{0}$ (the Grassmann superaction measure in (3.2) exactly matches the nilpotent factor $\left(\theta_{0}\right)^{4}$ in (3.3)) and over $x_{0}$ at the insertion point, we obtain the allowed form of the quantum part of the four-point correlator:

$$
\frac{\partial}{\partial g^{2}}\left\langle\mathcal{O}^{\left(k_{1}\right)} \mathcal{O}^{\left(k_{2}\right)} \mathcal{O}^{\left(k_{3}\right)} \mathcal{O}^{\left(k_{4}\right)}\right\rangle=R^{2222} \mathcal{F}(s, t)
$$


Here the four-point conformal invariant

$$
\mathcal{F}(s, t)=\int \mathrm{d}^{4} x_{0} F\left(x_{0}, \ldots, x_{4}\right)
$$

satisfies the crossing symmetry conditions

$$
\mathcal{F}(s, t)=\mathcal{F}(t, s)=1 / t \mathcal{F}(s / t, 1 / t)
$$

as it easily follows from (3.5).

The prediction of conformal supersymmetry (kinematics) combined with the insertion formula (dynamics) consists in fixing the relative coefficients of all six terms in (3.4), leaving undetermined the single conformal invariant $\mathcal{F}(s, t)$. This should be compared to the two arbitrary functions allowed by the general arguments in Section 6 for $k=2$, see (2.10), (2.11) and (2.12). The single function $\mathcal{F}(s, t)$ encodes the dynamical information about the quantum part of the correlator. This phenomenon was revealed in Ref. [19] and was called "partial non-renormalisation". The predicted form has been confirmed by a number of explicit calculations: perturbative at order $g^{2}$ [20]-21] and $g^{4}$ [22, 23], instanton [24] and AdS supergravity [25], [19].

The generalisation of the $\mathcal{N}=4 \rightarrow \mathcal{N}=2$ projection procedure to arbitrary $k_{i} \geq 2$ becomes cumbersome, due to the increasing number of terms in the tensor product $\left[0, k_{i}, 0\right] \times$ $\left[0, k_{j}, 0\right]$ and to the large amount of linear algebra needed to reconstruct the $\mathcal{N}=4$ amplitude from its $\mathcal{N}=2$ projections. ${ }^{4}$ Here we choose an indirect way which efficiently exploits the knowledge from the simplest case $k_{i}=2$. We assume that the five-point nilpotent covariant in (3.2) can always be factorised into a "kernel" with $k_{i}=2$ and an extra factor carrying the rest of the $\mathrm{SO}(6)$ quantum numbers $k_{i}^{\prime}=k_{i}-2$ at each point:

$$
\left\langle\mathcal{O}^{(2)}(0) \mathcal{O}^{\left(k_{1}\right)} \mathcal{O}^{\left(k_{2}\right)} \mathcal{O}^{\left(k_{3}\right)} \mathcal{O}^{\left(k_{4}\right)}\right\rangle=R^{2222}\left(\theta_{0}\right)^{4} F^{k_{1}^{\prime} k_{2}^{\prime} k_{3}^{\prime} k_{4}^{\prime}}\left(x_{0}, \ldots, x_{4} ; u_{1}, \ldots, u_{4}\right) .
$$

The main difference from (3.3) is that now the factor $F^{k_{1}^{\prime} k_{2}^{\prime} k_{3}^{\prime} k_{4}^{\prime}}$ depends on the harmonic variables $u_{1}, \ldots, u_{4}$, i.e., it is not an $\mathrm{SO}(6)$ singlet at points 1 to 4 . The Grassmann factor $\left(\theta_{0}\right)^{4}$ still carries the full harmonic dependence at the insertion point. Thus, the functional freedom in (3.9) and, after integration over the insertion point, in the quantum correction (3.2) is determined by the $\mathrm{SO}(6)$ structure of the factor $F^{k_{1}^{\prime}} k_{2}^{\prime} k_{3}^{\prime} k_{4}^{\prime}$.

Take for example arbitrary $k_{1}, k_{2}$ and $k_{3}$, but keep $k_{4}=2$. The factor $F^{k_{1}^{\prime} k_{2}^{\prime} k_{3}^{\prime} 0}$ still contains a unique harmonic structure:

$$
F^{k_{1}^{\prime} k_{2}^{\prime} k_{3}^{\prime} 0}=\left(\frac{(12)}{x_{12}^{2}}\right)^{\frac{1}{2}\left(k_{1}+k_{2}-k_{3}-2\right)}\left(\frac{(13)}{x_{13}^{2}}\right)^{\frac{1}{2}\left(k_{1}+k_{3}-k_{2}-2\right)}\left(\frac{(23)}{x_{23}^{2}}\right)^{\frac{1}{2}\left(k_{2}+k_{3}-k_{1}-2\right)} f\left(x_{0}, \ldots, x_{4}\right),
$$

where $f$ is an arbitrary $\mathrm{SO}(6)$ singlet function. After integration over $\theta_{0}, x_{0}$ we again find that the amplitude is determined by one function, $\phi(s, t)=\int \mathrm{d}^{4} x_{0} f\left(x_{0}, \ldots, x_{4}\right)$, just like in (3.7).

Next we move to the main case of interest in this paper, $k_{i}=3 \rightarrow k_{i}^{\prime}=1$. According to Section [6] the factor $F^{1111}$ contains three terms:

$$
F^{1111}=\frac{(12)(34)}{x_{12}^{2} x_{34}^{2}} \alpha\left(x_{0}, \ldots, x_{4}\right)+\frac{(14)(23)}{x_{14}^{2} x_{23}^{2}} \beta\left(x_{0}, \ldots, x_{4}\right)+\frac{(13)(24)}{x_{13}^{2} x_{24}^{2}} \gamma\left(x_{0}, \ldots, x_{4}\right) .
$$

\footnotetext{
${ }^{4}$ For sufficiently low values of $k$ this is still doable, as we show in Appendix A for $k=3$.
} 
After integration over the insertion point $x_{0}$ the coefficients in (3.11) give rise to four-point conformal invariants, $\alpha(s, t), \beta(s, t), \gamma(s, t)$. Inserting all this back into (3.9) and then into (3.2) and using the explicit form (3.4) of $R^{2222}$, we obtain the following factorised form of the quantum part of the four-point correlator:

$$
\begin{aligned}
\frac{\partial}{\partial g^{2}}\left\langle\mathcal{O}^{(3)} \mathcal{O}^{(3)} \mathcal{O}^{(3)} \mathcal{O}^{(3)}\right\rangle=R^{2222} F^{1111} \\
=\left[\frac{(12)^{2}(34)^{2}}{x_{12}^{4} x_{34}^{4}} s+\frac{(13)^{2}(24)^{2}}{x_{13}^{4} x_{24}^{4}}+\frac{(14)^{2}(23)^{2}}{x_{14}^{4} x_{23}^{4}} t+\frac{(13)(14)(23)(24)}{x_{13}^{2} x_{14}^{2} x_{23}^{2} x_{24}^{2}}(s-t-1)\right. \\
\left.\quad+\frac{(12)(14)(23)(34)}{x_{12}^{2} x_{14}^{2} x_{23}^{2} x_{34}^{2}}(1-s-t)+\frac{(12)(13)(24)(34)}{x_{12}^{2} x_{13}^{2} x_{24}^{2} x_{34}^{2}}(t-s-1)\right] \\
\quad \times\left[\frac{(12)(34)}{x_{12}^{2} x_{34}^{2}} \alpha+\frac{(14)(23)}{x_{14}^{2} x_{23}^{2}} \beta+\frac{(13)(24)}{x_{13}^{2} x_{24}^{2}} \gamma\right] .
\end{aligned}
$$

Doing the multiplication and bringing the result to the standard form (2.14), we may express the ten coefficients $a, b, c$ in terms of only three functions:

$$
\begin{aligned}
& a_{1}=s \alpha(s, t), \quad a_{2}=\gamma(s, t), \quad a_{3}=t \beta(s, t) ; \\
& b_{1}=s \gamma+(t-s-1) \alpha \\
& b_{2}=s \beta+(1-s-t) \alpha \\
& b_{3}=(s-t-1) \beta+t \gamma \\
& b_{4}=t \alpha+(1-s-t) \beta \\
& b_{5}=\alpha+(t-s-1) \gamma \\
& b_{6}=\beta+(s-t-1) \gamma \\
& c=(s-t-1) \alpha+(t-s-1) \beta+(1-s-t) \gamma .
\end{aligned}
$$

We still have to impose the crossing symmetry condition on the correlator (3.9) with respect to points $1, \ldots, 4$. Taking into account the property (3.5) of the prefactor $R^{2222}$, it is easy to derive that the coefficients $\alpha(s, t), \beta(s, t), \gamma(s, t)$ are not independent:

$$
\alpha(s, t)=1 / s \gamma(t / s, 1 / s), \quad \beta(s, t)=1 / t \gamma(s / t, 1 / t), \quad \gamma(s, t)=\gamma(t, s) .
$$

Thus, all the ten coefficients in the quantum part of the amplitude (2.14) are expressed in terms of a single function, for instance, $\alpha(s, t)$ obeying the symmetry relation $\alpha(s / t, 1 / t)=$ $t \alpha(s, t)$. This is the content of the partial non-renormalisation theorem for the correlator of four $\frac{1}{2}$-BPS operators of weight 3 .

Clearly, the same analysis can be carried out for correlators with arbitrary weights $k_{i}$. The effect of the insertion procedure is to reduce the weight at each point by two units, $k_{i} \rightarrow k_{i}^{\prime}=k_{i}-2$. The resulting four-point object $F^{k_{1}^{\prime} k_{2}^{\prime} k_{3}^{\prime} k_{4}^{\prime}}$ depends on as many functions as predicted by its $\mathrm{SO}(6)$ structure (and eventually by crossing symmetry). For example, if $k_{i}=4$ initially the correlator can depend on four functions (see the end of Section [6). However, in this case $k_{i}^{\prime}=2$ and we know from Section [6 that the fully crossing symmetric factor $F^{2222}$ only involves two functions. The conclusion is that the quantum part of the weight four correlator can only involve two independent functions. 
Concluding this section, we mention that a somewhat different approach presented in the recent paper [8] leads to the same number of independent functions in an amplitude of weights $k_{1}=\ldots=k_{4}=k$. Instead of applying directly the insertion procedure to the fourpoint correlators, the authors rely on their earlier result [7] on the non-renormalisation of three-point functions of all kinds of protected (BPS or semishort) operators. Combining this with double-OPE arguments of the type developed in Ref. [26], they are able to predict the same general form of the amplitude. It should however be stressed that the nonrenormalisation theorem of Ref. [7] is also based on the insertion procedure, but this time at the level of three-point rather than four-point functions. Another important remark is that this non-renormalisation theorem cannot be directly tested in supergravity simply because the supergravity spectrum has only fields dual to $\frac{1}{2}$-BPS short operators.

\section{Supergravity-induced four-point amplitude}

In this section we provide a novel example of a conformal four-point amplitude induced by type IIB supergravity on an $A d S_{5} \times S^{5}$ background. So far the knowledge of the stronglycoupled four-point functions [27, 25] has been limited to those of the stress-tensor multiplet dual to the field content of gauged $\mathcal{N}=8$ supergravity. We thus provide for the first time a four-point amplitude for operators corresponding to higher KK states and verify its compatibility with the field-theoretic predictions.

The computation of the supergravity-induced correlation functions proceeds in the standard way. One first evaluates the on-shell gravity action $S$ with Dirichlet boundary conditions on the fields; then, varying the generating functional $Z=e^{-S}$ with respect to the boundary data one obtains correlation functions of the boundary CFT. In particular, the derivation of the four-point amplitude requires the knowledge of the effective supergravity action on $A d S_{5}$ up to fourth order in the fields. The quadratic and cubic supergravity terms needed to compute the four-point function of arbitrary $\frac{1}{2}$-BPS operators were found in [28-31]. Their contribution to the extrema of the supergravity action is interpreted as the AdS exchange graphs. The most difficult part of this program is however to obtain the quartic effective action corresponding to contact interactions. This formidable problem was solved in 32, where it was found in particular that the quartic Lagrangian contains derivative interactions (up to four derivatives).

The $\frac{1}{2}$-BPS operator $\mathcal{O}^{I}$ in the irrep $[0,3,0]$ (or 50) is dual to the gravity scalar $s^{I}$ with AdS mass $m^{2}=-3$. The relevant part of the effective five-dimensional action from [32] is given by

$$
S=\frac{N^{2}}{8 \pi^{2}} \int \mathrm{d}^{5} z \sqrt{g_{a}}\left(\mathcal{L}_{2}+\mathcal{L}_{3}+\mathcal{L}_{4}\right)
$$

i.e. it is a sum of quadratic, cubic and quartic terms. Here $g_{a}$ is the determinant of the Euclidean AdS metric $\mathrm{d} s^{2}=\frac{1}{z_{0}^{2}}\left(\mathrm{~d} z_{0}^{2}+\mathrm{d} x_{a} \mathrm{~d} x_{a}\right), a=1,2,3,4$.

To distinguish supergravity scalars and vectors belonging to different $\mathrm{SO}(6)$ representations we introduce the subscript $k$, i.e. $s_{k}^{I}$ (irrep $\left.[0, k, 0]\right), A_{\mu, k}^{I}$ (irrep $[1, k-1,1], k$ odd). It is related to the conformal dimension of the corresponding CFT operator as $\Delta=k$ for scalars and $\Delta=k+2$ for vectors. Then the Lagrangian involves the scalar fields $s_{2}^{I}, s_{3}^{I}$ and $s_{4}^{I}$, as well as two vectors $A_{\mu, 1}^{I}, A_{\mu, 3}^{I}$, the graviton $\phi_{\mu \nu}$ and a massive symmetric tensor $\varphi_{\mu \nu}^{I}$ 
transforming in the irrep $[0,2,0]$ of $\mathrm{SO}(6)$. Its quadratic part is normalised as follows ${ }^{5}$

$$
\begin{aligned}
\mathcal{L}_{2}= & \frac{1}{4}\left(\nabla_{\mu} s_{2}^{1} \nabla^{\mu} s_{2}^{1}-4 s_{2}^{1} s_{2}^{1}\right)+\frac{1}{4}\left(\nabla_{\mu} s_{3}^{1} \nabla^{\mu} s_{3}^{1}-3 s_{3}^{1} s_{3}^{1}\right)+\frac{3}{2} \nabla_{\mu} s_{4}^{1} \nabla^{\mu} s_{4}^{1} \\
& +\frac{1}{2}\left(F_{\mu \nu, 1}^{1}\right)^{2}+\frac{1}{2}\left(F_{\mu \nu, 3}^{1}\right)^{2}+8\left(A_{\mu, 3}^{1}\right)^{2} \\
& +\frac{1}{4} \nabla_{\rho} \phi_{\mu \nu} \nabla^{\rho} \phi^{\mu \nu}-\frac{1}{2} \nabla_{\mu} \phi_{\mu \rho} \nabla^{\nu} \phi_{\nu \rho}+\frac{1}{2} \nabla_{\mu} \phi_{\rho}^{\rho} \nabla_{\nu} \phi^{\mu \nu}-\frac{1}{4} \nabla_{\rho} \phi_{\mu}^{\mu} \nabla^{\rho} \phi_{\nu}^{\nu} \\
& -\frac{1}{2} \phi_{\mu \nu} \phi^{\mu \nu}+\frac{1}{2}\left(\phi_{\nu}^{\nu}\right)^{2} \\
& +6\left[\frac{1}{4} \nabla_{\rho} \varphi_{\mu \nu}^{1} \nabla^{\rho} \varphi^{\mu \nu 1}-\frac{1}{2} \nabla_{\mu} \varphi_{\mu \rho}^{1} \nabla^{\nu} \varphi_{\nu \rho}^{1}+\frac{1}{2} \nabla_{\mu} \varphi_{\rho}^{\rho 1} \nabla_{\nu} \varphi^{\mu \nu 1}-\frac{1}{4} \nabla_{\rho} \varphi_{\mu}^{\mu 1} \nabla^{\rho} \varphi_{\nu}^{\nu 1}\right. \\
& \left.+\frac{5}{2} \varphi_{\mu \nu}^{1} \varphi^{\mu \nu 1}-\frac{7}{2}\left(\varphi_{\nu}^{\nu}\right)^{2}\right],
\end{aligned}
$$

(summation over the repeated superscript indices is implied).

To write down the cubic Lagrangian we introduce the so-called C-tensors. They are the Clebsh-Gordon coefficients for tensor products of different $\mathrm{SO}(6)$ irreps and describe the cubic interactions of various supergravity fields. For all necessary definitions and the properties of the C-tensors we refer the reader to Appendix $\mathrm{B}$ where in particular the summation formulae which we term as "C-algebra" are established. Explicitly, the cubic Lagrangian is

$$
\begin{aligned}
\mathcal{L}_{3}= & -3\left\langle C^{1} C^{2} C_{[0,2,0]}^{3}\right\rangle s_{3}^{1} s_{3}^{2} s_{2}^{3}-18\left\langle C^{1} C^{2} C_{[0,4,0]}^{3}\right\rangle s_{3}^{1} s_{3}^{2} s_{4}^{3} \\
& -\frac{1}{4}\left(\nabla^{\mu} s_{3}^{1} \nabla^{\nu} s_{3}^{1} \phi_{\mu \nu}-\frac{1}{2}\left(\nabla^{\mu} s_{3}^{1} \nabla_{\mu} s_{3}^{1}-3 s_{3}^{1} s_{3}^{1}\right) \phi_{\nu}^{\nu}\right) \\
& -\frac{3}{4}\left\langle C^{1} C^{2} C_{[0,2,0]}^{3}\right\rangle\left(\nabla^{\mu} s_{3}^{1} \nabla^{\nu} s_{3}^{2} \varphi_{\mu \nu}^{3}-\frac{1}{2}\left(\nabla^{\mu} s_{3}^{1} \nabla_{\mu} s_{3}^{2}-9 s_{3}^{1} s_{3}^{2}\right) \varphi_{\nu}^{\nu}{ }^{3}\right) \\
& -\frac{3}{2}\left\langle C^{1} C^{2} C_{[1,0,1]}^{3}\right\rangle s_{3}^{1} \nabla^{\mu} s_{3}^{2} A_{\mu, 1}^{3}-3\left\langle C^{1} C^{2} C_{[1,2,1]}^{3}\right\rangle s_{3}^{1} \nabla^{\mu} s_{3}^{2} A_{\mu, 3}^{3} .
\end{aligned}
$$

From this Lagrangian we deduce that the interactions of the scalar field $s_{3}$ are mediated by two neighboring multiplets: one of them is the massless graviton multiplet $\left(s_{2}^{I}, A_{\mu, 1}^{I}, \phi_{\mu \nu}\right)$ which includes the $m^{2}=-4$ scalar, the massless vector and the graviton and the massive multiplet $\left(s_{4}^{I}, A_{\mu, 3}^{I}, \varphi_{\mu \nu}^{I}\right)$ whose lowest component is the massless scalar $s_{4}$.

Since the leading (i.e. most singular) and the subleading terms in the OPE are determined by the three-point functions derived from the cubic Lagrangian, the cubic couplings (4.3) are responsible for these contributions to the supergravity-induced OPE. Thus, in the leading and the subleading terms in the OPE we expect the following irreps to appear: leading $[0,0,0],[1,0,1]$ and $[0,2,0]$ corresponding to the stress tensor, the conserved current and a $\frac{1}{2}$-BPS operator with conformal dimension $\Delta=2$; subleading $[0,4,0],[1,2,1]$ and $[0,2,0]$ corresponding to a $\frac{1}{2}$-BPS operator with $\Delta=4$, and higher vector and tensor currents, respectively. In principle, one could also expect the appearance of a cubic interaction with a $\frac{1}{2}$-BPS multiplet whose lowest component is a scalar of $\Delta=6$. However, the corresponding coupling is then extremal and therefore it must vanish [33, 34. The representations which do not appear among the cubic couplings correspond, in free-theory terms, to the contribution of the double-trace operators.

\footnotetext{
${ }^{5}$ To simplify the action we have performed suitable rescalings of the fields.
} 

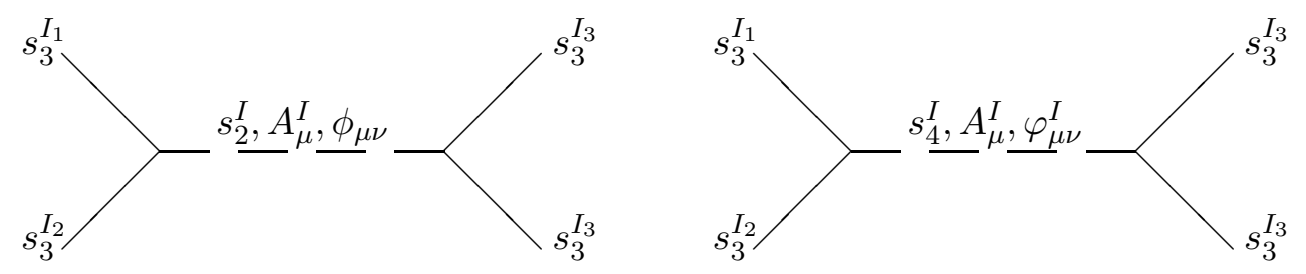

Figure 4: Exchange graphs contributing to the four-point function of operators $\mathcal{O}^{I}$. The first graph represents the exchange of fields from the massless graviton multiplet: the scalar $s_{2}$ in the 20, the vector $A_{\mu}$ in the $\mathbf{1 5}$ and the graviton $\phi_{\mu \nu}$. The other graph involves a massive multiplet whose lowest scalar component $s_{4}$ is in the $\mathbf{1 0 5}$. These graphs are responsible for the leading and the subleading terms in the OPE.

One of the most involved parts of our computation is to extract the relevant contact interactions from the general quartic action of Ref. 32. This action contains the quartic terms with two and four derivatives as well as terms without derivatives and for generic fields $s^{I}$ looks pretty complicated (see Appendix A of Ref. [32]). However, by heavy usage of the C-algebra, the symmetry properties of the derivative vertices and integration by parts (see Appendix [C for details) we reduced it to the remarkably simple expression

$$
\mathcal{L}_{4}=\left(\frac{3}{2} S^{1234}+\frac{39}{32} C^{1234}-\frac{3}{16} \delta^{12} \delta^{34}\right) s^{1} s^{2} s^{3} s^{4}-\frac{3}{32} C^{1234} s^{1} \nabla_{\mu} s^{2} \nabla^{\mu} s^{3} s^{4} .
$$

It is worthwhile noting that the quartic terms with four derivatives completely disappear, i.e. the final action is of the sigma model type. This suggests the interesting possibility that the quartic four-derivative Lagrangian of Ref. 32 is intrinsically zero, although this is not seen for generic fields, i.e. without specifying the explicit representation content. If true, this might imply that the extension of the the five-dimensional gauged $\mathcal{N}=8$ supergravity by including the massive KK modes of the type IIB supergravity compactification is described by some sigma model.

Having established the relevant supergravity Lagrangian we can now proceed to the evaluation of the AdS exchange graphs. In comparison to other calculations existing in the literature [27, 25, 35], a novelty is the appearance of a massive symmetric tensor. The corresponding exchange graph can be computed by generalizing the method of Ref. [36] (see Appendix E). Omitting the details of the tedious calculations, here we present the final result for the supergravity-induced four-point function of the canonically normalised $\frac{1}{2}$-BPS operators of dimension 3 :

$$
\begin{aligned}
& \left\langle\mathcal{O}^{1}\left(x_{1}\right) \ldots \mathcal{O}^{4}\left(x_{4}\right)\right\rangle=\frac{\delta^{12} \delta^{34}}{x_{12}^{6} x_{34}^{6}} \\
& \quad+\frac{72}{\pi^{2} N^{2}}\left[S^{1234} A_{1234}^{S}+\delta^{12} \delta^{34} A_{1234}^{\delta}+C^{1234} A_{1234}^{C}+C^{1243} A_{1243}^{C}\right]+t+u .
\end{aligned}
$$


Here we explicitly exhibit only the expression in the $s$-channel as the $t$-channel is obtained by replacing $1 \leftrightarrow 4$, and the $u$-channel by $1 \leftrightarrow 3$. The terms without $1 / N^{2}$ in front represent the contribution of the disconnected AdS graphs. The coefficients $A_{1234}$ are expressed in terms of the $D$-functions (see Appendix D) as follows

$$
\begin{aligned}
A_{1234}^{S}= & 8\left(\frac{D_{3322}}{x_{34}^{2}}-\frac{2}{3} D_{3333}\right) \\
A_{1234}^{\delta}= & \frac{3}{4}\left(x_{13}^{2} x_{24}^{2}+x_{14}^{2} x_{23}^{2}-x_{12}^{2} x_{34}^{2}\right)\left(\frac{D_{4433}}{x_{34}^{2}}+\frac{D_{4422}}{x_{34}^{4}}\right)-\frac{1}{2} \frac{D_{3311}}{x_{34}^{4}}+\frac{5}{3} D_{3333} \\
A_{1234}^{C}= & \frac{3}{4}\left(x_{13}^{2} x_{24}^{2}+x_{14}^{2} x_{23}^{2}-x_{12}^{2} x_{34}^{2}\right) \frac{D_{4433}}{x_{34}^{2}}+\frac{D_{3311}}{x_{34}^{4}}-\frac{D_{3322}}{x_{34}^{2}}+2 \frac{D_{3232}}{x_{24}^{2}}-3 x_{14}^{2} D_{4334} \\
& -\frac{2}{x_{24}^{2}}\left(D_{3243} x_{34}^{2}-D_{3342} x_{23}^{2}\right)-\frac{1}{2 x_{34}^{2}}\left(D_{3423} x_{24}^{2}-D_{3432} x_{23}^{2}\right) \\
& -\frac{3}{2 x_{34}^{4}}\left(D_{3412} x_{24}^{2}-D_{3421} x_{23}^{2}\right) .
\end{aligned}
$$

\section{$5 \quad$ Verifying the CFT predictions}

As argued in Section 3. the insertion formula, being a dynamical constraint on the theory, predicts that the general four-point amplitude (2.14) depends on one undetermined coefficient function $a_{2}(s, t)$, while the nine others are expressed in terms of it. Here we compare the supergravity-induced four-point amplitude obtained in the previous section to this field-theoretic prediction and indeed find an almost miraculous agreement.

Initially we express all $D$-functions entering (4.6) in terms of corresponding functions $\bar{D}$ of the invariants $s, t$ (for a detailed definition see Appendix (D) so that the functions $c(s, t)$, $a_{1}(s, t)$ (the $1 / N^{2}$-part) and $b_{1}(s, t)$ can be represented as follows

$$
\begin{aligned}
c(s, t)= & \frac{2^{4} \cdot 9}{N^{2}} s t\left[-\frac{3}{2} \bar{D}_{3333}+\bar{D}_{2332}+\bar{D}_{3232}+\bar{D}_{3322}\right] \\
a_{1}(s, t)= & \frac{9}{2 N^{2}} s^{3}\left[(1+t-s)\left(\bar{D}_{4433}+\bar{D}_{4422}\right)+5 \bar{D}_{3333}-\bar{D}_{3311}\right] \\
b_{1}(s, t)= & \frac{9}{2 N^{2}} s^{2}\left[(1-s+t) \bar{D}_{4433}-4 \bar{D}_{4334}+t \bar{D}_{3432}-\bar{D}_{3423}\right. \\
& \left.+4 t \bar{D}_{3342}-4 \bar{D}_{3243}+2 t \bar{D}_{3421}-2 \bar{D}_{3412}+8 \bar{D}_{3232}-4 \bar{D}_{3322}+2 \bar{D}_{3311}\right]
\end{aligned}
$$

Our strategy is then similar to that in Refs. [19, 35. For integer $\Delta_{i}$ and $\sum_{i} \Delta_{i}$ even we may express $\bar{D}_{\Delta_{1} \Delta_{2} \Delta_{3} \Delta_{4}}(s, t)$ in terms of $s$ - and $t$-derivatives of the standard four-dimensional one-loop box integral $\Phi(s, t)=\bar{D}_{1111}(s, t)$. This representation is convenient since the derivatives $\partial_{s} \Phi(s, t)$ and $\partial_{t} \Phi(s, t)$ are expressed in a simple manner in terms of the function $\Phi(s, t)$ itself (see Appendix (D). Using the notation $\overline{\mathbf{D}}_{\Delta_{1} \Delta_{2} \Delta_{3} \Delta_{4}}$ to denote the appropriate differential operator we have

$$
\bar{D}_{\Delta_{1} \Delta_{2} \Delta_{3} \Delta_{4}}(s, t)=\overline{\mathbf{D}}_{\Delta_{1} \Delta_{2} \Delta_{3} \Delta_{4}} \Phi(s, t) .
$$

Substituting the explicit expressions for the $\overline{\mathbf{D}}$-operators from Appendix $\mathbf{D}$ and successively using the identities (D.8), we express all the coefficient functions via $\Phi(s, t)$. Upon 
substitution of our findings into eqs.(3.14) and (3.15), and use of the symmetry properties

$$
\Phi(s, t)=\Phi(t, s), \quad \Phi(s / t, 1 / t)=t \Phi(s, t), \quad \Phi(t / s, 1 / s)=s \Phi(s, t)
$$

we finally get

$$
\begin{aligned}
& b_{1}-s \gamma-(t-s-1) \alpha=\frac{9}{N^{2}}, \\
& c-(s-t-1) \alpha-(t-s-1) \beta-(1-s-t) \gamma=\frac{18}{N^{2}} .
\end{aligned}
$$

The r.h.s. of these formulae literally coincide with the free field theory values of the coefficients $b_{1}$ and $c$ in eq.(2.17)! We thus observe the surprising property of the supergravityinduced four-point amplitude to split into a "free" and a "quantum" parts, precisely reproducing the form predicted by SCFT. Moreover, the numerical coefficients in the "free" part of this amplitude are exactly those given by the free Wick contractions. What makes this so unexpected is that contrary to the SYM theory, the supergravity action has no coupling constant and therefore no natural separation of the free part from the interacting part. Yet, we observe the same splitting in the four-point amplitudes derived from this action. Thus, supergravity retains memory of the Lagrangian formulation of the gauge theory even with an infinite value of the 't Hooft coupling. In the absence of an obvious explanation why the supergravity-induced amplitude has this particular form, we consider this fact as a strong evidence in favor of the AdS/CFT duality conjecture. At the same time, our result is a very non-trivial check of the effective action derived in [32].

Finally, we note that in fact the results for the coefficient functions given in (5.1) can be drastically simplified by using various identities for the $\bar{D}$-functions. In Appendix D we present an independent proof of eqs.(5.4) based on the $\bar{D}$-algebra. In the process a surprisingly simple expression for $a_{1}(s, t)$ emerges:

$$
a_{1}(s, t)=-\frac{9}{N^{2}}\left(s^{2} \bar{D}_{3335}(s, t)+s \bar{D}_{2235}(s, t)\right) .
$$

According to our analysis in Section 3 this function can be taken as the unique function describing the supergravity-induced four-point amplitude of dimension 3 BPS operators. In the next Section we will use this representation to shed some light on the properties of the corresponding OPE.

\section{Operator Product Expansion}

The operator product expansion of the stress-energy tensor multiplets in $\mathcal{N}=4$ theory and the $A d S_{5}$ supergravity is by now a well-developed subject [37]-41], 26]. Here we will exhibit some general properties of the OPE underlying the supergravity four-point amplitude for our new example of dimension 3 operators.

\subsection{OPE and anomalous dimensions of some long multiplets}

For the purposes of analyzing the operator product expansion we may expand the four-point function in the form

$$
\left\langle\mathcal{O}^{1}\left(x_{1}\right) \mathcal{O}^{2}\left(x_{2}\right) \mathcal{O}^{3}\left(x_{3}\right) \mathcal{O}^{4}\left(x_{4}\right)\right\rangle=\frac{1}{x_{12}^{6} x_{34}^{6}} \sum_{\mathcal{J}} A_{\mathcal{J}}(s, t) P_{\mathcal{J}}^{1234},
$$


and using the explicit formulae for the projectors $P_{\mathcal{J}}^{1234}$ from Appendix B we may write

$$
\begin{aligned}
A_{[0,0,0]}= & 50 a_{1}+s^{3} a_{2}+\frac{s^{3}}{t^{3}} a_{3}+\frac{25}{3}\left(s b_{1}+\frac{s}{t} b_{2}\right)+\frac{5}{2}\left(s^{2} b_{5}+\frac{s^{2}}{t^{2}} b_{4}\right) \\
& +\frac{1}{6}\left(\frac{s^{3}}{t} b_{6}+\frac{s^{3}}{t^{2}} b_{3}\right)+\frac{5}{6} \frac{s^{2}}{t} c, \\
A_{[1,0,1]}= & s^{3} a_{2}-\frac{s^{3}}{t^{3}} a_{3}+\frac{35}{9}\left(s b_{1}-\frac{s}{t} b_{2}\right)+\frac{35}{18}\left(s^{2} b_{5}-\frac{s^{2}}{t^{2}} b_{4}\right)+\frac{1}{18}\left(\frac{s^{3}}{t} b_{6}-\frac{s^{3}}{t^{2}} b_{3}\right), \\
A_{[0,2,0]}= & s^{3} a_{2}+\frac{s^{3}}{t^{3}} a_{3}+\frac{35}{12}\left(s b_{1}+\frac{s}{t} b_{2}\right)+\frac{7}{4}\left(s^{2} b_{5}+\frac{s^{2}}{t^{2}} b_{4}\right)+\frac{1}{12}\left(\frac{s^{3}}{t} b_{6}+\frac{s^{3}}{t^{2}} b_{3}\right)+\frac{7}{24} \frac{s^{2}}{t} c, \\
A_{[2,0,2]}= & s^{3} a_{2}+\frac{s^{3}}{t^{3}} a_{3}+\frac{10}{9}\left(s^{2} b_{5}+\frac{s^{2}}{t^{2}} b_{4}\right)-\frac{1}{9}\left(\frac{s^{3}}{t} b_{6}+\frac{s^{3}}{t^{2}} b_{3}\right)-\frac{5}{9} \frac{s^{2}}{t} c, \\
A_{[0,4,0]}= & s^{3} a_{2}+\frac{s^{3}}{t^{3}} a_{3}+\frac{7}{9}\left(s^{2} b_{5}+\frac{s^{2}}{t^{2}} b_{4}\right)+\frac{2}{9}\left(\frac{s^{3}}{t} b_{6}+\frac{s^{3}}{t^{2}} b_{3}\right)+\frac{7}{9} \frac{s^{2}}{t} c, \\
A_{[1,2,1]}= & s^{3} a_{2}-\frac{s^{3}}{t^{3}} a_{3}+s^{2} b_{5}-\frac{s^{2}}{t^{2}} b_{4}, \\
A_{[3,0,3]}= & s^{3} a_{2}-\frac{s^{3}}{t^{3}} a_{3}-\frac{1}{3}\left(\frac{s^{3}}{t} b_{6}-\frac{s^{3}}{t^{2}} b_{3}\right), \\
A_{[0,6,0]}= & s^{3} a_{2}+\frac{s^{3}}{t^{3}} a_{3}+\frac{s^{3}}{t} b_{6}+\frac{s^{3}}{t^{2}} b_{3}, \\
A_{[2,2,2]}= & s^{3} a_{2}+\frac{s^{3}}{t^{3}} a_{3}-\frac{1}{9}\left(\frac{s^{3}}{t} b_{6}+\frac{s^{3}}{t^{2}} b_{3}\right), \\
A_{[1,4,1]}= & s^{3} a_{2}-\frac{s^{3}}{t^{3}} a_{3}+\frac{1}{3}\left(\frac{s^{3}}{t} b_{6}-\frac{s^{3}}{t^{2}} b_{3}\right) .
\end{aligned}
$$

Using the expressions for $a_{i}, b_{i}$ and $c$ in terms of $\alpha, \beta, \gamma$ this may be simplified. For the purposes here it is convenient to define

$$
\mathcal{A}=\frac{s}{t} \alpha, \quad \mathcal{B}=\frac{s^{2}}{t^{2}}(t \gamma-\beta), \quad \mathcal{C}=\frac{s^{2}}{t^{2}}(t \gamma+\beta),
$$

and also to separate the results into two parts

$$
A_{\mathcal{J}}=A_{\mathcal{J}}^{\text {free }}+A_{\mathcal{J}}^{\text {int. }}
$$

where the first term corresponds to the result in free field theory

$$
\begin{aligned}
& A_{[0,6,0]}^{\text {free }}=s^{3}+\frac{s^{3}}{t^{3}}+\frac{9}{N^{2}}\left(\frac{s^{3}}{t}+\frac{s^{3}}{t^{2}}\right), \\
& A_{[1,4,1]}^{\text {free }}=s^{3}-\frac{s^{3}}{t^{3}}+\frac{3}{N^{2}}\left(\frac{s^{3}}{t}-\frac{s^{3}}{t^{2}}\right), \\
& A_{[2,2,2]}^{\text {free }}=s^{3}+\frac{s^{3}}{t^{3}}-\frac{1}{N^{2}}\left(\frac{s^{3}}{t}+\frac{s^{3}}{t^{2}}\right), \\
& A_{[3,0,3]}^{\text {free }}=s^{3}-\frac{s^{3}}{t^{3}}-\frac{3}{N^{2}}\left(\frac{s^{3}}{t}-\frac{s^{3}}{t^{2}}\right), \\
& A_{[0,4,0]}^{\text {free }}=s^{3}+\frac{s^{3}}{t^{3}}+\frac{2}{N^{2}}\left(\frac{s^{3}}{t}+\frac{s^{3}}{t^{2}}\right)+\frac{7}{N^{2}} s^{2}\left(1+\frac{1}{t}\right)^{2}, \\
& A_{[1,2,1]}^{\text {free }}=s^{3}-\frac{s^{3}}{t^{3}}+\frac{9}{N^{2}}\left(\frac{s^{2}}{t}-\frac{s^{2}}{t^{2}}\right),
\end{aligned}
$$




$$
\begin{aligned}
& A_{[2,0,2]}^{\text {free }}=s^{3}+\frac{s^{3}}{t^{3}}-\frac{1}{N^{2}}\left(\frac{s^{3}}{t}+\frac{s^{3}}{t^{2}}\right)+\frac{10}{N^{2}} s^{2}\left(1-\frac{1}{t}+\frac{1}{t^{2}}\right) \\
& A_{[0,2,0]}^{\text {free }}=s^{3}+\frac{s^{3}}{t^{3}}+\frac{3}{4 N^{2}}\left(\frac{s^{3}}{t}+\frac{s^{3}}{t^{2}}\right)+\frac{63}{4 N^{2}} s^{2}\left(1+\frac{1}{3 t}+\frac{1}{t^{2}}\right)+\frac{105}{4 N^{2}} s\left(1+\frac{1}{t}\right), \\
& A_{[1,0,1]}^{\text {free }}=s^{3}-\frac{s^{3}}{t^{3}}+\frac{1}{2 N^{2}} s\left(1-\frac{1}{t}\right)\left(70+35 s\left(1+\frac{1}{t}\right)+\frac{s^{2}}{t}\right), \\
& A_{[0,0,0]}^{\text {free }}=50+s^{3}+\frac{s^{3}}{t^{3}}+\frac{3}{2 N^{2}}\left(\frac{s^{3}}{t}+\frac{s^{3}}{t^{2}}\right)+\frac{45}{2 N^{2}} s^{2}\left(1+\frac{2}{3 t}+\frac{1}{t^{2}}\right)+\frac{75}{N^{2}} s\left(1+\frac{1}{t}\right)
\end{aligned}
$$

The remaining parts contain the essential dynamics

$$
\begin{aligned}
A_{[0,6,0]}^{\text {int. }}= & s^{2} \mathcal{C} \\
A_{[1,4,1]}^{\text {int. }}= & -\frac{2}{3}(1-t) s \mathcal{C}+\frac{1}{3} s^{2} \mathcal{B} \\
A_{[2,2,2]}^{\text {int. }}= & \frac{1}{9}(5(1+t)-s) s \mathcal{C}-\frac{5}{9}(1-t) s \mathcal{B}, \\
A_{[3,0,3]}^{\text {int. }}= & -\frac{1}{3}(1-t) s \mathcal{C}+\frac{1}{3}(3(1+t)-s) s \mathcal{B}, \\
A_{[0,4,0]}^{\text {int. }}= & \frac{1}{18}\left(14(1-t)^{2}-7 s(1+t)+4 s^{2}\right) \mathcal{C}-\frac{7}{18}(1-t) s \mathcal{B}+\frac{7}{9} s^{2} \mathcal{A}, \\
A_{[1,2,1]}^{\text {int. }}= & -\frac{1}{2}\left(1-t^{2}\right) \mathcal{C}+\frac{1}{2}(1-t)^{2} \mathcal{B}-(1-t) s \mathcal{A}, \\
A_{[2,0,2]}^{\text {int. }}= & \frac{1}{18}\left(5(1-t)^{2}+5 s(1+t)-2 s^{2}\right) \mathcal{C}-\frac{5}{18}(3(1+t)-s)(1-t) \mathcal{B} \\
& +\frac{5}{9}(3(1+t)-s) s \mathcal{A}, \\
A_{[0,2,0]}^{\text {int. }}= & \frac{1}{48}\left(35(1+t)^{2}-14(1-t)^{2}-27 s(1+t)+4 s^{2}\right) \mathcal{C} \\
& -\frac{1}{48}(35(1+t)-13 s)(1-t) \mathcal{B}+\frac{7}{24}\left(10(1-t)^{2}-5 s(1+t)+s^{2}\right) \mathcal{A}, \\
A_{[1,0,1]}^{\text {int. }}= & -\frac{1}{36}(35(1+t)-16 s)(1-t) \mathcal{C}+\frac{1}{36}\left(35(1+t)^{2}-20 s(1+t)+2 s^{2}\right) \mathcal{B} \\
& -\frac{35}{18}(2(1+t)-s)(1-t) \mathcal{A}, \\
A_{[0,0,0]}^{\text {int. }}= & \frac{1}{12}\left(25(1+t)^{2}-5(1-t)^{2}-15 s(1+t)+2 s^{2}\right) \mathcal{C}-\frac{5}{12}(2(1+t)-s)(1-t) \mathcal{B} \\
& +\frac{5}{6}\left(15(1+t)^{2}-5(1-t)^{2}-8 s(1+t)+s^{2}\right) \mathcal{A} .
\end{aligned}
$$

For the operator product expansion we expand $\mathcal{A}, \mathcal{B}$ and $\mathcal{C}$ in the form

$$
\sum_{\Delta, \ell} a_{\Delta, \ell} s^{\frac{1}{2}(\Delta-\ell)} G_{\Delta+4}^{(\ell)}(s, t)
$$

which represents them each as a sum of contributions of operators of scale dimension $\Delta$ and spin $\ell$, belonging to a $\left(\frac{1}{2} \ell, \frac{1}{2} \ell\right)$ spin representation. Explicit results for $G_{\Delta}^{(\ell)}(s, t)$ are known but here we note the important recurrence relations from [26],

$$
\begin{aligned}
-\frac{1}{2}(1-t) G_{\Delta}^{(\ell)}(s, t)= & G_{\Delta-1}^{(\ell+1)}(s, t)+\frac{1}{4} s G_{\Delta-1}^{(\ell-1)}(s, t)+\frac{1}{4} f(\Delta+\ell) s G_{\Delta+1}^{(\ell+1)}(s, t) \\
& +\frac{1}{16} f(\Delta-\ell-2) s^{2} G_{\Delta+1}^{(\ell-1)}(s, t) \\
\frac{1}{2}(1+t) G_{\Delta}^{(\ell)}(s, t)= & G_{\Delta-2}^{(\ell)}(s, t)+f(\Delta+\ell) s G_{\Delta}^{(\ell+2)}(s, t)+\frac{1}{4} s G_{\Delta}^{(\ell)}(s, t) \\
& +\frac{1}{16} f(\Delta-\ell-2) s^{2} G_{\Delta}^{(\ell-2)}(s, t) \\
& +\frac{1}{4} f(\Delta+\ell) f(\Delta-\ell-2) s^{2} G_{\Delta+2}^{(\ell)}(s, t)
\end{aligned}
$$


for $f(\lambda)=\frac{1}{4} \lambda^{2} /\left(\lambda^{2}-1\right)$. If we consider the contribution of a single term in the expansion of $\mathcal{A}, \mathcal{B}$ then using this in the above generates associated contributions in $A_{\mathcal{J}}$ for all operators expected for a long multiplet whose lowest dimension operator have scale dimension $\Delta$ and spin $\ell$ and which belong to the $[0,0,0],[1,0,1]$ representations respectively. In each case the expected representations with the appropriate $\Delta$ and spin $\ell$ arise, for the $[0,0,0]$ and $[1,0,1]$ cases the scale dimension varies from $\Delta$ to $\Delta+8$ and for scale dimension $\Delta+4$ the spin varies from $\ell-4$ to $\ell+4$ as expected. For $\mathcal{C}$ we would find that the set of operators contributing to the $[0,0,0]$ representation is not in accord with that expected for an operator based on the $[0,2,0]$ representation. This problem is easily cured by setting

$$
\mathcal{A}=\mathcal{A}^{\prime}-\frac{1}{6} \mathcal{C}
$$

which removes the $(1+t)^{2}$ term in the coefficient of $\mathcal{C}$ in $A_{[0,0,0]}$ in (6.6) which is the cause of this problem. This change then leads to

$$
\begin{aligned}
A_{[0,6,0]}^{\text {int. }}= & s^{2} \mathcal{C} \\
A_{[1,4,1]}^{\text {int. }}= & -\frac{2}{3}(1-t) s \mathcal{C}+\frac{1}{3} s^{2} \mathcal{B}, \\
A_{[2,2,2]}^{\text {int. }}= & \frac{1}{9}(5(1+t)-s) s \mathcal{C}-\frac{5}{9}(1-t) s \mathcal{B}, \\
A_{[3,0,3]}^{\text {int. }}= & -\frac{1}{3}(1-t) s \mathcal{C}+\frac{1}{3}(3(1+t)-s) s \mathcal{B}, \\
A_{[0,4,0]}^{\text {int. }}= & \frac{1}{54}\left(42(1-t)^{2}-21 s(1+t)+5 s^{2}\right) \mathcal{C}-\frac{7}{18}(1-t) s \mathcal{B}+\frac{7}{9} s^{2} \mathcal{A}^{\prime}, \\
A_{[1,2,1]}^{\text {int. }}= & -\frac{1}{6}(3(1+t)-s)(1-t) \mathcal{C}+\frac{1}{2}(1-t)^{2} \mathcal{B}-(1-t) s \mathcal{A}^{\prime}, \\
A_{[2,0,2]}^{\text {int. }}= & \frac{1}{54}\left(15(1-t)^{2}-s^{2}\right) \mathcal{C}-\frac{5}{18}(3(1+t)-s)(1-t) \mathcal{B} \\
& +\frac{5}{9}(3(1+t)-s) s \mathcal{A}^{\prime}, \\
A_{[0,2,0]}^{\text {int. }}= & \frac{1}{144}\left(105(1+t)^{2}-28(1-t)^{2}-46 s(1+t)+5 s^{2}\right) \mathcal{C} \\
& -\frac{1}{48}(35(1+t)-13 s)(1-t) \mathcal{B}+\frac{7}{24}\left(10(1-t)^{2}-5 s(1+t)+s^{2}\right) \mathcal{A}^{\prime}, \\
A_{[1,0,1]}^{\text {int. }}= & -\frac{1}{108}(35(1+t)-13 s)(1-t) \mathcal{C}+\frac{1}{36}\left(35(1+t)^{2}-20 s(1+t)+2 s^{2}\right) \mathcal{B} \\
& -\frac{35}{18}(2(1+t)-s)(1-t) \mathcal{A}^{\prime}, \\
A_{[0,0,0]}^{\text {int. }}= & \frac{1}{36}\left(10(1-t)^{2}-5 s(1+t)+s^{2}\right) \mathcal{C}-\frac{5}{12}(2(1+t)-s)(1-t) \mathcal{B} \\
& +\frac{5}{6}\left(15(1+t)^{2}-5(1-t)^{2}-8 s(1+t)+s^{2}\right) \mathcal{A}^{\prime} .
\end{aligned}
$$

The operators arising from $\mathcal{C}$ now correspond exactly to those present in a long multiplet with the lowest dimension operator belonging to a $[0,2,0]$ representation. It is convenient to write (6.10) succinctly as a linear function of $\mathcal{A}^{\prime}, \mathcal{B}, \mathcal{C}$,

$$
A_{\mathcal{J}}^{\text {int. }}=F_{\mathcal{J}}\left(\mathcal{A}^{\prime}, \mathcal{B}, \mathcal{C}\right) .
$$

The detailed form for $\mathcal{A}, \mathcal{B}, \mathcal{C}$ to leading order in $1 / N^{2}$ is

$$
\begin{aligned}
\mathcal{A}(s, t) & =-\frac{9}{N^{2}} s^{3}\left(\bar{D}_{3533}(s, t)+\bar{D}_{3522}(s, t)\right), \\
\mathcal{B}(s, t) & =\frac{9}{N^{2}} s^{3}\left(\bar{D}_{2532}(s, t)-\bar{D}_{2523}(s, t)\right), \\
\mathcal{C}(s, t) & =-\frac{9}{N^{2}}\left(s^{3}\left(2 \bar{D}_{3533}(s, t)-\bar{D}_{3351}(s, t)\right)+\frac{s^{2}}{t^{2}}\right)
\end{aligned}
$$


where we used the results in appendix D to express them in a form with the maximal overall power of $s$.

To analyse the operator product expansion for long multiplets we focus on $A_{\mathcal{J}}$ for $\mathcal{J}=[0,6,0],[1,4,1],[0,4,0]$. For these $\mathcal{J}$ it is convenient to define $\mathcal{A}_{0}, \mathcal{B}_{0}, \mathcal{C}_{0}$ such that $A_{\mathcal{J}}^{\text {free }}=F_{\mathcal{J}}\left(\mathcal{A}_{0}, \mathcal{B}_{0}, \mathcal{C}_{0}\right)$ to leading order in $1 / N^{2}$.

We first consider $A_{[0,6,0]}$. The free part determines $\mathcal{C}_{0}$ which has the expansion

$$
\mathcal{C}_{0}(s, t)=s+\frac{s}{t^{3}}=\sum_{\substack{\tau=1,2, \ldots \\ \ell=0,2, \ldots}} c_{\tau, \ell} s^{\tau} G_{2 \tau+\ell+4}^{(\ell)}(s, t)
$$

for

$$
c_{\tau, \ell}=2^{\ell-2} \frac{(\ell+\tau+1) !(\ell+\tau+2) ! \tau !(\tau+1) !}{(2 \ell+2 \tau+1) !(2 \tau) !} \tau(\ell+1)(\ell+2 \tau+2) .
$$

We may also obtain, by using the results of Appendix $\mathbb{E}$

$$
\begin{gathered}
\mathcal{C}(s, t) \sim-\frac{1}{N^{2}} \ln s \sum_{\substack{\tau=3,4, \ldots \\
\ell=0,2, \ldots}} \hat{c}_{\tau, \ell} s^{\tau} G_{2 \tau+\ell+4}^{(\ell)}(s, t), \\
\hat{c}_{\tau, \ell}=2^{\ell-2} \frac{(\ell+\tau) !(\ell+\tau+1) ! \tau !(\tau+3) !}{(2 \ell+2 \tau+1) !(2 \tau) !} \\
\times \tau(\tau-1)(\tau-2)((2 \ell+3 \tau+4)(\ell+\tau+1)-\ell \tau) .
\end{gathered}
$$

For $\tau=1,2$ there are no contributions involving $\ln s$ and the corresponding operators in the $[0,6,0]$ representation belong to protected semi-short multiplets. For $\tau=3,4, \ldots$, $\ell=0,2, \ldots$ the $\ln s$ contributions generate anomalous dimensions for long multiplets whose lowest dimension operator belongs to a $[0,2,0]$ with scale dimensions $\Delta_{\tau, \ell}=2 \tau+\ell+\eta_{\tau, \ell}$. Assuming there is just a single long multiplet for each $\tau, \ell$ the leading large $N$ contribution to $\eta_{\tau, \ell}$ is given by $-2 \hat{c}_{\tau, \ell} / c_{\tau, \ell} N^{2}$ giving

$$
\eta_{\tau, \ell}=-\frac{2}{N^{2}} \frac{(\tau+3)(\tau+2)(\tau-1)(\tau-2)}{(\ell+\tau+1)(\ell+\tau+2)(\ell+2 \tau+2)(\ell+1)}((2 \ell+3 \tau+4)(\ell+\tau+1)-\ell \tau) .
$$

The next step is to consider $A_{[1,4,1]}$ where the expansion of $\mathcal{B}$ gives results for anomalous dimensions for long multiplets with the lowest dimension operator belonging to a $[1,0,1]$ representation. From (6.5) and (6.10) the corresponding free contribution to leading order at large $N$, after removing the parts corresponding to the $[0,2,0]$ long supermultiplets, is given by defining $\mathcal{B}_{0}$ analogously to $\mathcal{C}_{0}$,

$$
\mathcal{B}_{0}(s, t)=3\left(s-\frac{s}{t^{3}}\right)+2(1-t)\left(1+\frac{1}{t^{3}}\right)=\sum_{\substack{\tau=0,1, \ldots \\ \ell=1,3, \ldots}} b_{\tau, \ell} s^{\tau} G_{2 \tau+\ell+4}^{(\ell)}(s, t),
$$

for

$$
b_{\tau, \ell}=2^{\ell-2} \frac{(\ell+\tau) !(\ell+\tau+1) !(\tau !)^{2}}{(2 \ell+2 \tau+1) !(2 \tau) !}(\tau-1)(\tau+2)(\ell+1)(\ell+2 \tau+2)(\ell+\tau)(\ell+\tau+3) .
$$


For the expansion of the $\ln s$ terms in $\mathcal{B}$ we use from (D.27)

$$
\begin{gathered}
\mathcal{B}(s, t) \sim \frac{9}{N^{2}} \ln s s^{3} \sum_{m, n=0}^{\infty} \frac{(m+2) !(m+n+3) !(m+n+5) !}{m ! n !(2 m+n+7) !} s^{m}(1-t)^{n+1} \\
=-\frac{1}{N^{2}} \ln s \sum_{\substack{\tau=3,4, \ldots \\
\ell=1,3, \ldots}} \hat{b}_{\tau, \ell} s^{\tau} G_{2 \tau+\ell+4}^{(\ell)}(s, t) \\
\hat{b}_{\tau, \ell}=2^{\ell-1} \frac{(\ell+\tau) !(\ell+\tau+1) ! \tau !(\tau+3) !}{(2 \ell+2 \tau+1) !(2 \tau) !} \tau(\tau-1)(\tau-2)(\ell+1)(\ell+2 \tau+2) .
\end{gathered}
$$

We may then read off the anomalous dimensions for $\tau=3,4 \ldots, \ell=1,3, \ldots$,

$$
\eta_{\tau, \ell}=-\frac{4}{N^{2}} \frac{(\tau+3)(\tau+1) \tau(\tau-2)}{(\ell+\tau)(\ell+\tau+3)} .
$$

From (6.18) $b_{0, \ell}<0$. However this case should not be taken in isolation as for the corresponding twist 4 operators it is necessary to analyse the contribution of semi-short multiplets which we undertake later.

The final step is a similar analysis of $A_{[0,4,0]}$ where the expansion of $\mathcal{A}^{\prime}$ yields anomalous dimensions for long multiplets with the lowest dimension operator belonging to the $[0,0,0]$ representation. After removing the free contributions of the $[0,2,0]$ and $[1,0,1]$ long multiplets then, from (6.5) and (6.10), the corresponding free contribution to leading order at large $N$ is given by

$$
\mathcal{A}_{0}(s, t)=\frac{1}{2}\left(1+t+\frac{7}{3} s\right)\left(1+\frac{1}{t^{3}}\right)+\frac{3}{2}(1-t)\left(1-\frac{1}{t^{3}}\right)=\sum_{\substack{\tau=0,1, \ldots \\ \ell=0,2, \ldots}} a_{\tau, \ell} s^{\tau} G_{2 \tau+\ell+4}^{(\ell)}(s, t),
$$

where

$$
\begin{aligned}
a_{\tau, \ell}= & 2^{\ell-3} \frac{(\ell+\tau) !(\ell+\tau+1) !(\tau !)^{2}}{3(2 \ell+2 \tau+1) !(2 \tau) !} \\
& \times(\tau-2)(\tau+3)(\ell+1)(\ell+2 \tau+2)(\ell+\tau-1)(\ell+\tau+4) .
\end{aligned}
$$

In a similar fashion as before we may find that for $\mathcal{A}^{\prime}$ we have

$$
\mathcal{A}^{\prime}(s, t) \sim-\frac{1}{N^{2}} \ln s \sum_{\substack{\tau=3,4, \ldots \\ \ell=0,2, \ldots}} \hat{a}_{\tau, \ell} s^{\tau} G_{2 \tau+\ell+4}^{(\ell)}(s, t),
$$

for

$$
\begin{aligned}
\hat{a}_{\tau, \ell}= & 2^{\ell-2} \frac{(\ell+\tau) !(\ell+\tau+1) ! \tau !(\tau+3) !}{3(2 \ell+2 \tau+1) !(2 \tau) !} \\
& \times \tau(\tau-1)(\tau-2)((\ell+\tau-1)(\ell+\tau+4)+5(\tau-2)(\tau+3)) .
\end{aligned}
$$

Taking these results into consideration then the anomalous dimensions for $\tau=3,4 \ldots$, $\ell=0,2, \ldots$, if there was a single long multiplet for each $\tau, \ell$, would be given by,

$$
\eta_{\tau, \ell}=-\frac{4}{N^{2}} \frac{(\tau+2)(\tau+1) \tau(\tau-1)}{(\ell+1)(\ell+2 \tau+2)}\left(1+\frac{5(\tau-2)(\tau+3)}{(\ell+\tau-1)(\ell+\tau+4)}\right) .
$$


From a similar analysis of the operator product expansion in 26] for the four point function of $[0,2,0] \frac{1}{2}$-BPS operators a result for $\eta_{\tau, \ell}$ was obtained without the final term in parentheses. For $\tau=2$ the result in [26] appears to be valid, and agrees with a similar calculation in [40, but for $\tau \geq 3$ the difference has to be a reflection of more than one long multiplet for each $\tau, \ell$ being present so that it is then necessary to consider operator mixing effects.

\subsection{Semi-short multiplets}

Besides the contributions of long multiplets which may have arbitrary scale dimensions greater than the unitarity bound there are also contributions from semi-short multiplets whose dimensions are protected. According to [5] the relevant semi-short multiplets which may contribute to the operator product expansion of two $[0,3,0] \frac{1}{2}$-BPS multiplets correspond to the case where the lowest dimension operator belongs to the representations $[0,0,0],[0,2,0],[2,0,2]$ and $[0,4,0]$, for even $\ell$, and $[1,0,1],[1,2,1]$ for odd $\ell$. Denoting the multiplets by $\mathcal{C}_{\mathcal{J} \ell}$ these contain operators with twist $\Delta-\ell$ according to the following table (obtained from [42]),

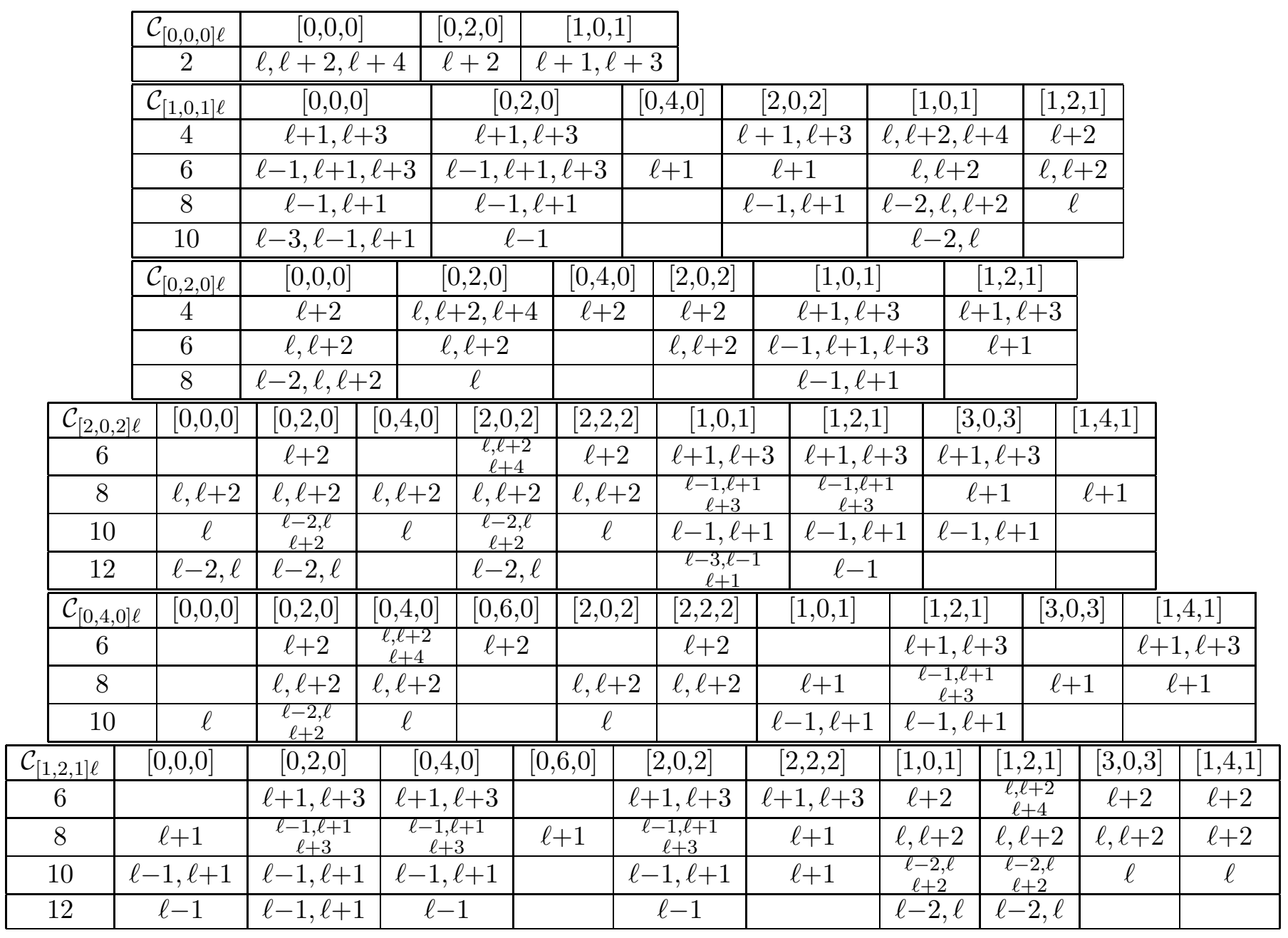


Table 1: Spins of operators for given twist belonging to semi-short multiplets contributing to the operator product expansion of two $[0,3,0] \frac{1}{2}$-BPS multiplets.

It is important to note that

$$
\mathcal{C}_{[0,0,0] \ell}+\mathcal{C}_{[1,0,1] \ell-1}, \quad \mathcal{C}_{[1,0,1] \ell}+\mathcal{C}_{[2,0,2] \ell-1}, \quad \mathcal{C}_{[0,2,0] \ell}+\mathcal{C}_{[1,2,1] \ell-1},
$$

combine to form complete long multiplets with the lowest scale dimension compatible with unitarity. These combinations may then be absorbed into contributions represented by $\mathcal{A}^{\prime}, \mathcal{B}, \mathcal{C}$ respectively, which may gain anomalous scale dimensions. Nevertheless the free field contributions given in (6.5) cannot all be described by a choice for $\mathcal{A}^{\prime}, \mathcal{B}, \mathcal{C}$ and so correspond to long multiplets. We show here, for simplicity to zeroth order in $1 / N$, how the additional terms necessary to accommodate (6.5) have restricted twists and are compatible with the expected contributions in Table 1 so that for suitable $\tau, \ell$,

$$
A_{\mathcal{J}}^{\text {short }}(s, t)=\sum_{\tau, \ell} d_{\tau, \ell}^{\mathcal{J}} s^{\tau+2} G_{2 \tau+\ell+4}^{(\ell)}(s, t) \text {. }
$$

To procede we first isolate those contributions to $\mathcal{C}_{0}, \mathcal{B}_{0}$ and $\mathcal{A}_{0}$ in (6.13), (6.17) and (6.21) which are protected in that there are no corresponding terms involving $\ln s$ which generate anomalous dimensions,

$$
\begin{aligned}
\mathcal{C}_{0}^{\text {short }}(s, t) & =\sum_{\tau=1,2} \sum_{\ell=0,2, \ldots} c_{\tau, \ell} s^{\tau} G_{2 \tau+\ell+4}^{(\ell)}(s, t), \\
\mathcal{B}_{0}^{\text {short }}(s, t) & =\sum_{\tau=0,2} \sum_{\ell=1,3, \ldots} b_{\tau, \ell} s^{\tau} G_{2 \tau+\ell+4}^{(\ell)}(s, t), \\
\mathcal{A}_{0}^{\text {short }}(s, t) & =\sum_{\tau=0,1} \sum_{\ell=0,2, \ldots} a_{\tau, \ell} s^{\tau} G_{2 \tau+\ell+4}^{(\ell)}(s, t),
\end{aligned}
$$

where we note that $b_{1, \ell}=c_{2, \ell}=0$. We then define, using (6.11),

$$
A_{\mathcal{J}}^{\text {short }}=F_{\mathcal{J}}\left(\mathcal{A}_{0}^{\text {short }}, \mathcal{B}_{0}^{\text {short }}, \mathcal{C}_{0}^{\text {short }}\right), \quad \mathcal{J}=[0,6,0],[1,4,1],[0,4,0] .
$$

Thus $A_{[0,6,0]}(s, t)=s^{2} \mathcal{C}_{0}^{\text {short }}(s, t)$ and it is easy to see that

$$
d_{\tau, \ell}^{[0,6,0]}=c_{\tau, \ell}, \quad \tau=1,2, \ell=0,2, \ldots .
$$

which corresponds to an operator product expansion involving protected twist 6 and 8 operators. The relevant operators may clearly be identified with the $\mathcal{C}_{[0,4,0] \ell}$ and $\mathcal{C}_{[1,2,1] \ell}$ semishort supermultiplets. For $A_{[0,6,0]}$ and $A_{[0,4,0]}$ using (6.8) we may show that this requires a non zero

$$
d_{\tau, \ell}^{[1,4,1]} \tau=1,2,3, \ell=1,3, \ldots, \quad d_{\tau, \ell}^{[0,4,0]} \tau=1,2,3,4, \ell=0,2, \ldots .
$$

where the cancellation of possible $\tau=-1,0$ terms depends on $b_{0, \ell}=-4 c_{1, \ell-1}$ and $a_{0, \ell+2}=$ $-4 c_{2, \ell}$. Here the $G_{\Delta}^{(\ell)}$ appearing in the final result for the OPE correspond to just those expected for operators belonging to $\mathcal{C}_{[0,4,0] \ell}, \mathcal{C}_{[1,2,1] \ell}$ semi-short supermultiplets. Detailed results for $d_{\tau, \ell}^{\mathcal{J}}$ are given in Appendix [F] It is critical that they are positive. 
A crucial test is whether all the remaining free field contributions can be represented as in (6.27). If we subtract off all contributions corresponding to long multiplets which gain anomalous dimensions we have in general

$$
A_{\mathcal{J}}^{\text {short }}=H_{\mathcal{J}}+F_{\mathcal{J}}\left(\mathcal{A}_{0}^{\text {short }}, \mathcal{B}_{0}^{\text {short }}, \mathcal{C}_{0}^{\text {short }}\right), \quad H_{\mathcal{J}}=A_{\mathcal{J}}^{\text {free }}-F_{\mathcal{J}}\left(\mathcal{A}_{0}, \mathcal{B}_{0}, \mathcal{C}_{0}\right)
$$

dropping any $1 / N^{2}$ terms in $A_{\mathcal{J}}^{\text {free }}$. Results for $H_{\mathcal{J}}(s, t)$ are given in Appendix $\mathbb{E}$ which are simple when expressed in terms of new variables $z, x$. Using this form it is straightforward to see that $H_{\mathcal{J}}$ corresponds to contributions which have only twist 2 or twist 0.

In detail we first consider $A_{[2,2,2]}^{\text {short }}$ and $A_{[3,0,3]}^{\text {short }}$. In this case only twist 2 contributions appear in the expansion of $H_{[2,2,2]}$ and $H_{[3,0,3]}$. Adding on the contributions resulting from $\mathcal{B}_{0}^{\text {short }}, \mathcal{C}_{0}^{\text {short }}$ for this case as in (6.32) there are again non trivial cancellations and we find non zero expansion coefficients just for

$$
d_{\tau, \ell}^{[2,2,2]} \tau=1,2,3, \ell=0,2, \ldots, \quad d_{\tau, \ell}^{[3,0,3]} \tau=1,2,3, \ell=1,3, \ldots .
$$

For $\mathcal{J}=[2,0,2],[0,2,0],[1,2,1],[1,0,1] H_{\mathcal{J}}$ contains both twist 0 and twist 2 but together with twist 4 such contributions cancel in $A_{\mathcal{J}}^{\text {short }}$ leaving

$$
d_{\tau, \ell}^{[2,0,2]}, d_{\tau, \ell}^{[0,2,0]} \tau=1,2,3,4, \ell=0,2, \ldots, \quad d_{\tau, \ell}^{[1,2,1]}, d_{\tau, \ell}^{[1,0,1]} \tau=1,2,3,4, \ell=1,3, \ldots
$$

to be non zero. The singlet case, $\mathcal{J}=[0,0,0]$ is further restricted in that there is cancellation of twist 6 terms as well leaving non zero

$$
d_{\tau, \ell}^{[0,0,0]} \tau=2,3,4, \ell=0,2, \ldots .
$$

It is then clear, for all representations $\mathcal{J}$, by matching (6.30), (6.31), (6.33), (6.34) and (6.35) with Table 1 that, to zeroth order in $1 / N$, that only operators belonging at least to the $\mathcal{C}_{[0,4,0] \ell}, \mathcal{C}_{[1,2,1] \ell}$ semi-short supermultiplets are necessary in the operator product expansion, although contributions in addition from $\mathcal{C}_{[2,0,2] \ell}$ are possible.

When $\ell=0,1$ then the results for $d_{\tau, \ell}^{\mathcal{J}}$ may be modified. First we note that we must have

$$
d_{-2,0}^{[0,0,0]}=50
$$

reflecting the contribution of the identity operator. For $\ell=0,1$ all $d_{\tau, \ell}^{\mathcal{J}}$ listed above are still present to zeroth order in $1 / N$ except for the twist 6 contribution

$$
d_{1,0}^{[0,2,0]}=0 .
$$

From Table 1 we may note that for twist 6 the operators in the $\mathcal{C}_{[0,4,0] 0}, \mathcal{C}_{[1,2,1] 1}$ semi-short multiplets in the $[0,2,0],[0,6,0],[2,0,2],[2,2,2]$ representations have lowest spin 2 while for $[1,0,1],[3,0,3]$ the lowest spin is 3 . Also for $\mathcal{C}_{[1,2,1] 1}$ we only have a twist $8[0,6,0]$ operator with spin 2 . From $\mathcal{C}_{[2,0,2] 0}$ we may have operators for $[2,0,2]$ with spin 0 and $[1,0,1],[3,0,3]$ with spin 1 . The remaining gaps correspond to operators which are part of short BPS multiplets. If we denote such multiplets by $\mathcal{B}_{\mathcal{J}}$, where if $\mathcal{J}=[q, p, q]$ with $q>0$ it is a $\frac{1}{4}$-BPS multiplet and if $q=0$ it is $\frac{1}{2}$-BPS, the relevant multiplets which may occur in the operator product expansion here with twist 6 or more are listed in Table 2 . 


\begin{tabular}{|c|c|c|c|c|c|c|c|c|}
\hline $\mathcal{B}_{[0,6,0]}$ & {$[0,2,0]$} & {$[0,4,0]$} & {$[2,2,2]$} & {$[0,6,0]$} & {$[1,2,1]$} & {$[1,4,1]$} & & \\
\hline 6 & & 2 & & 0 & & 1 & & \\
\hline 8 & & & 0 & & 1 & & & \\
\hline 10 & 0 & & & & & & & \\
\hline$\overline{\mathcal{B}}_{[2,2,2]}$ & $\overline{[\overline{[}[0,2,0]}$ & $\overline{[\overline{[0,4,0]}}$ & 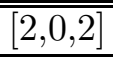 & 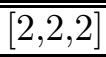 & 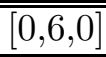 & $\overline{[1,0,1]}$ & {$[1,2,1]$} & {$[1,4,1]$} \\
\hline 6 & & 2 & 2 & 0,2 & & & 1,3 & 1 \\
\hline 8 & 0,2 & $\overline{0,2}$ & 0,2 & 0 & 0 & 1 & 1 & 1 \\
\hline 10 & 0 & 0 & 0 & 0 & & 1 & 1 & \\
\hline 12 & 0 & & & & & & & \\
\hline
\end{tabular}

Table 2: Spins of operators for given twist belonging to short BPS multiplets contributing to the operator product expansion of two $[0,3,0] \frac{1}{2}$-BPS multiplets with twist at least 6 .

Both BPS multiplets to account for operators which have been identified has necessarily present in the operator product expansion. It is evident that (6.37) is necessary for compatibility with the representation content of short and semi-short multiplets.

Thus we have shown that the OPE underlying the supergravity-induced four-point amplitude of $\frac{1}{2}$-BPS operators of dimension 3 fulfills the requirements of the superconformal symmetry and unitarity. Only those long multiplets belonging to the representations $[0,2,0]$, $[1,0,1]$ and $[0,0,0]$ may acquire possible anomalous scaling dimensions.

\section{Acknowledgements}

We are grateful to Sergei Frolov, Paul Heslop and Paul Howe for many useful discussions. E.S. wishes to thank the Max Planck Institute für Gravitationsphysik for the warm hospitality. G.A. was supported in part by the European Commission RTN programme HPRNCT-2000-00131 and by RFBI grant N02-01-00695.

\section{Appendices}

\section{A The $\mathcal{N}=2$ reduction formula}

Here we present a simplified version of the procedure of Ref. 18 for projecting an $\mathcal{N}=4$ four-point function of $\frac{1}{2}$-BPS operators onto the $\mathcal{N}=2$ hypermultiplet (HM) and SYM constituents. The new procedure can easily be applied to $\frac{1}{2}$-BPS operators of any dimension $k$. Further, we briefly recall the use of the $\mathcal{N}=2$ insertion formula from Ref. [19] and apply it to the correlator of weight $k=3$. In this way we can reproduce the results of Section 3 without reference to the $\mathcal{N}=4$ insertion formula.

The lowest component of the $\mathcal{N}=4$ field-strength multiplet $W^{i}, i=1, \ldots, 6$ is a real vector of $\mathrm{SO}(6)$. Reducing $\mathrm{SO}(6)$ to $\mathrm{SU}(3)$, we can decompose it into $3+\overline{3}$ :

$$
\mathcal{W}^{i} \rightarrow W^{A}, \bar{W}_{A}, \quad A=1,2,3 .
$$


The further decomposition $\mathrm{SU}(3) \rightarrow \mathrm{SU}(2) \times \mathrm{U}(1)$ results in

$$
W^{A} \rightarrow W^{a} \equiv \phi^{a}, a=1,2 ; \quad W^{3} \equiv w .
$$

After projection with $\mathrm{SU}(2)$ harmonics $\phi^{a}$ becomes the lowest component of the Grassmann analytic $\mathcal{N}=2 \mathrm{HM} q^{+}=u_{a}^{+} \phi^{a} ; w$ is the lowest component of the chiral $\mathcal{N}=2$ field strength; their conjugates are $\tilde{q}^{+}=u^{+a} \bar{\phi}_{a}$ and the antichiral $\bar{w}$.

The $\mathrm{SO}(6)$-covariant field-strength propagator is $\left\langle W^{i}(1) W^{j}(2)\right\rangle=\left\langle W^{j}(1) W^{i}(2)\right\rangle \sim \delta^{i j}$. Introducing $\mathrm{SO}(6)$ harmonics $1_{i}, 2_{i}$ and their symmetric contraction $(12)=(21)=1_{i} \delta^{i j} 2_{j}$, we can write the harmonic-projected $\mathrm{SO}(6)$ propagator $\langle W(1) W(2)\rangle \sim(12)$. Next, reducing $\mathrm{SO}(6)$ to $\mathrm{SU}(3)$ we decompose the $\mathrm{SO}(6)$ contraction into $\mathrm{SU}(3)$ pieces:

$$
(12)=1_{i} \delta^{i j} 2_{j}=1^{A} \overline{2}_{A}+\overline{1}_{A} 2^{A} \equiv[1 \overline{2}]+[\overline{1} 2] .
$$

In this notation we have "oriented" propagators for the $\mathrm{SU}(3)$-covariant field strengths: $\langle W \bar{W}\rangle=[1 \overline{2}]$ and $\langle\bar{W} W\rangle=[\overline{1} 2]$. The further reduction of $\mathrm{SU}(3)$ to $\mathrm{SU}(2) \times \mathrm{U}(1)$ gives, e.g., $[1 \overline{2}]=1^{A} \overline{2}_{A}=1^{a} \overline{2}_{a}+1^{3} \overline{2}_{3}$. This can be split into two independent propagators, one for the $\mathcal{N}=2 \mathrm{HM}$ :

$$
\langle q \tilde{q}\rangle \sim 1^{a} \overline{2}_{a}=1^{a} \epsilon_{a b} 2^{b}=-\overline{1}_{a} 2^{a} \equiv[12]=-[21]
$$

and one for the $\mathcal{N}=2$ field strength, $\langle w \bar{w}\rangle \sim 1^{3} \overline{2}_{3} \equiv 1$ (in the latter there is no need to use harmonics, $1^{3} \overline{2}_{3}$ is just a "bookkeeping device").

The $\frac{1}{2}$-BPS operator of weight $k$ is $\left.\operatorname{Tr}\left(\mathcal{W}^{\left\{i_{1}\right.} \ldots \mathcal{W}^{i_{k}}\right\}\right)$, where \{\} denotes traceless symmetrisation. Projected with $\mathrm{SO}(6)$ harmonic, it becomes $\mathcal{W}^{k}=\operatorname{Tr}\left(\mathcal{W}^{i_{1}} \cdots \mathcal{W}^{i_{k}}\right) 1_{i_{1}} \cdots 1_{i_{k}}$, and the absence of traces is guaranteed by the defining properties of the $\mathrm{SO}(6)$ harmonics. The four-point function for such operators has a harmonic structure consisting of all possible pairings of the four sets of harmonics. For instance, for $k=2$ we have

$$
\begin{aligned}
& \left\langle\mathcal{W}^{2}\left|\mathcal{W}^{2}\right| \mathcal{W}^{2} \mid \mathcal{W}^{2}\right\rangle \\
& =A_{1}(12)^{2}(34)^{2}+A_{2}(13)^{2}(24)^{2}+A_{3}(14)^{2}(23)^{2} \\
& \quad+B_{1}(13)(14)(23)(24)+B_{2}(12)(14)(23)(34)+B_{3}(12)(13)(24)(34),
\end{aligned}
$$

(compared to eq.(2.10), we have absorbed the space-time propagator factors into the coefficient functions $A, B, C)$.

The reduction to either $\mathcal{N}=2 \mathrm{HMs}$ or field strengths is straightforward. We replace each $\mathrm{SO}(6)$ contraction by $\mathrm{SU}(3)$ contractions: $(p q)=[p \bar{q}]+[\bar{p} q]$ and expand each $\mathrm{SO}(6)$ harmonic structure in (A.5) into products of $\mathrm{SU}(3)$ contractions. For example,

$$
\begin{aligned}
(12)^{2}(34)^{2} \rightarrow & {[\overline{1} 2]^{2}[\overline{3} 4]^{2}+2[\overline{1} 2]^{2}[\overline{3} 4][3 \overline{4}]+4[1 \overline{2}][\overline{1} 2][3 \overline{4}][\overline{3} 4]+\ldots } \\
(13)(14)(23)(24) \rightarrow & {[\overline{1} 3][\overline{1} 4][2 \overline{3}][2 \overline{4}]+[\overline{1} 3][\overline{1} 4][2 \overline{3}][2 \overline{4}] } \\
& +[1 \overline{3}][\overline{1} 4][\overline{2} 3][2 \overline{4}]+[\overline{1} 3][1 \overline{4}][2 \overline{3}][\overline{2} 4]+\ldots
\end{aligned}
$$

where we have displayed just the terms relevant for the two HM projections considered below. If we want to keep only the HM constituents of the composite operators, we need to replace the $\mathrm{SU}(3)$ contractions by $\mathrm{SU}(2)$ ones, taking care of the signs, e.g., $[1 \overline{2}] \rightarrow[12]$, $[\overline{1} 2] \rightarrow-[12]$. In this way we obtain, for example,

$$
\begin{aligned}
\left\langle\tilde{q}^{2}\left|q^{2}\right| \tilde{q}^{2} \mid q^{2}\right\rangle= & A_{1}[12]^{2}[34]^{2}+0[13]^{2}[24]^{2}+A_{3}[14]^{2}[23]^{2} \\
& +0[13][14][23][24]-B_{2}[12][14][23][34]+0[12][13][24][34] \\
\left\langle\tilde{q}^{2}\left|q^{2}\right| q \tilde{q} \mid q \tilde{q}\right\rangle= & -2 A_{1}[12]^{2}[34]^{2}+0[13]^{2}[24]^{2}+0[14]^{2}[23]^{2} \\
& +B_{1}[13][14][23][24]+B_{2}[12][14][23][34]-B_{3}[12][13][24][34] .
\end{aligned}
$$


Finally, with the help of the $\mathrm{SU}(2)$ harmonic cyclic identity [12][34] $+[13][42]+[14][23]=0$ we can eliminate, e.g., all factors of the type [13][24]. Thus, the projection (A.8) becomes

$$
\left\langle\tilde{q}^{2}\left|q^{2}\right| q \tilde{q} \mid q \tilde{q}\right\rangle=\left(-2 A_{1}-B_{3}\right)[12]^{2}[34]^{2}+B_{1}[14]^{2}[23]^{2}+\left(B_{1}+B_{2}-B_{3}\right)[12][34][14][23] .
$$

Similarly, to obtain the $\mathrm{U}(1)$ or chiral-antichiral $\mathcal{N}=2$ field-strength projection we replace every $(p q)$ in eq. A.5 by 1 if it corresponds to a Wick contraction of the type $\langle w \bar{w}\rangle$, or by 0 if it corresponds to $\langle w w\rangle$ or to $\langle\bar{w} \bar{w}\rangle$. In this way we find

$$
\left\langle w^{2}\left|\bar{w}^{2}\right| w^{2} \mid \bar{w}^{2}\right\rangle=A_{1}+A_{3}+B_{2} .
$$

Clearly, this procedure can easily be generalised to any dimension. In the case $k=3$ we have the decomposition (2.14) of the $\mathcal{N}=4$ amplitude into ten $\mathrm{SU}(4)$ harmonic structures. It has a large number of possible $\mathcal{N}=2 \mathrm{HM}$ projections, but it turns out that in order to derive the consequence of the $\mathcal{N}=2$ insertion formula it is sufficient to consider only one of them, $\left\langle q^{3}\left|\tilde{q}^{3}\right| q^{2} \tilde{q} \mid q \tilde{q}^{2}\right\rangle$. Repeating the steps described above, we easily obtain

$$
\begin{aligned}
\left\langle q^{3}\left|\tilde{q}^{3}\right| q^{2} \tilde{q} \mid q \tilde{q}^{2}\right\rangle= & \left(-3 A_{1}-B_{1}\right)[12]^{3}[34]^{3}-B_{3}[14]^{3}[23]^{3} \\
& +\left(-B_{1}+2 B_{2}+C\right)[12]^{2}[34]^{2}[14][23] \\
& +\left(-B_{3}-B_{4}+C\right)[14]^{2}[23]^{2}[12][34]
\end{aligned}
$$

In order to find the restrictions following from the insertion formula, we need not appeal to its $\mathcal{N}=4$ version, but can rely on the safer and well-understood $\mathcal{N}=2$ one based on the off-shell harmonic superspace formulation of the theory. The $\mathcal{N}=2$ insertion formula [15. 19] predicts that any $\mathcal{N}=2$ amplitude is a product of the dimension 2 polynomial

$$
R_{\mathcal{N}=2}^{2222}=\frac{[12]^{2}[34]^{2}}{x_{12}^{4} x_{34}^{4}} s+\frac{[14]^{2}[23]^{2}}{x_{14}^{4} x_{23}^{4}} t+\frac{[12][34][14][23]}{x_{12}^{2} x_{34}^{2} x_{14}^{2} x_{23}^{2}}(s+t-1)
$$

with another factor of dimension $k-2$ which contains arbitrary functions of $s, t$. In our case of dimension 3, for the projection in eq. A.11) this gives

$$
\left\langle q^{3}\left|\tilde{q}^{3}\right| q^{2} \tilde{q} \mid q \tilde{q}^{2}\right\rangle=R_{\mathcal{N}=2}^{2222} \quad\left[F(s, t) \frac{[12][34]}{x_{12}^{2} x_{34}^{2}}+G(s, t) \frac{[14][23]}{x_{14}^{2} x_{23}^{2}}\right] .
$$

Comparing the coefficients of each of the four independent harmonic structures in eqs. (A.11) and (A.13) we obtain four equations relating the coefficients $A, B, C$ to the newly introduced arbitrary functions $F, G$. Further, from the crossing symmetry relations (2.11), (2.15) and (2.16) it follows that only three of the coefficients $A, B, C$ are independent. Thus, the four equations impose a relation between the functions $F$ and $G$, so that in the end everything is expressed in terms of $G$. The independent function $\gamma(\underline{3.16})$ is related to $G$ as follows:

$$
\gamma=\frac{1}{3(t-s)}\left[\hat{G}-2 \tilde{\hat{G}}-\frac{1}{s}(\tilde{G}+\hat{\tilde{G}})\right]
$$

or inversely,

$$
G=\frac{t-s+1}{t} \hat{\gamma}-t \gamma
$$

Here $\tilde{G}(s, t)=G(t, s)$ and $\hat{G}(s, t)=G(s / t, 1 / t)$. In addition, the crossing symmetry condition $\gamma=\tilde{\gamma}$ is equivalent to the corresponding condition on $G$ in eq.(A.14):

$$
\left[\hat{G}+\frac{1}{s}(\tilde{G}+\hat{\tilde{G}})\right]+[\approx]=0 .
$$




\section{B C-algebra}

\section{B.1 $C$-tensors}

The supergravity fields of the five-dimensional effective action couple through $\mathrm{SO}(6)$ invariant tensors represented by overlapping integrals of spherical harmonics on the fivedimensional sphere:

$$
a_{123}=\int Y^{I_{1}} Y^{I_{2}} Y^{I_{3}}, \quad t_{123}=\int \nabla^{\alpha} Y^{I_{1}} Y^{I_{2}} Y_{\alpha}^{I_{3}}, \quad p_{123}=\int \nabla^{\alpha} Y^{I_{1}} \nabla^{\beta} Y^{I_{2}} Y_{(\alpha \beta)}^{I_{3}} .
$$

These are essentially the Clebsh-Gordon coefficients for the tensor product of $\mathrm{SO}(6)$ irreps, and it is in terms of these tensors that the cubic and quartic couplings of the effective action were expressed in Ref. [32].

The irreducible representations of $\mathrm{SO}(6)$ which are of interest to us here have Dynkin labels $[0, k, 0]$ and $[1, k-1,1]$ ( $k$ odd), $[2, k-2,2]$ ( $k$ even) and can be described in terms of the canonically normalised $C$-tensors with the corresponding Young symmetry. In particular, the irrep $[1, k, 1]$ is given by a tensor $C_{m ; i_{1} \ldots i_{k}}^{I}$ which is traceless symmetric w.r.t. $i_{1}, \ldots, i_{k}$ and has a vanishing symmetric part, while $[2, k, 2]$ is described by $C_{m n ; i_{1} \ldots i_{k}}^{I}$, traceless and symmetric w.r.t. $i_{1}, \ldots, i_{k}$ and $m, n$ separately, and obeying the constraint

$$
C_{m n ; i_{1} \ldots i_{k}}^{I}+C_{m i_{1} ; n \ldots i_{k}}^{I}+\ldots+C_{m i_{k} ; i_{1} \ldots n}^{I}=0 .
$$

We assume the following normalisations

$$
C_{m ; i_{1} \ldots i_{k}}^{I} C_{n ; i_{1} \ldots i_{k}}^{J}=\delta_{m n} \delta^{I J}, \quad C_{m_{1} n_{1} ; i_{1} \ldots i_{k}}^{I} C_{m_{2} n_{2} ; i_{1} \ldots i_{k}}^{J}=\delta^{I J} \delta_{m_{1} n_{1} ; m_{2} n_{2}} .
$$

The relation of the integrals of spherical harmonics to the $C$-tensors is as follows

$$
\begin{aligned}
a_{123} & =\frac{\prod_{i=1}^{3} \frac{k_{i} ! z\left(k_{i}\right)}{\alpha_{i} !}}{\pi^{\frac{3}{2}}(\sigma+2) ! 2^{\sigma-1}}\left\langle C_{\left[0, k_{1}, 0\right]}^{1} C_{\left[0, k_{2}, 0\right]}^{2} C_{\left[0, k_{3}, 0\right]}^{3}\right\rangle, \\
t_{123} & =\frac{\prod_{i=1}^{3} \frac{k_{i} ! z\left(k_{i}\right)}{\left(\alpha_{i}-\frac{1}{2}\right) !}}{\pi^{\frac{3}{2}}\left(k_{3}+1\right)\left(\sigma+\frac{3}{2}\right) ! 2^{\sigma-\frac{3}{2}}}\left\langle C_{\left[0, k_{1}, 0\right]}^{1} C_{\left[0, k_{2}, 0\right]}^{2} C_{\left[1, k_{3}-1,1\right]}^{3}\right\rangle, \\
p_{123} & =\frac{\alpha_{3} \prod_{i=1}^{3} \frac{k_{i} ! z\left(k_{i}\right)}{\alpha_{i} !}}{\pi^{\frac{3}{2}}(\sigma+1) ! 2^{\sigma}}\left\langle C_{\left[0, k_{1}, 0\right]}^{1} C_{\left[0, k_{2}, 0\right]}^{2} C_{\left[2, k_{3}-2,2\right]}^{3}\right\rangle,
\end{aligned}
$$

where $z(k)=\left(2^{k-1}(k+1)(k+2)\right)^{1 / 2}, \sigma=\frac{1}{2}\left(k_{1}+k_{2}+k_{3}\right)$ and $\alpha_{i}=\frac{1}{2}\left(k_{j}+k_{l}-k_{i}\right), j \neq l \neq i$. We use the notation $\left\langle C^{1} C^{2} C^{3}\right\rangle$ to denote the unique $\mathrm{SO}(6)$ tensor obtained by contracting particular subsets of indices of the three $C$-tensors $C_{[a, b, c]}^{I}$ (the subscript denotes the Dynkin labels of the corresponding irrep). Explicitly,

$$
\left\langle C_{\left[0, k_{1}, 0\right]}^{1} C_{\left[0, k_{2}, 0\right]}^{2} C_{\left[0, k_{3}, 0\right]}^{3}\right\rangle=C_{i_{1} \ldots i_{\alpha_{2}} j_{1} \ldots j_{\alpha_{3}}}^{I_{1}} C_{j_{1} \ldots j_{\alpha_{3}} l_{1} \ldots l_{\alpha_{1}}}^{I_{2}} C_{l_{1} \ldots l_{\alpha_{1}} i_{1} \ldots i_{\alpha_{2}}}^{I_{3}},
$$

and

$$
\begin{aligned}
\left\langle C_{\left[0, k_{1}, 0\right]}^{1} C_{\left[0, k_{2}, 0\right]}^{2} C_{\left[1, k_{3}-1,1\right]}^{3}\right\rangle= & C_{m i_{1} \ldots i_{p_{2} j_{1} \ldots j_{p_{3}}}}^{I_{1}} C_{j_{1} \ldots j_{p_{3}} l_{1} \ldots l_{p_{3}}}^{I_{2}} C_{m ; l_{1} \ldots l_{p_{1} i_{1} \ldots i_{p_{2}}}^{I_{3}}}^{I_{3}} \\
& -C_{i_{1} \ldots i_{p_{2}+1} j_{1} \ldots j_{p_{3}}}^{I_{1}} C_{j_{1} \ldots j_{p_{3}} l_{1} \ldots l_{p_{1}-1} m}^{I_{2}} C_{m ; l_{1} \ldots l_{p_{1}-1} i_{1} \ldots i_{p_{2}+1}}^{I_{3}}
\end{aligned}
$$


where $p_{1}=\alpha_{1}+\frac{1}{2}, p_{2}=\alpha_{2}-\frac{1}{2}$ and $p_{3}=\alpha_{3}-\frac{1}{2}$ and,

$$
\left\langle C_{\left[0, k_{1}, 0\right]}^{1} C_{\left[0, k_{2}, 0\right]}^{2} C_{\left[2, k_{3}-2,2\right]}^{3}\right\rangle=C_{m i_{1} \ldots i_{p_{2}} j_{1} \ldots j_{p_{3}}}^{I_{1}} C_{n j_{1} \ldots j_{p_{3}} l_{1} \ldots l_{p_{1}}}^{I_{2}} C_{m n ; l_{1} \ldots l_{p_{1} i_{1} \ldots i_{p_{2}}}^{I_{3}}}^{I_{3}} .
$$

Now we specialise these formulae to the case of interest when the legs 1 and 2 correspond to $k=3 \frac{1}{2}$-BPS operators, i.e. to the irrep $[0,3,0]$ (to simplify the notation, we will not display the Dynkin labels for these two legs). It is easy to see that $\left\langle C^{1} C^{2} C_{\left[0, k_{3}, 0\right]}^{3}\right\rangle \neq 0$ only if $k_{3}=0,2,4,6,\left\langle C^{1} C^{2} C_{\left[1, k_{3}-1,1\right]}^{3}\right\rangle \neq 0$ only if $k_{3}=1,3,5$ and $\left\langle C^{1} C^{2} C_{\left[2, k_{3}-2,2\right]}^{3}\right\rangle \neq 0$ only if $k_{3}=2,4$.

\section{B.2 Summation formulae}

The four-point function (2.21) is given in terms of ten independent tensor structures $\delta^{12} \delta^{34}$, $C^{1234}$ (and permutations) and $S^{1234}$. However, the AdS exchange graphs and the quartic couplings are expressed in terms of sums of the type

$$
\left\langle C^{1} C^{2} C^{5}\right\rangle\left\langle C^{3} C^{4} C^{5}\right\rangle,
$$

where summation over the representation index at the fifth point is assumed. Therefore we need to reexpress these sums in the basis of our ten independent tensor structures. This can be achieved by using the completeness condition for the C-tensors. Below we obtain the corresponding formulae.

In view of possible applications to the computation of correlation functions of higher $\frac{1}{2}$-BPS operators it is useful to derive a general formula for the sum $C_{i_{1} \ldots i_{n}}^{I} C_{j_{1} \ldots j_{n}}^{I}$ expressing the completeness condition. We have

$$
\left.C_{i_{1} \ldots i_{n}}^{I} C_{j_{1} \ldots j_{n}}^{I}=\sum_{k=0}^{\left[\frac{n}{2}\right]} \theta_{k} \sum_{\left(l_{1} \ldots l_{k}\right)} \delta_{i_{l_{1}} i_{l_{2}}} \ldots \delta_{i_{l_{k-1}} i_{l_{k}}} \delta^{(n-k)}\right)_{i_{1} \ldots \hat{i}_{l_{1}} \ldots \hat{i}_{l_{k}} \ldots i_{l_{n}},\left(j_{k+1} \ldots j_{n}\right.} \delta_{j_{1} j_{2}} \ldots \delta_{\left.j_{k-1} j_{k}\right)}
$$

Here $(\ldots)$ stands for total symmetrisation of indices, and $\delta^{(p)}{ }_{i_{1} \ldots i_{p}, j_{1} \ldots j_{p}}=\delta^{(p)}{ }_{\left(i_{1} \ldots i_{p}\right),\left(j_{1} \ldots j_{p}\right)}$ denotes the symmetrised product of $p$ Kronecker deltas $\delta_{i_{r} j_{s}}$. For every fixed $k$ the internal sum runs over all subsets $\left(l_{1} \ldots l_{k}\right) \in(1 \ldots n)$ which lead to different products

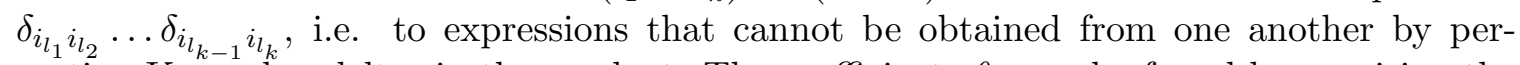
muting Kronecker deltas in the product. The coefficients $\theta_{k}$ can be found by requiring the r.h.s. of (B.8) to be traceless w.r.t. any pair of indices from $i_{1}, \ldots, i_{n}$ and they are

$$
\theta_{0}=1, \quad \theta_{k}=\frac{(-1)^{k}}{2^{k}(n+1) \ldots(n+2-k)}
$$

As an application of this formula we have, for instance,

$$
\begin{aligned}
C_{i_{1} i_{2} i_{3}}^{I} C_{j_{1} j_{2} j_{3}}^{I}=\frac{1}{6}\left[\delta_{i_{1} j_{1}} \delta_{i_{2} j_{2}} \delta_{i_{3} j_{3}}+\delta_{i_{1} j_{3}} \delta_{i_{2} j_{1}} \delta_{i_{3} j_{2}}+\delta_{i_{1} j_{2}} \delta_{i_{2} j_{3}} \delta_{i_{3} j_{1}}\right. \\
\left.+\delta_{i_{1} j_{2}} \delta_{i_{2} j_{1}} \delta_{i_{3} j_{3}}+\delta_{i_{1} j_{1}} \delta_{i_{2} j_{3}} \delta_{i_{3} j_{2}}+\delta_{i_{1} j_{3}} \delta_{i_{2} j_{2}} \delta_{i_{3} j_{1}}\right] \\
\quad-\frac{1}{8}\left[\delta_{i_{1} i_{2}} \delta_{i_{3}\left(j_{1}\right.} \delta_{\left.j_{2} j_{3}\right)}+\delta_{i_{1} i_{3}} \delta_{i_{2}\left(j_{1}\right.} \delta_{\left.j_{2} j_{3}\right)}+\delta_{i_{2} i_{3}} \delta_{i_{1}\left(j_{1}\right.} \delta_{\left.j_{2} j_{3}\right)}\right]
\end{aligned}
$$


Now substituting the completeness condition in the sum (B.7) with $C_{\left[0, k_{5}, 0\right]}^{5}$ and using the definitions (2.20), we obtain the following formulae (the case $k_{5}=0$ trivially gives $\left.\delta^{12} \delta^{34}\right)$

$$
\begin{aligned}
\left\langle C^{1} C^{2} C_{[0,2,0]}^{5}\right\rangle\left\langle C^{3} C^{4} C_{[0,2,0]}^{5}\right\rangle= & \frac{1}{2} C^{1234}+\frac{1}{2} C^{1243}-\frac{1}{6} \delta^{12} \delta^{34} \\
\left\langle C^{1} C^{2} C_{[0,4,0]}^{5}\right\rangle\left\langle C^{3} C^{4} C_{[0,4,0]}^{5}\right\rangle= & \frac{2}{3} S^{1234}+\frac{1}{6} C^{1324}+\frac{1}{6} C^{1423}-\frac{2}{15} C^{1234}-\frac{2}{15} C^{1243} \\
& +\frac{1}{60} \delta^{12} \delta^{34}, \\
\left\langle C^{1} C^{2} C_{[0,6,0]}^{5}\right\rangle\left\langle C^{3} C^{4} C_{[0,6,0]}^{5}\right\rangle= & \frac{1}{20}\left(\delta^{13} \delta^{24}+\delta^{14} \delta^{23}+9 C^{1342}+9 C^{1432}\right) \\
& -\frac{9}{140}\left(4 S^{1234}+C^{1324}+C^{1423}\right) \\
& +\frac{3}{140}\left(C^{1234}+C^{1243}\right)-\frac{1}{700} \delta^{12} \delta^{34} .
\end{aligned}
$$

The sum (B.7) with $C_{\left[1, k_{5}-1,1\right]}^{5}$ involves the tensors $C_{m ; i}^{I}, C_{m ; i j k}^{I}, C_{m ; i j k l p}^{I}$. The matrix $C_{m ; i}$ is antisymmetric and the corresponding completeness condition reads $C_{i ; j}^{I} C_{k ; l}^{I}=$ $\frac{1}{2}\left(\delta_{i k} \delta_{j l}-\delta_{i l} \delta_{j k}\right)$. Therefore, for $k_{5}=1$ it gives

$$
\left\langle C^{1} C^{2} C_{[1,0,1]}^{5}\right\rangle\left\langle C^{3} C^{4} C_{[1,0,1]}^{5}\right\rangle=2\left(C^{1234}-C^{1243}\right) .
$$

For the other two tensors the completeness conditions are more involved because of the mixed symmetry of the indices. Fortunately, there is another, indirect way to work out the corresponding sums. In [32] the following reduction relations were proved:

$$
\begin{aligned}
t_{125} t_{345}= & -\frac{\left(f_{1}-f_{2}\right)\left(f_{3}-f_{4}\right)}{4 f_{5}} a_{125} a_{345}+\frac{1}{4} f_{5}\left(a_{145} a_{235}-a_{245} a_{135}\right), \\
\left(1-f_{5}\right) t_{125} t_{345}= & \frac{1}{4}\left(f_{5}^{2}-f_{5}\left(f_{1}+f_{2}+f_{3}+f_{4}-4\right)\right)\left(a_{145} a_{235}-a_{135} a_{245}\right) \\
& -\frac{4-f_{5}}{4 f_{5}}\left(f_{1}-f_{2}\right)\left(f_{3}-f_{4}\right) a_{125} a_{345},
\end{aligned}
$$

where $f_{i}=k_{i}\left(k_{i}+4\right)$. Since we already know the sums (B.11) and their relation (B.1) to the integrals of the spherical harmonics, we can substitute them in the r.h.s. of (B.13). Further, using (B.12) and (B.2), we obtain two equations for the two remaining sums, whose solution is

$$
\begin{aligned}
\left\langle C^{1} C^{2} C_{[1,2,1]}^{5}\right\rangle\left\langle C^{3} C^{4} C_{[1,2,1]}^{5}\right\rangle= & \frac{1}{3}\left(-C^{1234}+C^{1243}+2 C^{1324}-2 C^{1423}\right), \\
\left\langle C^{1} C^{2} C_{[1,4,1]}^{5}\right\rangle\left\langle C^{3} C^{4} C_{[1,4,1]}^{5}\right\rangle= & \frac{1}{25}\left(C^{1234}-C^{1243}-5 C^{1324}+5 C^{1423}\right. \\
& \left.+15 C^{1342}-15 C^{1432}+5 \delta^{13} \delta^{24}-5 \delta^{14} \delta^{23}\right) .
\end{aligned}
$$

Analogously, using formulae (B.10) and (B.11) from [32] we obtain

$$
\left\langle C^{1} C^{2} C_{[2,0,2]}^{5}\right\rangle\left\langle C^{3} C^{4} C_{[2,0,2]}^{5}\right\rangle=-\frac{2}{15}\left(20 S^{1234}+5 C^{1234}+5 C^{1243}\right.
$$




$$
\begin{gathered}
\left.-10 C^{1324}-10 C^{1423}-\delta^{12} \delta^{34}\right) \\
\left\langle C^{1} C^{2} C_{[2,2,2]}^{5}\right\rangle\left\langle C^{3} C^{4} C_{[2,2,2]}^{5}\right\rangle=\frac{8}{15}\left(\frac{2}{7} C^{1234}+\frac{2}{7} C^{1243}-C^{1324}-C^{1342}-C^{1423}-C^{1432}\right. \\
\left.+\delta^{13} \delta^{24}+\delta^{14} \delta^{23}-\frac{1}{35} \delta^{12} \delta^{34}\right) .
\end{gathered}
$$

In conclusion, formulae (B.11), (B.14) and (B.15) are the necessary tools to express the contribution of the exchange graphs and the contact term in terms of the ten independent tensor structures.

\section{B.3 Pairings and normalised projectors}

Given the four-point function (2.14) one can analyze the underlying OPE. To this end one needs the (normalised) projectors on irreps appearing in the decomposition (2.13). This problem has already been partially solved since eqs.(B.11), (B.14) and (B.15) represent the (non-normalised) projectors on the corresponding irreps in (2.13). Only the projector on the irrep $[3,0,3]$ is missing. To normalize the projectors as well as to find the missing one, the pairings among the ten tensor structures of (2.14) have to be worked out. This is done by using the completeness relation (B.10) and the results are summarised in Table 3.

\begin{tabular}{c|ccccccc} 
Tensor & $C^{1234}$ & $C^{1243}$ & $C^{1324}$ & $C^{1342}$ & $C^{1423}$ & $C^{1432}$ & $S^{1234}$ \\
\hline$C^{1234}$ & $\frac{50375}{108}$ & $\frac{1375}{108}$ & $\frac{25525}{108}$ & $\frac{1025}{108}$ & $\frac{1025}{108}$ & $\frac{325}{108}$ & $\frac{575}{24}$ \\
$S^{1234}$ & $\frac{575}{24}$ & $\frac{575}{24}$ & $\frac{575}{24}$ & $\frac{575}{24}$ & $\frac{575}{24}$ & $\frac{575}{24}$ & $\frac{13225}{144}$ \\
$\delta^{12} \delta^{34}$ & $\frac{1250}{3}$ & $\frac{1250}{3}$ & 125 & $\frac{25}{3}$ & 125 & $\frac{25}{3}$ & $\frac{125}{3}$ \\
$\delta^{13} \delta^{24}$ & 125 & $\frac{25}{3}$ & $\frac{1250}{3}$ & $\frac{1250}{3}$ & $\frac{25}{3}$ & 125 & $\frac{125}{3}$ \\
$\delta^{14} \delta^{23}$ & $\frac{25}{3}$ & 125 & $\frac{25}{3}$ & 125 & $\frac{1250}{3}$ & $\frac{1250}{3}$ & $\frac{125}{3}$
\end{tabular}

Table 3: $C$-algebra. The number appearing at the intersection of a row and a column is the value of the pairing of the corresponding $C$-tensors, e.g. the value of $C^{1234} S^{1234}$ is $575 / 24$.

Using Table 3 we can easily find the expressions for the normalised projectors. Their normalisation is fixed to be $P_{\mathcal{J}}^{1234} P_{\mathcal{J}}^{1234}=\nu_{\mathcal{J}}$, where $\nu_{\mathcal{J}}$ is the dimension of the corresponding irrep. We find

$$
\begin{aligned}
P_{[0,0,0]}^{1234} & =\frac{1}{50} \delta^{12} \delta^{34}, \\
P_{[0,2,0]}^{1234} & =\frac{6}{35}\left(C^{1234}+C^{1243}-\frac{1}{3} \delta^{12} \delta^{34}\right),
\end{aligned}
$$




$$
\begin{aligned}
P_{[0,4,0]}^{1234}= & \frac{3}{7}\left(2 S^{1234}+\frac{1}{2} C^{1324}+\frac{1}{2} C^{1423}-\frac{2}{5} C^{1234}-\frac{2}{5} C^{1243}+\frac{1}{20} \delta^{12} \delta^{34}\right), \\
P_{[0,6,0]}^{1234}= & \frac{1}{20}\left(\delta^{13} \delta^{24}+\delta^{14} \delta^{23}+9 C^{1342}+9 C^{1432}\right. \\
\quad & \left.\quad-\frac{9}{7}\left(4 S^{1234}+C^{1324}+C^{1423}\right)+\frac{3}{7}\left(C^{1234}+C^{1243}\right)-\frac{1}{35} \delta^{12} \delta^{34}\right), \\
P_{[1,0,1]}^{1234}= & \frac{9}{70}\left(C^{1234}-C^{1243}\right), \\
P_{[1,2,1]}^{1234}= & \frac{1}{4}\left(-C^{1234}+C^{1243}+2 C^{1324}-2 C^{1423}\right), \\
P_{[1,4,1]}^{1234}= & \frac{1}{20}\left(C^{1234}-C^{1243}-5 C^{1324}+5 C^{1423}\right. \\
& \left.+15 C^{1342}-15 C^{1432}+5 \delta^{13} \delta^{24}-5 \delta^{14} \delta^{23}\right), \\
P_{[3,0,3]}^{1234}= & \frac{1}{28}\left(2 C^{1234}-2 C^{1243}-7 C^{1324}+7 C^{1423}-21 C^{1342}+21 C^{1432}\right. \\
\quad & \left.\quad+7 \delta^{13} \delta^{24}-7 \delta^{14} \delta^{23}\right), \\
P_{[2,0,2]}^{1234}= & \frac{3}{100}\left(-20 S^{1234}-5 C^{1234}-5 C^{1243}+10 C^{1324}+10 C^{1423}+\delta^{12} \delta^{34}\right), \\
P_{[2,2,2]}^{1234}= & \frac{9}{700}\left(-35 C^{1324}-35 C^{1342}-35 C^{1423}-35 C^{1432}\right.
\end{aligned}
$$

\section{Contact terms}

Here we extract the relevant contact interactions from the general quartic effective Lagrangian of 32. This Lagrangian can be written in the form

$$
\mathcal{L}_{4}=\mathcal{L}_{4}^{(4)} s^{1} \nabla_{\mu} s^{2} \nabla \cdot \nabla\left(s^{3} \nabla^{\mu} s^{4}\right)+\mathcal{L}_{4}^{(2)} s^{1} \nabla_{\mu} s^{2} s^{3} \nabla^{\mu} s^{4}+\mathcal{L}_{4}^{(0)} s^{1} s^{2} s^{3} s^{4},
$$

where $\mathcal{L}_{4}^{(4)}, \mathcal{L}_{4}^{(2)}$ and $\mathcal{L}_{4}^{(0)}$ are the corresponding couplings considered as functions of the representation labels $k_{1}=x, k_{2}=y, k_{3}=t, k_{4}=w$ of the four scalar fields $s^{I}$ involved (c.f. Appendix A of [32] $)^{6}$. For the case of interest we put $x=y=z=t=3$. Finally, in comparison with 32 we rescale the fields as $s \rightarrow \frac{\pi^{3 / 4}}{480^{1 / 2}} s$ to fit with our normalisations of the quadratic and the cubic terms in the actions of Section 4, and we change the overall sign to work with the Euclidean version of the AdS space.

\section{C.1 Four-derivative couplings}

First we sum up the quartic couplings of the four-derivative vertex

$\mathcal{L}_{4}^{(4)}=-\frac{\pi^{3}}{480^{2}}\left[\frac{1}{1024} f_{5}^{3}-\frac{7}{32} f_{5}^{2}+\frac{369}{32} f_{5}\right]\left(a_{145} a_{235}-a_{135} a_{245}\right)+\frac{\pi^{3}}{2^{18} \cdot 75}\left(f_{5}-1\right)^{2} t_{125} t_{345}$.

\footnotetext{
${ }^{6}$ One of the couplings in 32 was not printed out properly and we therefore reproduce it here $\left(S_{p 2}\right)_{I_{1} I_{2} I_{3} I_{4}}^{(0)}=\frac{2}{\delta} f_{5}^{2} p_{125} p_{345}\left(k_{1}^{2}+k_{2}^{2}+k_{3}^{2}+k_{4}^{2}-2\left(k_{1}+k_{2}+k_{3}+k_{4}\right)+2\left(k_{1} k_{2}+k_{3} k_{4}\right)-4\right)$.
} 
Next we express all the $a$ - and $t$-tensors in terms of $C$-tensors using formulae (B.1) and (B.2) and apply the summation formulae of Appendix B] As a result we get

$$
\mathcal{L}_{4}^{(4)}=\frac{3}{10240}\left(C^{1234}-C^{1243}+C^{1342}-C^{1432}+C^{1423}-C^{1324}\right) .
$$

On the other hand, for the $A d S_{5}$ background the four-derivative interaction can be written as

$$
s^{1} \nabla_{\mu} s^{2} \nabla \cdot \nabla\left(s^{3} \nabla^{\mu} s^{4}\right)=-10 s^{1} \nabla_{\mu} s^{2} s^{3} \nabla^{\mu} s^{4}+2 s^{1} \nabla_{\mu} s^{2} \nabla_{\nu} s^{3} \nabla^{\mu} \nabla^{\nu} s^{4} .
$$

The first term here is symmetric under $2 \leftrightarrow 4$ while the second term is symmetric under $2 \leftrightarrow 3$. These terms are further multiplied by the tensor (C.2), which is antisymmetric under $2 \leftrightarrow 4$ and independently under $2 \leftrightarrow 3$ and therefore the corresponding result vanishes. Hence there is no four-derivative contribution to the on-shell value of the gravity action.

\section{C.2 The remaining couplings}

Proceeding as before, i.e. expressing the total two-derivative coupling in terms of the $C$ tensors, we obtain

$$
\begin{aligned}
\mathcal{L}_{4}^{(2)}=-\frac{1}{1761607680} & {\left[3125461668 S^{1234}+323264681 C^{1234}+123826345 C^{1243}\right.} \\
& +826347689 C^{1324}-134041431 C^{1342}+733630633 C^{1423}+369041577 C^{1432} \\
& \left.-206335071 \delta^{12} \delta^{34}-53550175 \delta^{13} \delta^{24}+99234721 \delta^{14} \delta^{23}\right] .
\end{aligned}
$$

This expression looks rather ugly but this is in fact spurious because we have not yet taken into account that it is multiplied by $s^{1} \nabla_{\mu} s^{2} s^{3} \nabla^{\mu} s^{4}$, an expression which is symmetric w.r.t. $2 \leftrightarrow 4$. Indeed, with this in mind we see that eq. (C.3) is equivalent to

$$
\begin{aligned}
\mathcal{L}_{4}^{(2)} s^{1} \nabla_{\mu} s^{2} s^{3} \nabla^{\mu} s^{4}= & -\frac{1}{251658240}\left[446494524 S+98900894\left(C^{1234}+C^{1342}+C^{1423}\right)\right. \\
& \left.-7650025\left(\delta^{12} \delta^{34}+\delta^{14} \delta^{23}+\delta^{13} \delta^{24}\right)\right] s^{1} \nabla_{\mu} s^{2} s^{3} \nabla^{\mu} s^{4} \\
& -\frac{3}{32} C^{1243} s^{1} \nabla_{\mu} s^{2} s^{3} \nabla^{\mu} s^{4} .
\end{aligned}
$$

It is clear that the tensor in the square brackets is totally symmetric under permutations of the indices $(2,3,4)$.

Now, consider the expression

$$
I=\chi^{1234} s^{1} \nabla_{\mu} s^{2} s^{3} \nabla^{\mu} s^{4},
$$

where $\chi^{1234}$ is a totally symmetric tensor. Let us show that such an interaction term can be reduced to a term without derivatives. Indeed, under integration by parts we obtain

$$
\begin{aligned}
\chi^{1234} s^{1} \nabla_{\mu} s^{2} s^{3} \nabla^{\mu} s^{4}= & \chi^{1234} s^{1} \nabla_{\mu} s^{3} s^{2} \nabla^{\mu} s^{4}=-\chi^{1234} \nabla_{\mu} s^{1} s^{2} s^{3} \nabla^{\mu} s^{4}-\chi^{1234} s^{1} s^{3} \nabla_{\mu} s^{2} \nabla^{\mu} s^{4} \\
& -m_{4}^{2} \chi^{1234} s^{1} s^{2} s^{3} s^{4} .
\end{aligned}
$$

Hence we find

$$
\chi^{1234} s^{1} \nabla_{\mu} s^{2} s^{3} \nabla^{\mu} s^{4}=-\frac{m_{4}^{2}}{3} \chi^{1234} s^{1} s^{2} s^{3} s^{4}=\chi^{1234} s^{1} s^{2} s^{3} s^{4},
$$


where we have used the fact that $m_{4}^{2}=-3$. Therefore we can use this trick to reduce the totally symmetric part of the term $\mathcal{L}_{4}^{(2)} s^{1} \nabla_{\mu} s^{2} s^{3} \nabla^{\mu} s^{4}$ to a term without derivatives.

Next, the coupling without derivatives reads

$$
\mathcal{L}_{4}^{(0)}=\frac{68665157}{20971520} S^{1234}+\frac{100568527}{41943040} C^{1234}-\frac{4675733}{16777216} \delta^{12} \delta^{34},
$$

where we have taken into account that this coupling is multiplied by $s^{1} s^{2} s^{3} s^{4}$ which is symmetric under permutations of $2,3,4$.

Finally, summing up $\mathcal{L}_{4}^{(0)}$ with the non-derivative term obtained from the symmetric part of $\mathcal{L}_{4}^{(2)}$, we obtain the spectacularly simple final expression in eq.(4.4).

\section{D-functions}

\section{D.1 D-operators}

Here we collect the necessary facts about the $D$-functions. The $D$-functions related to $A d S_{d+1}$ are defined by the formula

$$
D_{\Delta_{1} \Delta_{2} \Delta_{3} \Delta_{4}}\left(x_{1}, x_{2}, x_{3}, x_{4}\right)=\int \frac{\mathrm{d}^{d} w \mathrm{~d} w_{0}}{w_{0}^{d+1}} \prod_{i=1}^{4} K_{\Delta_{i}}\left(w, x_{i}\right)
$$

for

$$
K_{\Delta}(w, x)=\left(\frac{w_{0}}{w_{0}^{2}+(\vec{w}-x)^{2}}\right)^{\Delta},
$$

and where the integral is taken over the space parametrised by $w_{\mu}=\left(w_{0}, \vec{w}\right), \vec{w}$ being a $d$-dimensional vector, $w_{0} \geq 0$. From this definition one can deduce the following Feynman parameter representation

$$
D_{\Delta_{1} \Delta_{2} \Delta_{3} \Delta_{4}}\left(x_{1}, x_{2}, x_{3}, x_{4}\right)=\frac{\pi^{\frac{1}{2} d} \Gamma\left(\Sigma-\frac{d}{2}\right) \Gamma(\Sigma)}{2 \prod_{i} \Gamma\left(\Delta_{i}\right)} \int \prod_{j} \mathrm{~d} \alpha_{j} \alpha_{j}^{\Delta_{j}-1} \frac{\delta\left(\sum_{j} \alpha_{j}-1\right)}{\left(\sum_{k<l} \alpha_{k} \alpha_{l} x_{k l}^{2}\right)^{\Sigma}},
$$

where $\Sigma=\frac{1}{2} \sum_{i=1}^{4} \Delta_{i}$. From the $D$ functions we may define corresponding functions of the conformal invariants $s, t$ by

$$
\frac{\prod_{i=1}^{4} \Gamma\left(\Delta_{i}\right)}{\Gamma\left(\Sigma-\frac{1}{2} d\right)} \frac{2}{\pi^{\frac{1}{2} d}} D_{\Delta_{1} \Delta_{2} \Delta_{3} \Delta_{4}}\left(x_{1}, x_{2}, x_{3}, x_{4}\right)=\frac{\left(x_{14}^{2}\right)^{\Sigma-\Delta_{1}-\Delta_{4}}\left(x_{34}^{2}\right)^{\Sigma-\Delta_{3}-\Delta_{4}}}{\left(x_{13}^{2}\right)^{\Sigma-\Delta_{4}}\left(x_{24}^{2}\right)^{\Delta_{2}}} \bar{D}_{\Delta_{1} \Delta_{2} \Delta_{3} \Delta_{4}}(s, t) .
$$

For $\Delta_{i}=1$ we have

$$
\bar{D}_{1111}(s, t)=\Phi(s, t),
$$

where $\Phi(s, t)$ given in terms of the standard four-dimensional one-loop (box) integral considered as a function of the conformal cross-ratios $s$ and $t$. It has an explicit representation in terms of dilogarithms [43.

The $D$-functions satisfy the following derivative relation

$$
\frac{\partial}{\partial x_{12}^{2}} D_{\Delta_{1} \Delta_{2} \Delta_{3} \Delta_{4}}=\frac{\Delta_{1} \Delta_{2}}{\frac{1}{2} d-\Sigma} D_{\Delta_{1}+1 \Delta_{2}+1 \Delta_{3} \Delta_{4}}
$$


and similarly for any other pair of indices which leads to

$$
\begin{aligned}
& \bar{D}_{\Delta_{1}+1 \Delta_{2}+1 \Delta_{3} \Delta_{4}}=-\partial_{s} \bar{D}_{\Delta_{1} \Delta_{2} \Delta_{3} \Delta_{4}}, \\
& \bar{D}_{\Delta_{1} \Delta_{2} \Delta_{3}+1 \Delta_{4}+1}=\left(\Delta_{3}+\Delta_{4}-\Sigma-s \partial_{s}\right) \bar{D}_{\Delta_{1} \Delta_{2} \Delta_{3} \Delta_{4}}, \\
& \bar{D}_{\Delta_{1} \Delta_{2}+1 \Delta_{3}+1 \Delta_{4}}=-\partial_{t} \bar{D}_{\Delta_{1} \Delta_{2} \Delta_{3} \Delta_{4}}, \\
& \bar{D}_{\Delta_{1}+1 \Delta_{2} \Delta_{3} \Delta_{4}+1}=\left(\Delta_{1}+\Delta_{4}-\Sigma-t \partial_{t}\right) \bar{D}_{\Delta_{1} \Delta_{2} \Delta_{3} \Delta_{4}}, \\
& \bar{D}_{\Delta_{1} \Delta_{2}+1 \Delta_{3} \Delta_{4}+1}=\left(\Delta_{2}+s \partial_{s}+t \partial_{t}\right) \bar{D}_{\Delta_{1} \Delta_{2} \Delta_{3} \Delta_{4}}, \\
& \bar{D}_{\Delta_{1}+1 \Delta_{2} \Delta_{3}+1 \Delta_{4}}=\left(\Sigma-\Delta_{4}+s \partial_{s}+t \partial_{t}\right) \bar{D}_{\Delta_{1} \Delta_{2} \Delta_{3} \Delta_{4}} .
\end{aligned}
$$

This allows differential operators $\overline{\mathbf{D}}_{\Delta_{1} \Delta_{2} \Delta_{3} \Delta_{4}}$ to be obtained so that (5.2) is valid whenever $\Delta_{i}, \Sigma$ are integers. The differential operators are not unique, clearly $\bar{D}_{2222}$ can be obtained by using three separate pairs of equations in (D.6) giving the relations

$$
\partial_{s} s \partial_{s} \Phi=\partial_{t} t \partial_{t} \Phi=\left(s \partial_{s}+t \partial_{t}+1\right)^{2} \Phi
$$

The action of the derivatives on $\Phi$ is given by [19]

$$
\begin{aligned}
& \partial_{s} \Phi(s, t)=\frac{1}{\lambda^{2}}\left(\Phi(s, t)(1-s+t)+2 \ln s-\frac{s+t-1}{s} \ln t\right), \\
& \partial_{t} \Phi(s, t)=\frac{1}{\lambda^{2}}\left(\Phi(s, t)(1-t+s)+2 \ln t-\frac{s+t-1}{t} \ln s\right),
\end{aligned}
$$

where $\lambda=\sqrt{(1-s-t)^{2}-4 s t}$.

Using (D.6) we can obtain expressions for all the $\bar{D}$-functions we are interested in as differential operators acting on $\Phi(s, t)$, up to the arbitrariness following from (D.7). In particular, the following $\overline{\mathbf{D}}$-operators

$$
\begin{array}{ll}
\overline{\mathbf{D}}_{3311}=\partial_{s}{ }^{2} & \overline{\mathbf{D}}_{3232}=\left(1+s \partial_{s}\right)\left(2+s \partial_{s}+t \partial_{t}\right) \partial_{s} \\
\overline{\mathbf{D}}_{3322}=-\left(2+s \partial_{s}\right) \partial_{s}^{2} & \overline{\mathbf{D}}_{3333}=-\left(1+s \partial_{s}\right)\left(2+s \partial_{s}+t \partial_{t}\right)^{2} \\
\overline{\mathbf{D}}_{2332}=-\left(1+s \partial_{s}\right) \partial_{t} \partial_{s} & \overline{\mathbf{D}}_{3412}=\left(3+s \partial_{s}+t \partial_{t}\right) \partial_{s}{ }^{2} \\
\overline{\mathbf{D}}_{3421}=-\partial_{t} \partial_{s}^{2} & \overline{\mathbf{D}}_{3243}=-\left(1+s \partial_{s}\right)\left(2+s \partial_{s}+t \partial_{t}\right) s \partial_{s}{ }^{2} \\
\overline{\mathbf{D}}_{3423}=-\left(2+s \partial_{s}\right)\left(3+s \partial_{s}+t \partial_{t}\right) \partial_{s}^{2} & \overline{\mathbf{D}}_{3342}=-\partial_{t}\left(1+s \partial_{s}\right)\left(2+s \partial_{s}+t \partial_{t}\right) \partial_{s} \\
\overline{\mathbf{D}}_{3432}=\partial_{t}\left(2+s \partial_{s}\right)\left(3+s \partial_{s}+t \partial_{t}\right) \partial_{s}^{2} & \overline{\mathbf{D}}_{4334}=-t \partial_{t}\left(1+s \partial_{s}\right)\left(2+s \partial_{s}+t \partial_{t}\right) \partial_{s} \\
\overline{\mathbf{D}}_{4433}=-\partial_{s}\left(1+s \partial_{s}\right)\left(2+s \partial_{s}+t \partial_{t}\right) \partial_{s} &
\end{array}
$$

may be used to determine the coefficient functions of Section 5 .

\section{D.2 Relations for $\bar{D}$-functions and simplification of the supergravity am- plitude}

Here we show how the original coefficient functions (5.1) can be further simplified and present an independent proof of the splitting property of the supergravity-induced fourpoint amplitude into "free" and "quantum" parts.

By virtue of the obvious permutation symmetries in (D.1) we have

$$
\bar{D}_{\Delta_{1} \Delta_{2} \Delta_{3} \Delta_{4}}(s, t)=t^{-\Delta_{2}} \bar{D}_{\Delta_{1} \Delta_{2} \Delta_{4} \Delta_{3}}(s / t, 1 / t)
$$




$$
\begin{aligned}
& =t^{\Delta_{4}-\Sigma} \bar{D}_{\Delta_{2} \Delta_{1} \Delta_{3} \Delta_{4}}(s / t, 1 / t) \\
& =\bar{D}_{\Delta_{3} \Delta_{2} \Delta_{1} \Delta_{4}}(t, s) \\
& =t^{\Delta_{1}+\Delta_{4}-\Sigma} \bar{D}_{\Delta_{2} \Delta_{1} \Delta_{4} \Delta_{3}}(s, t) \\
& =s^{\Delta_{3}+\Delta_{4}-\Sigma} \bar{D}_{\Delta_{4} \Delta_{3} \Delta_{2} \Delta_{1}}(s, t) .
\end{aligned}
$$

We also have the reflection property

$$
\bar{D}_{\Delta_{1} \Delta_{2} \Delta_{3} \Delta_{4}}(s, t)=\bar{D}_{\Sigma-\Delta_{3} \Sigma-\Delta_{4} \Sigma-\Delta_{1} \Sigma-\Delta_{2}}(s, t) .
$$

In addition there are relations involving $\bar{D}$ functions with differing $\Sigma$. First there are what may be referred to as the two up, two down relations

$$
\begin{aligned}
& \left(\Delta_{2}+\Delta_{4}-\Sigma\right) \bar{D}_{\Delta_{1} \Delta_{2} \Delta_{3} \Delta_{4}}(s, t)=\bar{D}_{\Delta_{1} \Delta_{2}+1 \Delta_{3} \Delta_{4}+1}(s, t)-\bar{D}_{\Delta_{1}+1 \Delta_{2} \Delta_{3}+1 \Delta_{4}}(s, t) \\
& \left(\Delta_{1}+\Delta_{4}-\Sigma\right) \bar{D}_{\Delta_{1} \Delta_{2} \Delta_{3} \Delta_{4}}(s, t)=\bar{D}_{\Delta_{1}+1 \Delta_{2} \Delta_{3} \Delta_{4}+1}(s, t)-t \bar{D}_{\Delta_{1} \Delta_{2}+1 \Delta_{3}+1 \Delta_{4}}(s, t) \\
& \left(\Delta_{3}+\Delta_{4}-\Sigma\right) \bar{D}_{\Delta_{1} \Delta_{2} \Delta_{3} \Delta_{4}}(s, t)=\bar{D}_{\Delta_{1} \Delta_{2} \Delta_{3}+1 \Delta_{4}+1}(s, t)-s \bar{D}_{\Delta_{1}+1 \Delta_{2}+1 \Delta_{3} \Delta_{4}}(s, t) .
\end{aligned}
$$

and then the following formula involving the sum of three $\bar{D}$ functions with the same $\Sigma$

$$
\begin{aligned}
\Delta_{4} \bar{D}_{\Delta_{1} \Delta_{2} \Delta_{3} \Delta_{4}}(s, t)= & \bar{D}_{\Delta_{1} \Delta_{2} \Delta_{3}+1 \Delta_{4}+1}(s, t)+\bar{D}_{\Delta_{1} \Delta_{2}+1 \Delta_{3} \Delta_{4}+1}(s, t) \\
& +\bar{D}_{\Delta_{1}+1 \Delta_{2} \Delta_{3} \Delta_{4}+1}(s, t) .
\end{aligned}
$$

These results give relations between $\bar{D}_{\Delta_{1} \Delta_{2} \Delta_{3} \Delta_{4}}$ for any fixed $\Sigma$ and are necessary for consistency of (D.6). If $\Delta_{i}=n_{i}$ for integers $n_{i}$ then for any $\sum_{i} n_{i}=2 \Sigma$ even all $\bar{D}$-functions may be related ${ }^{7}$.

For one $\Delta_{i}=0$ the integral (D.1) reduces to a three point function. Using the above this leads to

$$
\begin{aligned}
& \left.\left(\bar{D}_{\Delta_{1}+1 \Delta_{2} \Delta_{3}+1 \Delta_{4}}+s \bar{D}_{\Delta_{1}+1 \Delta_{2}+1 \Delta_{3} \Delta_{4}}+t \bar{D}_{\Delta_{1} \Delta_{2}+1 \Delta_{3}+1 \Delta_{4}}\right)\right|_{\Delta_{4}=\Delta_{1}+\Delta_{2}+\Delta_{3}} \\
& \quad=\Gamma\left(\Delta_{1}\right) \Gamma\left(\Delta_{2}\right) \Gamma\left(\Delta_{3}\right) .
\end{aligned}
$$

This leads, for $\Delta_{1}=\Delta_{2}=\Delta_{3}=1$, to the following inhomogeneous differential equation for $\Phi$

$$
\left[(1-s-t) \partial_{s} \partial_{t}-\partial_{s}^{2} s-\partial_{t}^{2} t\right] \Phi(s, t)=\frac{1}{s t} .
$$

Both (D.7) and (D.14) follow from (D.8).

These results may now be used to simplify the results of the supergravity calculations. For simplicity let

$$
\left(a_{i}, b_{i}, c, \alpha, \beta, \gamma\right)=\frac{9}{2 N^{2}}\left(\hat{a}_{i}, \hat{b}_{i}, \hat{c}, \hat{\alpha}, \hat{\beta}, \hat{\gamma}\right)
$$

\footnotetext{
${ }^{7}$ Let $\left(n_{1}, n_{2}, n_{3}, n_{4}\right)$ be the set of integers corresponding to a $\bar{D}$ function. Let $\left(n_{1}, n_{2}, n_{3}, n_{4}\right) \sim$ $\left(n_{1}^{\prime}, n_{2}^{\prime}, n_{3}^{\prime}, n_{4}^{\prime}\right)$ if the $\bar{D}$ function is related up to contributions with lower $\Sigma$. From (D.11) $\left(n_{1}, n_{2}, n_{3}, n_{4}\right) \sim$ $\left(n_{1}+1, n_{2}-1, n_{3}+1, n_{4}-1\right) \sim\left(n_{1}+2, n_{2}-2, n_{3}, n_{4}\right)$ and thus we may reduce $\left(n_{1}, n_{2}\right)$ to one of the form $(m+2, m),(m+1, m),(m, m)$ and similarly $\left(n_{3}, n_{4}\right)$ to $(n+2, n),(n+1, n),(n, n)$. The relation (D.11) then allows $m \rightarrow m \pm 1$ while $n=n \mp 1$ so that we can take $m=n+1$ or $m=n$. There are then 10 possibilities but these can be reduced to $(n+3, n+1, n+2, n) \sim(n+2, n+2, n+1, n+1)$, $(n+2, n, n+2, n) \sim(n+1, n+1, n+1, n+1),(n+1, n+1, n+2, n)$ and $(n, n, n+2, n)$. Using (D.12) $(n, n, n+2, n) \sim-(n+1, n, n+1, n)-(n, n+1, n+1, n)$. Similarly $(n+1, n+1, n+2, n)$ can be reduced to $(n+1, n+1, n+1, n+1)$.
} 
and then we may write from (5.1)

$$
\hat{a}_{1}=s^{3}\left((1+t-s)\left(\bar{D}_{4433}+\bar{D}_{4422}\right)+5 \bar{D}_{3333}-\bar{D}_{3311}\right) .
$$

To simplify this we use (D.11) and (D.12) three times in each case to obtain

$$
\begin{aligned}
& (1+t-s) \bar{D}_{4433}=-2 \bar{D}_{4424}+2\left(\bar{D}_{4323}+\bar{D}_{3423}\right)-3 \bar{D}_{3333} \\
& (1+t-s) \bar{D}_{4422}=2 \bar{D}_{4413}+\bar{D}_{4312}+\bar{D}_{3412}-3 \bar{D}_{3322}
\end{aligned}
$$

Inserting this in (D.16) and using (D.12) again gives

$$
\hat{a}_{1}=-2 s^{3}\left(\bar{D}_{4424}+\bar{D}_{4413}\right) .
$$

With further use of (D.9) and (D.10) we obtain

$$
\begin{aligned}
& \hat{\alpha}=-2\left(s \bar{D}_{3335}+\bar{D}_{2235}\right)=-2 s^{2}\left(\bar{D}_{5333}+\bar{D}_{5322}\right), \\
& \hat{\beta}=-2\left(t \bar{D}_{3335}+\bar{D}_{3225}\right)=-2\left(\bar{D}_{4226}-\bar{D}_{3225}\right) \\
& \hat{\gamma}=-2\left(\bar{D}_{3335}+\bar{D}_{2325}\right)=-2 s\left(\bar{D}_{5333}+\bar{D}_{5232}\right) .
\end{aligned}
$$

For further simplification we first consider $b_{1}$ where we have from (5.1),

$$
\begin{aligned}
\hat{b}_{1}=s^{2}\left((1+t-s) \bar{D}_{4433}-4 \bar{D}_{4334}-4 \bar{D}_{3243}+4 t \bar{D}_{3342}-\bar{D}_{3423}+t \bar{D}_{3432}\right. \\
\left.\quad+8 \bar{D}_{3232}-4 \bar{D}_{3322}-2 \bar{D}_{3412}+2 t \bar{D}_{3421}+2 \bar{D}_{3311}\right) \\
=s^{2}\left(-2 \bar{D}_{4424}-4 \bar{D}_{4334}+2\left(\bar{D}_{4323}+\bar{D}_{3423}\right)-3 \bar{D}_{3333}-4 \bar{D}_{3243}+4 \bar{D}_{4233}\right. \\
\left.\quad-\bar{D}_{3423}+\bar{D}_{4323}+8 \bar{D}_{3232}-4 \bar{D}_{3322}-2 \bar{D}_{3412}+2 \bar{D}_{4312}+2 \bar{D}_{3311}\right) .
\end{aligned}
$$

According to the general results obtained in Section 3 this is related to $(t-s-1) \hat{\alpha}+s \hat{\gamma}$. From (D.19) we need

$$
\begin{aligned}
& (t-s-1) \bar{D}_{5333}=2 \bar{D}_{4334}+\bar{D}_{5223}-3\left(\bar{D}_{4233}+\bar{D}_{4323}\right) \\
& (t-s-1) \bar{D}_{5322}=2 \bar{D}_{4323}-3 \bar{D}_{4222}-2 \bar{D}_{4312}+\frac{1}{s^{2}}
\end{aligned}
$$

where the last result depends on using (D.13). Hence we obtain

$$
\begin{aligned}
(t-s-1) \hat{\alpha}+s \hat{\gamma}= & s^{2}\left(-4 \bar{D}_{4334}-2 \bar{D}_{5333}-2 \bar{D}_{5223}+6\left(\bar{D}_{4233}+\bar{D}_{4323}\right)\right. \\
& \left.-2 \bar{D}_{5232}-4 \bar{D}_{4323}+6 \bar{D}_{4222}+4 \bar{D}_{4312}\right)-2
\end{aligned}
$$

Combining (D.20) and (D.22) we readily find

$$
\hat{b}_{1}=(t-s-1) \hat{\alpha}+s \hat{\gamma}+2 .
$$

The results for $c$ can be similarly simplified. The starting point is

$$
\hat{c}=32 s t\left(-\frac{3}{2} \bar{D}_{3333}+\bar{D}_{2332}+\bar{D}_{3232}+\bar{D}_{3322}\right) .
$$


For comparison we have

$$
\begin{aligned}
(s- & t-1) \hat{\alpha}+(t-s-1) \hat{\beta}+(1-s-t) \hat{\gamma} \\
= & -4 s \bar{D}_{3326}-4 t \bar{D}_{2336}-4 \bar{D}_{3236} \\
& -8(s-t-1) \bar{D}_{2235}-8(t-s-1) \bar{D}_{3225}-8(1-s-t) \bar{D}_{2325} \\
= & -4+8 s t\left(-\bar{D}_{2253}-\bar{D}_{5223}+\bar{D}_{3252}+\bar{D}_{5232}-\bar{D}_{2523}\right) \\
& +8 t\left(\bar{D}_{2235}+\bar{D}_{2253}\right)+8 s\left(\bar{D}_{3225}+\bar{D}_{5223}\right),
\end{aligned}
$$

where we have used (D.13). Using (D.9) and (D.11) to generate an overall factor of st we may now use (D.12) to demonstrate

$$
\hat{c}=(s-t-1) \hat{\alpha}+(t-s-1) \hat{\beta}+(1-s-t) \hat{\gamma}+4 .
$$

(D.23) and (D.26) are equivalent to (5.4).

For determining anomalous dimensions we need to identify the terms in $\bar{D}_{\Delta_{1} \Delta_{2} \Delta_{3} \Delta_{4}}(s, t)$ involving $\ln s$ in an expansion in terms of $s, 1-t$. For $N=\Delta_{1}+\Delta_{2}-\Sigma+1=1,2, \ldots$ we have from [4]

$$
\begin{aligned}
\bar{D}_{\Delta_{1} \Delta_{2} \Delta_{3} \Delta_{4}}(s, t) \sim & \ln s \frac{(-1)^{N}}{(N-1) !} \frac{\Gamma\left(\Delta_{1}\right) \Gamma\left(\Delta_{2}\right) \Gamma\left(\Sigma-\Delta_{3}\right) \Gamma\left(\Sigma-\Delta_{4}\right)}{\Gamma\left(\Delta_{1}+\Delta_{2}\right)} \\
& \times \sum_{m, n=0}^{\infty} \frac{\left(\Delta_{1}\right)_{m}\left(\Sigma-\Delta_{3}\right)_{m}\left(\Delta_{2}\right)_{m+n}\left(\Sigma-\Delta_{4}\right)_{m+n}}{m ! n !(N)_{m}\left(\Delta_{1}+\Delta_{2}\right)_{2 m+n}} s^{m}(1-t)^{n},
\end{aligned}
$$

where $(x)_{m}=\Gamma(x+m) / \Gamma(x)$. For $N=2,3, \ldots$ there are additional terms which dominate for $s \sim 0$ and which do not contain $\ln s$,

$$
\begin{aligned}
& s^{1-N}(N-2) ! \frac{\Gamma\left(\Delta_{1}-N+1\right) \Gamma\left(\Delta_{2}-N+1\right) \Gamma\left(\Sigma-\Delta_{3}-N+1\right) \Gamma\left(\Sigma-\Delta_{4}-N+1\right)}{\Gamma\left(\Delta_{1}+\Delta_{2}-2 N+2\right)} \\
& \times \sum_{m=0}^{N-2} \sum_{n=0}^{\infty} \frac{\left(\Delta_{1}-N+1\right)_{m}\left(\Sigma-\Delta_{3}-N+1\right)_{m}\left(\Delta_{2}-N+1\right)_{m+n}\left(\Sigma-\Delta_{4}-N+1\right)_{m+n}}{m ! n !(1-N)_{m}\left(\Delta_{1}+\Delta_{2}-2 N+2\right)_{2 m+n}} s^{m}(1-t)^{n} .
\end{aligned}
$$

\section{E Exchange graphs of a massive symmetric tensor}

Here we show how the method of Ref. [36] can be generalised to compute the AdS graphs involving the exchange of a massive symmetric tensor.

Recall that an exchange graph is a double integral in the $z$ and $w$ variables over the fivedimensional AdS space. One first computes the $z$-integral and then recasts the remaining $w$-integral as a sum of different $D$-functions.

We start with the equations of motion for the massive symmetric tensor $\varphi_{\mu \nu}$. The Lagrangian is given by 32

$$
\begin{aligned}
\mathcal{L}= & -\frac{1}{4} \nabla_{\rho} \varphi_{\mu \nu} \nabla^{\rho} \varphi^{\mu \nu}+\frac{1}{2} \nabla_{\mu} \varphi^{\mu \rho} \nabla^{\nu} \varphi_{\nu \rho}-\frac{1}{2} \nabla_{\mu} \varphi_{\rho}^{\rho} \nabla_{\nu} \varphi^{\mu \nu}+\frac{1}{4} \nabla_{\rho} \varphi_{\mu}^{\mu} \nabla^{\rho} \varphi_{\nu}^{\nu} \\
& +\frac{1}{4}(2-f) \varphi_{\mu \nu} \varphi^{\mu \nu}+\frac{1}{4}(2+f)\left(\phi_{\mu}^{\mu}\right)^{2}+\alpha T_{\mu \nu} \varphi^{\mu \nu} .
\end{aligned}
$$


Here $\alpha$ is some coupling and the tensor $T_{\mu \nu}$ is assumed to be of the form

$$
T_{\mu \nu}=\frac{1}{2} \nabla_{\mu} s \nabla_{\nu} s+\frac{1}{2} \nabla_{\nu} s \nabla_{\mu} s-\frac{1}{2} g_{\mu \nu}\left(\nabla^{\rho} s \nabla_{\rho} s+\frac{1}{2}\left(2 m^{2}-f\right) s s\right) .
$$

The scalar field $s$ has mass squared $m^{2}=\Delta(\Delta-4)$ and $f=k(k+4)$ is the mass squared of the symmetric tensor $\varphi_{\mu \nu}$ transforming in the irrep $[0, k, 0]$ of $\mathrm{SO}(6)$. The massless graviton arises as the particular case $k=0$. For the sake of clarity we suppress the inessential representation index for both the $s^{I}$ and $\varphi_{\mu \nu}^{I}$ fields.

First of all, the Lagrangian (E.1) implies the following equation

$$
\nabla_{\rho} \nabla^{\rho} \phi_{\lambda}^{\lambda}=\nabla_{\rho} \nabla_{\lambda} \phi^{\rho \lambda}-\frac{2-f+d(2+f)}{2-d} \phi_{\lambda}^{\lambda}-\frac{2 \alpha}{2-d} T_{\mu}^{\mu}
$$

where $d$ is the dimension of the AdS space, i.e. $d=5$ for $A d S_{5}$. Using this equation we then find

$$
\begin{aligned}
W_{\mu \nu}^{\rho \lambda} \phi_{\rho \lambda} & \equiv-\nabla_{\rho} \nabla^{\rho} \phi_{\mu \nu}+\nabla_{\mu} \nabla^{\rho} \phi_{\rho \nu}+\nabla_{\nu} \nabla^{\rho} \phi_{\rho \mu}-\nabla_{\mu} \nabla_{\nu} \phi_{\rho}^{\rho}-\left((2-f) \phi_{\mu \nu}+\frac{6+f}{2-d} g_{\mu \nu} \phi_{\lambda}^{\lambda}\right) \\
& =\alpha\left(g_{\mu \rho} g_{\nu \lambda}+g_{\mu \lambda} g_{\nu \rho}+\frac{2}{2-d} g_{\mu \nu} g_{\rho \lambda}\right) T^{\rho \lambda}
\end{aligned}
$$

which defines the modified Ricci operator $W_{\mu \nu}^{\rho \lambda}$ acting on $\phi_{\rho \lambda}$. Applying the derivative $\nabla^{\mu}$ to both sides of this equation we find

$$
f\left(\nabla^{\mu} \phi_{\mu \nu}-\nabla_{\nu} \phi_{\lambda}^{\lambda}\right)=2 \alpha \nabla_{\mu} T_{\nu}^{\mu}
$$

Differentiating this formula, using (E.3), and assuming that $f$ is non-zero we can solve for the trace of $\varphi_{\mu \nu}$ :

$$
\varphi_{\lambda}^{\lambda}=-\frac{\alpha}{2 f(f+3)}\left(3 \nabla_{\mu} \nabla_{\nu} T^{\mu \nu}+f T_{\mu}^{\mu}\right)
$$

By definition, the $z$-integral describing the exchange of a massive symmetric tensor is given by

$$
A_{\mu \nu}\left(w, x_{3}, x_{4}\right)=\int \frac{\mathrm{d}^{d} z \mathrm{~d} z_{0}}{z_{0}^{d+1}} G_{\mu \nu, \lambda \rho}(w, z) T^{\lambda \rho}\left(z, x_{3}, x_{4}\right)
$$

Here the bitensor $G_{\mu \nu, \rho \lambda}(w, z)$ is the bulk-to-bulk propagator for the massive symmetric tensor $\varphi_{\mu \nu}$. It depends on the AdS invariant distance and obeys the equation

$$
W_{\mu \nu}^{\rho \lambda} G_{\rho \lambda, \mu^{\prime} \nu^{\prime}}(w, z)=\left(g_{\mu \mu^{\prime}} g_{\nu \nu^{\prime}}+g_{\mu \nu^{\prime}} g_{\nu \mu^{\prime}}+\frac{2}{2-d} g_{\mu \nu} g_{\mu^{\prime} \nu^{\prime}}\right) \delta(z, w) .
$$

The tensor $T^{\lambda \rho}\left(z, x_{3}, x_{4}\right)$, suppressing the argument $z$, is given by

$$
\begin{aligned}
T_{\mu \nu}\left(x_{3}, x_{4}\right)= & \frac{1}{2} \nabla_{\mu} K_{\Delta}\left(x_{3}\right) \nabla_{\nu} K_{\Delta}\left(x_{4}\right)+\frac{1}{2} \nabla_{\nu} K_{\Delta}\left(x_{3}\right) \nabla_{\mu} K_{\Delta}\left(x_{4}\right) \\
& -\frac{1}{2} g_{\mu \nu}\left(\nabla^{\rho} K_{\Delta}\left(x_{3}\right) \nabla_{\rho} K_{\Delta}\left(x_{4}\right)+\frac{1}{2}\left(2 m^{2}-f\right) K_{\Delta}\left(x_{3}\right) K_{\Delta}\left(x_{4}\right)\right),
\end{aligned}
$$


where the functions $K_{\Delta}(z, x)$ were defined in (D.2).

Following [36], we use $A_{\mu \nu}\left(w, x_{3}, x_{4}\right)=A_{\mu \nu}\left(w-x_{3}, 0, x_{43}\right)$ and then perform a conformal inversion on the integral (E.6) to obtain

$$
A_{\mu \nu}(w, 0, x)=\frac{1}{x^{2 \Delta} w^{4}} J_{\mu \lambda}(w) J_{\nu \rho}(w) I_{\lambda \rho}\left(w^{\prime}-x^{\prime}\right), \quad w^{\prime}=\frac{w_{\mu}}{w^{2}}, \quad x^{\prime}=\frac{x_{\mu}}{x^{2}},
$$

where $J_{\mu \nu}(w)=\delta_{\mu \nu}-2 \frac{w_{\mu} w_{\nu}}{w^{2}}$. We then write down the following ansatz for the tensor $I_{\mu \nu}$

$$
I_{\mu \nu}(w)=g_{\mu \nu} h(t)+P_{\mu} P_{\nu} \phi(t)+\nabla_{\mu} \nabla_{\nu} X(t)+\nabla_{(\mu}\left(P_{\nu)} Y(t)\right) .
$$

Here $t=w_{0}^{2} / w^{2}, g_{\mu \nu}=\delta_{\mu \nu} / w_{0}^{2}, P_{\mu}=\delta_{0 \mu} / w_{0}$, and $h(t), \phi(t), X(t), Y(t)$ are four unknown functions. To find them one has to work out the action of the modified Ricci operator on eq. (E.10). For the individual terms in (E.10) we obtain the following formulae

$$
\begin{aligned}
W_{\mu \nu}^{\rho \lambda}\left[g_{\rho \lambda} h(t)\right]= & \left(4 t^{2}(t-1) h^{\prime \prime}+4 t(t+1) h^{\prime}+\frac{8}{3}(f+3) h\right) g_{\mu \nu}-3 \nabla_{\mu} \nabla_{\nu} h, \\
W_{\mu \nu}^{\rho \lambda}\left[P_{\rho} P_{\lambda} \phi(t)\right]= & g_{\mu \nu}\left(4 t(t-1) \phi^{\prime}+\frac{1}{3}(f+24) \phi\right)-\nabla_{\mu} \nabla_{\nu} \phi \\
& +P_{\mu} P_{\nu}\left(4 t^{2}(1-t) \phi^{\prime \prime}-8 t^{2} \phi^{\prime}+f \phi\right)+\frac{\delta_{0(\mu} w_{\nu)} w_{0}}{\left(w^{2}\right)^{2}}\left(4 t(t-1) \phi^{\prime \prime}+8 t \phi^{\prime}\right) .
\end{aligned}
$$

and

$$
\begin{aligned}
W_{\mu \nu}^{\rho \lambda}\left[\nabla_{\rho} \nabla_{\lambda} X(t)\right]= & g_{\mu \nu}\left(-\frac{4}{3} t^{2}(t-1) X^{\prime \prime}-\frac{4}{3} t(t+1) X^{\prime}\right) f+f \nabla_{\mu} \nabla_{\nu} X, \\
W_{\mu \nu}^{\rho \lambda}\left[\nabla_{\rho}\left(P_{\lambda} Y\right)+\nabla_{\lambda}\left(P_{\rho} Y\right)\right]= & g_{\mu \nu}\left(-\frac{14}{3} Y-\frac{4}{3} t(t-1) Y^{\prime}\right) f \\
& +P_{\mu} P_{\nu}\left(2 Y+4 t Y^{\prime}\right) f+\frac{\delta_{0(\mu} w_{\nu} w_{0}}{\left(w^{2}\right)^{2}}\left(-2 Y^{\prime} f\right) .
\end{aligned}
$$

The basic equation allowing us to determine the unknown functions in (E.10) is derived by using the fundamental equation (E.7):

$$
W_{\mu \nu}^{\rho \lambda}\left[I_{\rho \lambda}(w)\right]=\frac{1}{3} g_{\mu \nu}\left(2 m^{2}-f\right) t^{\Delta}+P_{\mu} P_{\nu}\left(2 \Delta^{2} t^{\Delta}\right)-2 \Delta^{2} t^{\Delta-1} \frac{\delta_{0\{\mu} w_{\nu\}} w_{0}}{\left(w^{2}\right)^{2}} .
$$

To solve this equation we first equate the terms involving $\nabla_{\mu} \nabla_{\nu}$ on both sides:

$$
\nabla_{\mu} \nabla_{\nu}(-3 h-\phi+f X)=0
$$

and pick up the trivial solution

$$
h(t)=-\frac{1}{3} \phi+\frac{f}{3} X .
$$

Now we equate the coefficients of the expression $\frac{\delta_{0(\mu} w_{\nu)} w_{0}}{\left(w^{2}\right)^{2}}$ and find

$$
4 t(t-1) \phi^{\prime \prime}+8 t \phi^{\prime}-2 f Y^{\prime}=-2 \Delta^{2} t^{\Delta-1} .
$$

This equation is trivially integrated to give

$$
Y(t)=a+\frac{1}{2 f}\left(4 t(t-1) \phi^{\prime}+4 \phi+2 \Delta t^{\Delta}\right),
$$


where $a$ is an integration constant. Equating the coefficients of $P_{\mu} P_{\nu}$ we get

$$
4 t^{2}(1-t) \phi^{\prime \prime}-8 t^{2} \phi^{\prime}+f \phi+2 Y f+4 t f Y^{\prime}=2 \Delta^{2} t^{\Delta} .
$$

Substituting here $(\mathbb{E . 1 3})$ we find a closed equation for $\phi$ :

$$
4 t^{2}(t-1) \phi^{\prime \prime}+4 t(3 t-1) \phi^{\prime}+(f+4) \phi+2 a f+2 \Delta(\Delta+1) t^{\Delta}=0 .
$$

Finally we equate the coefficients of $g_{\mu \nu}$ and substitute their (E.12) and (E.13). The resulting equation is used to find $X$ :

$$
\begin{gathered}
X(t)=\frac{1}{8 f(f+3)}\left[12 t^{2}(2 t-1)(t-1) \phi^{\prime \prime}+12 t\left(4 t^{2}+t-3\right) \phi^{\prime}+(36+5 f) \phi\right. \\
\left.+42 a f-3 t^{\Delta}(f+2 \Delta(\Delta-2 \Delta t-3))\right] .
\end{gathered}
$$

Thus, solving eqs.(E.12), (E.13), (E.15) and $(\mathbb{E} .16)$ we can establish the $z$-integral corresponding to a massive spin 2 tensor exchange.

Now we solve equation (E.15) for $\Delta=3, f=12$. The solution regular at both $t=1$ and $t=0$ has the form

$$
\phi(t)=-\frac{3}{2} a-\frac{3}{4} t^{2}, \quad h(t)=\frac{3}{2} a+\frac{3}{20} t^{2}, \quad X(t)=\frac{1}{4} a-\frac{1}{40} t^{2}, \quad Y(t)=\frac{3}{4} a+\frac{1}{8} t^{2} .
$$

It is easy to see that upon substituting the coefficient functions found into the ansatz (E.10), all the terms proportional to the arbitrary integration constant $a$ cancel out. Combining all the pieces together we obtain

$$
I_{\mu \nu}(w)=\frac{3}{20} t^{2} g_{\mu \nu}-\frac{3}{4} t^{2} P_{\mu} P_{\nu}-\frac{1}{40} \nabla_{\mu} \nabla_{\nu} t^{2}+\frac{1}{8} \nabla_{(\mu}\left(P_{\nu)} t^{2}\right) .
$$

Working out the action of the derivatives this formula can be written in the form

$$
I_{\mu \nu}(w)=-\frac{3}{5} t^{2} \frac{w_{\mu} w_{\nu}}{\left(w^{2}\right)^{2}}
$$

This is an amazingly simple expression, even simpler than the corresponding result for the graviton exchange.

To restore the $z$-integral from $I_{\mu \nu}$ one has to let

$$
t=\frac{w_{0}^{\prime 2}}{\left(w^{\prime}-x^{\prime}\right)^{2}} \rightarrow q=x_{34}^{2} \frac{w_{0}}{w_{0}^{2}+\left(\vec{w}-x_{3}\right)^{2}} \frac{w_{0}}{w_{0}^{2}+\left(\vec{w}-x_{4}\right)^{2}},
$$

and also

$$
\frac{1}{w^{2}\left(w^{\prime}-x^{\prime}\right)^{2}} J_{\mu \nu}(w)\left(w^{\prime}-x^{\prime}\right)_{\nu} \rightarrow Q_{\mu}=\frac{\left(x_{3}-w\right)_{\mu}}{\left(x_{3}-w\right)^{2}}-\frac{\left(x_{4}-w\right)_{\mu}}{\left(x_{4}-w\right)^{2}}
$$

In this way we find the following result for the $z$-integral describing the exchange of the spin 2 tensor of $m^{2}=12$ :

$$
A_{\mu \nu}\left(w, x_{3}, x_{4}\right)=-\frac{3}{5 x_{34}^{2}} Q_{\mu} Q_{\nu} K_{2}\left(w, x_{3}\right) K_{2}\left(w, x_{4}\right)
$$


The result (E.21) is symmetric under the exchange $x_{3} \leftrightarrow x_{4}$, as it should be. Owing to the conformal property of $Q_{\mu}$ one can also see that $A_{\mu \nu}$ indeed transforms as a tensor under inversions.

Finally, taking the trace of $(\underline{\mathrm{E} .21})$ and using $Q_{\mu} Q^{\mu}=\frac{x_{34}^{2} w_{0}^{2}}{\left(w-x_{3}\right)^{2}\left(w-x_{4}\right)^{2}}$ (the contraction involves the AdS metric), we get

$$
\phi_{\lambda}^{\lambda}=A_{\lambda}^{\lambda}\left(w, x_{3}, x_{4}\right)=-\frac{3}{5} K_{3}\left(w, x_{3}\right) K_{3}\left(w, x_{4}\right) .
$$

On the other hand, this formula should coincide with (E.5) for $\alpha=1$. Using the formula (E.2) one can show that eq. (E.5) indeed coincides with eq.(E.5). This provides a consistency check on our calculation.

The computation of the remaining $w$-integral does not present any difficulty and is based on the general formula

$$
\nabla_{w}^{\mu} K_{\Delta}\left(w, x_{1}\right) Q_{\mu}=\Delta K_{\Delta+1}\left(w, x_{1}\right)\left[x_{14}^{2} \frac{w_{0}}{\left(w-x_{4}\right)^{2}}-x_{13}^{2} \frac{w_{0}}{\left(w-x_{3}\right)^{2}}\right] .
$$

\section{F Results for Short Multiplet Expansions}

Using the variables $z, x$ defined by

$$
s=z x, \quad t=(1-z)(1-x),
$$

the functions $H_{\mathcal{J}}$, defined in (6.32), can be expressed as

$$
H_{\mathcal{J}}(s, t)=\frac{1}{z-x}\left(z g_{\mathcal{J}}(z)-\frac{1}{2} s g_{\mathcal{J}}(z)+s f_{\mathcal{J}}(z)-(z \leftrightarrow x)\right)
$$

where, from the results in [26], $g_{\mathcal{J}}$ represents contributions corresponding to just twist 0 and $f_{\mathcal{J}}$ to twist 2 . We find

$$
\begin{array}{rlrl}
g_{[2,2,2]}(z)= & 0, & f_{[2,2,2]}(z) & =\frac{10}{9}\left(z^{3}+\frac{z^{3}}{(1-z)^{3}}\right), \\
g_{[3,0,3]}(z)= & 0, & f_{[3,0,3]}(z)=-2\left(\frac{2}{z}-1\right)\left(z^{3}+\frac{z^{3}}{(1-z)^{3}}\right), \\
g_{[1,2,1]}(z)= & -\left(z^{3}+\frac{z^{3}}{(1-z)^{3}}\right), & f_{[1,2,1]}(z)= & \frac{3}{2}\left(z^{3}-\frac{z^{3}}{(1-z)^{3}}\right), \\
g_{[2,0,2]}(z)= & \frac{5}{3}\left(\frac{2}{z}-1\right)\left(z^{3}+\frac{z^{3}}{(1-z)^{3}}\right), & f_{[2,0,2]}(z)=-\frac{5}{2}\left(\frac{2}{z}-1\right)\left(z^{3}-\frac{z^{3}}{(1-z)^{3}}\right), \\
g_{[0,2,0]}(z)= & -\frac{35}{8}\left(z^{3}-\frac{z^{3}}{\left.(1-z)^{3}\right),}\right. & f_{[0,2,0]}(z)=\frac{45}{16}\left(z^{3}+\frac{z^{3}}{(1-z)^{3}}\right), \\
g_{[1,0,1]}(z)= & \frac{35}{6}\left(\frac{2}{z}-1\right)\left(z^{3}-\frac{z^{3}}{(1-z)^{3}}\right), & f_{[1,0,1]}(z)=-\frac{15}{4}\left(\frac{2}{z}-1\right)\left(z^{3}+\frac{z^{3}}{(1-z)^{3}}\right), \\
g_{[0,0,0]}(z)= & -50+\frac{25}{2}\left(z^{3}-\frac{z^{3}}{(1-z)^{3}}\right)+\frac{45}{2}\left(\frac{2}{z}-1\right)\left(z^{3}+\frac{z^{3}}{(1-z)^{3}}\right), \\
& f_{[0,0,0]}(z)=-\frac{15}{4}\left(z^{3}+\frac{z^{3}}{(1-z)^{3}}\right)-\frac{35}{4}\left(\frac{2}{z}-1\right)\left(z^{3}-\frac{z^{3}}{(1-z)^{3}}\right) .
\end{array}
$$


The expansion coefficients for the individual contributions of short representations are given by

$$
\begin{aligned}
& d_{1, \ell}^{[1,4,1]}=2^{\ell-2} \frac{(\ell+2) !(\ell+4) !}{(2 \ell+3) !}(\ell+1), \quad d_{2, \ell}^{[1,4,1]}=2^{\ell-2} \frac{3(\ell+3) !(\ell+5) !}{5(2 \ell+5) !}(\ell+2), \\
& d_{3, \ell}^{[1,4,1]}=2^{\ell-3} \frac{3(\ell+4) !(\ell+5) !}{35(2 \ell+7) !}(\ell+2)(\ell+7), \\
& d_{1, \ell}^{[0,4,0]}=2^{\ell-1} \frac{(\ell+2) !(\ell+3) !}{15(2 \ell+3) !}\left(4 \ell^{2}+20 \ell+51\right), \quad d_{2, \ell}^{[0,4,0]}=2^{\ell-4} \frac{7(\ell+3) !(\ell+4) !}{5(2 \ell+5) !}\left(\ell^{2}+7 \ell+18\right), \\
& d_{3, \ell}^{[0,4,0]}=2^{\ell-5} \frac{9(\ell+4) !(\ell+6) !}{25(2 \ell+7) !}(\ell+3), \quad d_{4, \ell}^{[0,4,0]}=2^{\ell-2} \frac{(\ell+5) !(\ell+6) !}{315(2 \ell+9) !}(\ell+3)(\ell+8), \\
& d_{1, \ell}^{[2,2,2]}=2^{\ell-2} \frac{(\ell+2) !(\ell+4) !}{(2 \ell+3) !}(\ell+1), \quad d_{2, \ell}^{[2,2,2]}=2^{\ell-3} \frac{7(\ell+3) !(\ell+5) !}{9(2 \ell+5) !}(\ell+2), \\
& d_{3, \ell}^{[2,2,2]}=2^{\ell-4} \frac{5(\ell+4) !(\ell+6) !}{21(2 \ell+7) !}(\ell+3), \\
& d_{1, \ell}^{[3,0,3]}=2^{\ell-2} \frac{(\ell+2) !(\ell+4) !}{(2 \ell+3) !}(\ell+1), \quad d_{2, \ell}^{[3,0,3]}=2^{\ell-3} \frac{3(\ell+3) !(\ell+5) !}{5(2 \ell+5) !}(\ell+2), \\
& d_{3, \ell}^{[3,0,3]}=2^{\ell-4} \frac{9(\ell+4) !(\ell+6) !}{35(2 \ell+7) !}(\ell+3), \\
& d_{1, \ell}^{[1,2,1]}=2^{\ell-4} \frac{9(\ell+2) !(\ell+3) !}{5(2 \ell+3) !}\left(\ell^{2}+5 \ell+12\right), \quad d_{2, \ell}^{[1,2,1]}=2^{\ell-2} \frac{(\ell+3) !(\ell+4) !}{35(2 \ell+5) !}\left(8 \ell^{2}+56 \ell+129\right), \\
& d_{3, \ell}^{[1,2,1]}=2^{\ell-5} \frac{9(\ell+4) !(\ell+5) !}{175(2 \ell+7) !}\left(7 \ell^{2}+63 \ell+162\right), \quad d_{4, \ell}^{[1,2,1]}=2^{\ell-3} \frac{(\ell+5) !(\ell+7) !}{147(2 \ell+9) !}(\ell+4), \\
& d_{1, \ell}^{[2,0,2]}=2^{\ell-4} \frac{5(\ell+2) !(\ell+3) !}{3(2 \ell+3) !}\left(\ell^{2}+5 \ell+12\right), \quad d_{2, \ell}^{[2,0,2]}=2^{\ell-4} \frac{(\ell+3) !(\ell+4) !}{7(2 \ell+5) !}\left(5 \ell^{2}+35 \ell+78\right), \\
& d_{3, \ell}^{[2,0,2]}=2^{\ell-3} \frac{3(\ell+4) !(\ell+5) !}{35(2 \ell+7) !}\left(\ell^{2}+9 \ell+23\right), \quad d_{4, \ell}^{[2,0,2]}=2^{\ell-3} \frac{(\ell+5) !(\ell+7) !}{147(2 \ell+9) !}(\ell+4), \\
& d_{1, \ell}^{[0,2,0]}=2^{\ell-7} \frac{27(\ell+2) !(\ell+4) !}{5(2 \ell+3) !}(\ell+1), \quad d_{2, \ell}^{[0,2,0]}=2^{\ell-7} \frac{3(\ell+3) !(\ell+4) !}{5(2 \ell+5) !}\left(7 \ell^{2}+49 \ell+106\right), \\
& d_{3, \ell}^{[0,2,0]}=2^{\ell-7} \frac{3(\ell+4) !(\ell+5) !}{175(2 \ell+7) !}\left(59 \ell^{2}+531 \ell+1552\right), d_{4, \ell}^{[0,2,0]}=2^{\ell-6} \frac{(\ell+5) !(\ell+6) !}{105(2 \ell+9) !}\left(7 \ell^{2}+77 \ell+268\right), \\
& d_{1, \ell}^{[1,0,1]}=2^{\ell-5} \frac{(\ell+2) !(\ell+4) !}{(2 \ell+3) !}(\ell+1), \quad d_{2, \ell}^{[1,0,1]}=2^{\ell-3} \frac{(\ell+3) !(\ell+4) !}{5(2 \ell+5) !}\left(\ell^{2}+7 \ell+15\right), \\
& d_{3, \ell}^{[1,0,1]}=2^{\ell-6} \frac{3(\ell+4) !(\ell+5) !}{35(2 \ell+7) !}\left(5 \ell^{2}+45 \ell+118\right), \quad d_{4, \ell}^{[1,0,1]}=2^{\ell-4} \frac{(\ell+5) !(\ell+6) !}{63(2 \ell+9) !}\left(\ell^{2}+11 \ell+36\right), \\
& d_{2, \ell}^{[0,0,0]}=2^{\ell-5} \frac{3(\ell+3) !(\ell+5) !}{7(2 \ell+5) !}(\ell+2), \quad d_{3, \ell}^{[0,0,0]}=2^{\ell-3} \frac{9(\ell+4) !(\ell+6) !}{35(2 \ell+7) !}(\ell+3), \\
& d_{4, \ell}^{[0,0,0]}=2^{\ell-3} \frac{(\ell+5) !(\ell+7) !}{147(2 \ell+9) !}(\ell+4) .
\end{aligned}
$$

For $\ell=0,1$ the general formulae are no longer correct in all cases, for those cases which are different from what would be given from (F.4) we have

$$
d_{1,0}^{[0,4,0]}=2, \quad d_{2,0}^{[0,4,0]}=\frac{9}{5}, \quad d_{3,0}^{[0,4,0]}=\frac{11}{100}, \quad d_{4,0}^{[0,4,0]}=\frac{2}{441},
$$




$$
\begin{array}{llll}
d_{1,1}^{[1,2,1]}=\frac{162}{35}, & d_{2,1}^{[1,2,1]}=\frac{1912}{1225}, & d_{1,1}^{[1,0,1]}=\frac{3}{5}, & d_{2,1}^{[1,0,1]}=\frac{64}{105}, \\
d_{1,0}^{[2,0,2]}=2, & d_{2,0}^{[2,0,2]}=\frac{9}{14}, & d_{3,0}^{[2,0,2]}=\frac{32}{245}, & d_{2,0}^{[2,2,2]}=\frac{109}{105} \\
d_{1,0}^{[0,2,0]}=0, & d_{1,2}^{[0,2,0]}=\frac{33}{20}, & d_{2,0}^{[0,2,0]}=\frac{45}{112}, & d_{3,0}^{[0,2,0]}=\frac{1829}{19600} \\
d_{2,0}^{[0,0,0]}=\frac{9}{70}, & d_{3,0}^{[0,0,0]}=\frac{61}{1960} . &
\end{array}
$$

\section{References}

[1] J. Maldacena, "The large $N$ limit of superconformal field theories and supergravity", Adv. Theor. Math. Phys. 2 (1998) 231.

[2] G.G. Gubser, I.R. Klebanov and A.M. Polyakov, "Gauge theory correlators from noncritical string theory", Phys.Lett. B428 (1998) 105, hep-th/9802109

[3] E. Witten, "Anti-de Sitter space and holography", Adv.Theor.Math.Phys. 2 (1998) 253, hep-th/9802150

[4] G. Arutyunov, B. Eden and E. Sokatchev, "On non-renormalization and OPE in superconformal field theories," Nucl. Phys. B 619 (2001) 359, hep-th/0105254

[5] B. Eden and E. Sokatchev, "On the OPE of $1 / 2$ BPS short operators in N = 4 SCFT(4)," Nucl. Phys. B 618 (2001) 259, hep-th/0106249.

[6] P.J. Heslop and P.S. Howe, "A note on composite operators in N = 4 SYM," Phys. Lett. B 516 (2001) 367, hep-th/0106238

[7] P.J. Heslop and P.S. Howe, "OPEs and 3-point correlators of protected operators in $\mathrm{N}=4$ SYM," Nucl. Phys. B 626 (2002) 265, hep-th/0107212

[8] P.J. Heslop and P.S. Howe, "Four-point functions in N=4 SYM," hep-th/0211252.

[9] K.A. Intriligator, "Bonus symmetries of $\mathrm{N}=4$ super-Yang-Mills correlation functions via AdS duality," Nucl. Phys. B 551 (1999) 575, hep-th/9811047

[10] P.S. Howe, K.S. Stelle and P.K. Townsend, "Superactions," Nucl. Phys. B 191 (1981) 445.

[11] L. Andrianopoli, S. Ferrara, E. Sokatchev and B. Zupnik, "Shortening of primary operators in N-extended SCFT(4) and harmonic-superspace analyticity," Adv. Theor. Math. Phys. 3 (1999) 1149, hep-th/9912007

[12] G.G. Hartwell and P.S. Howe, "(N,p,q) harmonic superspace," Int. J. Mod. Phys. A10 (1995) 3901, hep-th/9412147 Class. Quant. Grav. 12 (1995) 1823.

[13] A. Galperin, E. Ivanov, S. Kalitsyn, V. Ogievetsky and E. Sokatchev, "Unconstrained N=2 Matter, Yang-Mills And Supergravity Theories In Harmonic Superspace," Class. Quant. Grav. 1 (1984) 469.

A.S. Galperin, E.A. Ivanov, V.I. Ogievetsky and E.S. Sokatchev, "Harmonic Superspace," Cambridge Univ. Press (2001) 306 p.

[14] B. Eden, P.S. Howe and P.C. West, "Nilpotent invariants in N=4 SYM", Phys. Lett. B463 (1999) 19-26, hep-th/9905085

[15] P.S. Howe, C. Schubert, E. Sokatchev and P.C. West, "Explicit construction of nilpotent covariants in N = 4 SYM," Nucl. Phys. B 571 (2000) 71, hep-th/9910011.

[16] P. S. Howe and P. C. West, "AdS/SCFT in superspace," Class. Quant. Grav. 18 (2001) 3143, hep-th/0105218. 
[17] A. Galperin, E.A. Ivanov, V. Ogievetsky and E. Sokatchev, "Harmonic Supergraphs. Green Functions," Class. Quant. Grav. 2 (1985) 601.

[18] B. Eden, P.S. Howe, C. Schubert, E. Sokatchev and P.C. West, "Four-point functions in N=4 supersymmetric Yang-Mills theory at two loops," Nucl. Phys. B557 (1999) 355-379, hep-th/9811172.

[19] B. Eden, A.C. Petkou, C. Schubert and E. Sokatchev, "Partial non-renormalisation of the stress-tensor four-point function in $\mathrm{N}=4 \mathrm{SYM}$ and AdS/CFT," Nucl. Phys. B 607 (2001) 191, hep-th/0009106

[20] B. Eden, P.S. Howe, C. Schubert, E. Sokatchev and P.C. West, "Four-point functions in $\mathrm{N}=4$ supersymmetric Yang-Mills theory at two loops," Nucl. Phys. B 557 (1999) 355, hep-th/9811172 "Simplifications of four-point functions in $\mathrm{N}=4$ supersymmetric Yang-Mills theory at two loops," Phys. Lett. B 466 (1999) 20, hep-th/9906051.

[21] M. Bianchi, S. Kovacs, G. Rossi and Y.S. Stanev, "On the logarithmic behavior in N = 4 SYM theory," JHEP 9908 (1999) 020, hep-th/9906188

[22] B. Eden, C. Schubert and E. Sokatchev, "Three-loop four-point correlator in N = 4 SYM," Phys. Lett. B 482 (2000) 309, hep-th/0003096 "Four-point functions of chiral primary operators in $\mathrm{N}=4$ SYM," hep-th/0010005.

[23] M. Bianchi, S. Kovacs, G. Rossi and Y.S. Stanev, "Anomalous dimensions in N $=4$ SYM theory at order g**4," Nucl. Phys. B 584 (2000) 216, hep-th/0003203.

[24] M. Bianchi, M.B. Green, S. Kovacs and G. Rossi, "Instantons in supersymmetric Yang-Mills and D-instantons in IIB superstring theory," JHEP 9808 (1998) 013, hep-th/9807033.

[25] G. Arutyunov and S. Frolov, "Four-point functions of lowest weight CPOs in N $=4$ SYM(4) in supergravity approximation," Phys. Rev. D 62 (2000) 064016; hep-th/0002170.

[26] F.A. Dolan and H. Osborn, "Superconformal symmetry, correlation functions and the operator product expansion," Nucl. Phys. B 629 (2002) 3, hep-th/0112251.

[27] E. D'Hoker, D.Z. Freedman, S.D. Mathur, A. Matusis and L. Rastelli, "Graviton exchange and complete four-point functions in the AdS/CFT correspondence," Nucl. Phys. B 562 (1999) 353, hep-th/9903196

[28] S.M. Lee, S. Minwalla, M. Rangamani and N. Seiberg, "Three-point functions of chiral operators in $\mathrm{D}=4, \mathrm{~N}=4 \mathrm{SYM}$ at large N," Adv. Theor. Math. Phys. 2 (1998) 697, hep-th/9806074

[29] G.E. Arutyunov and S.A. Frolov, "Quadratic action for type IIB supergravity on AdS(5) x S(5)," JHEP 9908 (1999) 024, hep-th/9811106.

[30] G. Arutyunov and S. Frolov, "Some cubic couplings in type IIB supergravity on AdS(5) x S(5) and three-point functions in $\operatorname{SYM}(4)$ at large N," Phys. Rev. D 61 (2000) 064009, hep-th/9907085

[31] S.M. Lee, "AdS(5)/CFT(4) four-point functions of chiral primary operators: Cubic vertices," Nucl. Phys. B 563 (1999) 349, hep-th/9907108.

[32] G. Arutyunov and S. Frolov, "Scalar quartic couplings in type IIB supergravity on AdS(5) x S(5)," Nucl. Phys. B 579 (2000) 117, hep-th/9912210.

[33] E. D'Hoker, D.Z. Freedman, S.D. Mathur, A. Matusis and L. Rastelli, "Extremal correlators in the AdS/CFT correspondence," hep-th/9908160

[34] G. Arutyunov and S. Frolov, "On the correspondence between gravity fields and CFT operators," JHEP 0004 (2000) 017, hep-th/0003038 
[35] G. Arutyunov and E. Sokatchev, "Implications of superconformal symmetry for interacting $(2,0)$ tensor multiplets," Nucl. Phys. B 635 (2002) 3, hep-th/0201145

[36] E.D'Hoker, D.Z. Freedman and L. Rastelli, "AdS/CFT four-point functions: How to succeed at z-integrals without really trying," Nucl. Phys. B 562 (1999) 395; hep-th/9905049

[37] E.D'Hoker, S.D. Mathur, A. Matusis and L. Rastelli, "The operator product expansion of $\mathrm{N}=4$ SYM and the four-point functions of supergravity," Nucl. Phys. B 589 (2000) 38, hep-th/9911222.

[38] G. Arutyunov, S. Frolov and A.C. Petkou, "Operator product expansion of the lowest weight CPOs in N = 4 SYM(4) at strong coupling," Nucl. Phys. B 586 (2000) 547, hep-th/0005182 "Perturbative and instanton corrections to the OPE of CPOs in N = 4 SYM(4)," Nucl. Phys. B 602 (2001) 238, hep-th/0010137.

[39] G. Arutyunov, B. Eden, A.C. Petkou and E. Sokatchev, "Exceptional non-renormalization properties and OPE analysis of chiral four-point functions in $\mathrm{N}=4 \operatorname{SYM}(4)$," Nucl. Phys. B 620 (2002) 380, hep-th/0103230

[40] L. Hoffmann, L. Mesref and W. Ruhl, "Conformal partial wave analysis of AdS amplitudes for dilaton axion four-point functions," Nucl. Phys. B 608 (2001) 177, hep-th/0012153

[41] F.A. Dolan and H. Osborn, "Conformal four point functions and the operator product expansion," Nucl. Phys. B 599 (2001) 459, hep-th/0011040

[42] F.A. Dolan and H. Osborn, "On short and Semi-Short Representations for Four Dimensional Supersymmetry," hep-th/0209056.

[43] N.I. Usyukina and A.I. Davydychev, "An Approach to the evaluation of three and four point ladder diagrams," Phys. Lett. B 298 (1993) 363. 